\title{
Open-geometry modal method based on transverse electric and transverse magnetic mode expansion for orthogonal curvilinear coordinates
}

Gür, Uur Meriç; Arslanagi, Samel; Mattes, Michael; Gregersen, Niels

Published in:

Physical Review E

Link to article, DOI:

10.1103/PhysRevE.103.033301

Publication date:

2021

Document Version

Peer reviewed version

Link back to DTU Orbit

Citation (APA):

Gür, U. M., Arslanagi, S., Mattes, M., \& Gregersen, N. (2021). Open-geometry modal method based on transverse electric and transverse magnetic mode expansion for orthogonal curvilinear coordinates. Physical Review E, 103(3), [033301]. https://doi.org/10.1103/PhysRevE.103.033301

\section{General rights}

Copyright and moral rights for the publications made accessible in the public portal are retained by the authors and/or other copyright owners and it is a condition of accessing publications that users recognise and abide by the legal requirements associated with these rights.

- Users may download and print one copy of any publication from the public portal for the purpose of private study or research.

- You may not further distribute the material or use it for any profit-making activity or commercial gain

- You may freely distribute the URL identifying the publication in the public portal 


\title{
Open-geometry modal method based on TE and TM mode expansion for orthogonal curvilinear coordinates
}

\author{
Uğur Meriç Gür, Samel Arslanagić, and Michael Mattes \\ Department of Electrical Engineering, Technical University of Denmark, \\ Ørsteds Plads, Building 348, DK-2800 Kongens Lyngby, Denmark \\ Niels Gregersen* \\ Department of Photonics Engineering, Technical University of Denmark, \\ Ørsteds Plads, Building 343, DK-2800 Kongens Lyngby, Denmark
}

(Dated: March 8, 2021)

\begin{abstract}
A novel vectorial modal method is presented based on transverse magnetic (TM) and transverse electric (TE) mode expansion, which significantly simplifies the evaluation of the operator matrix. The method, which features a true open boundary condition, is introduced for an orthogonal curvilinear coordinate system with the specific examples of circular and elliptical geometries presented. We validate the method by considering challenging problems, such as the calculation of spontaneous emission rates, of modal reflection coefficients and of the effect of the emitter spatial misalignment on the spontaneous emission $\beta$ factor. Results are compared with literature.
\end{abstract}

\section{INTRODUCTION}

The optimization of the performance of nanophotonic devices relies on a detailed analysis of relevant figures of merits. Examples of devices of interest include classical light sources such as photonic crystal nanolasers [1] as well as deterministic quantum light sources $[2,3]$ based on photonic nanowires [4-9] or micropillar cavities [10] featuring rotationally symmetric [11] or even elliptical [12] cross-sections for polarization control. The figures of merit being optimized include the spontaneous emission $\beta$ factor [3] describing the ratio of spontaneous emission into the waveguide or cavity mode of interest as well as the modal reflectivity coefficients of the cavity mirrors [4].

Whereas popular optical simulation tools including Finite Difference Time Domain (FDTD) and Finite Element Method (FEM) $[13,14]$ do not allow for direct evaluation of the $\beta$ factor and the reflection coefficients, the family of modal expansion methods [15-28] offers direct insight into the physics by providing full access to the optical mode profiles, propagation constants, and intermodal scattering coefficients. The optical eigenmodes at the heart of the modal method can in some geometries be described semi-analytically [29], however, in general they are determined as series expansions based on appropriate basis functions. In all cases, an appropriate choice of the coordinate system is crucial in ensuring convergence of the solution. The Modal Method (MM) $[15,16]$ was originally developed to study scattering of light by periodic gratings, where the coordinate system is Cartesian, the basis functions are plane waves, and the modes are expanded on the $x$ and $y$ field components of the plane perpendicular to the propagation axis. It was demonstrated that careful treatment $[17,18]$ of the

\footnotetext{
*ngre@fotonik.dtu.dk
}

product of the normal field component to an interface and the permittivity allowed for improved convergence for geometries with large dielectric contrasts, a procedure subsequently summarized in Li's factorization rules [19]. However, for non-rectangular structures, the material boundaries are not aligned with the coordinate axes leading to a zigzag-shaped approximation [20] of the boundaries, and as a result, poor convergence. To improve the convergence for gratings with complex geometrical profiles, normal vector methods [21, 22] based on coordinate transformations were introduced. Here, the Cartesian basis is mapped to curvilinear coordinate axes of the structure such that the interfaces are aligned with a coordinate axis, and the factorization rules can be respected.

While the modal method was initially developed for periodic structures, calculations of the $\beta$ factor and the modal reflectivity coefficient rely critically on a proper modeling of the leakage of light into the surroundings. An open geometry boundary condition based on an absorbing perfectly matched layer (PML) formalism implemented in the form of a complex coordinate transformation was initially suggested [23]. At the same time, a Fourier-Bessel basis appropriate for the rotationally symmetric coordinate system was proposed [24]. In this work, a true open geometry boundary condition based on an infinite domain combined with an equidistant discretization of the continuous $k$-space was implemented. More recently, non-uniform $k$-space discretization schemes for rotationally symmetric [25] and Cartesian coordinates [26] enabling significantly improved convergence were proposed. However, the modeling of state-of-the-art singlephoton sources requires a modal method formalism allowing for orthogonal curvilinear coordinate systems, e.g. elliptical coordinates [12]. While the evaluation of the infinite integrals in Refs. [24-26] arising due to the infinite domain was possible due to the well-known properties of the exponential and Bessel functions, significant mathematical complexity arises when generalizing the formal- 
ism to orthogonal curvilinear systems.

There is thus a need for a modal method allowing for 1) orthogonal curvilinear coordinates and 2) true open geometry boundary conditions, which in addition should 3) be mathematically straightforward to implement. In this work, a full vectorial modal method fulfilling these demands is presented. The main novelty is the expansion on full vectorial transverse electric (TE) and transverse magnetic (TM) basis modes instead of individual $x$ and $y$ field components. We will show that this choice of TE and TM basis modes significantly simplifies the evaluation of the operator matrix elements such that only definite integrals of products of scalar basis functions need to be computed. We benchmark our formalism by presenting simulations of $\beta$ factors and reflection coefficients in rotationally symmetric as well as elliptical geometries, and we compare our results with the literature. The manuscript is organized as follows: Section II presents an overview of the theory of the formulation with details given in the Appendix. In Section III, we present our numerical results and compare them with the literature. In Section IV, we discuss the new formalism, followed by a conclusion in Section V. The Supplementary Information (SI) contains the complete formalism with details of all derivations and can be used as an implementation guide to students at a graduate level.

\section{THEORY}

With the modal method, we aim at solving electromagnetic problems in the frequency domain for structures that are dividable into uniform sections along a propagation axis, usually chosen as the $z$ axis, where each section features an arbitrary cross-section. To illustrate the principle, a simple device having a circular cross-section and consisting of three layers is considered in Fig. 1(a). The different layers have different core radii and refractive indices. All the core layers are surrounded by cladding material usually chosen to be vacuum extending radially to infinity. With the help of the time-harmonic Maxwell equations under $e^{-i \omega t}$ time convention and assuming non-magnetic materials, we have $\nabla \times \mathbf{E}(\mathbf{r})=i \omega \mu_{0} \mathbf{H}(\mathbf{r})$ and $\nabla \times \mathbf{H}(\mathbf{r})=-i \omega \epsilon(\mathbf{r}) \mathbf{E}(\mathbf{r})$, in which $\mathbf{E}(\mathbf{r})$ and $\mathbf{H}(\mathbf{r})$ are the electric and the magnetic fields, respectively, at an arbitrary position $\mathbf{r}, \omega$ being the angular frequency, $\epsilon(\mathbf{r})=\epsilon_{r}(\mathbf{r}) \epsilon_{0}$ and $\mu_{0}$ being the permittivity and permeability, respectively. The wave equation for the electric field is then obtained as

$$
\nabla \times \nabla \times \mathbf{E}(\mathbf{r})=\epsilon_{r} k_{0}^{2} \mathbf{E}(\mathbf{r})
$$

where $k_{0}=\omega / c$ is the free-space wave number. We then consider each layer separately as in Fig. 1(b), assuming uniformity along the propagation axis and we compute eigenmodes which are solutions to (1) for the uniform geometry, employing separation of variables to describe the axial variation using a complex exponential. We then expand the electric and the magnetic fields on the eigenmodes $\mathbf{e}_{j}$ and $\mathbf{h}_{j}, j$ being the eigenmode index, as,

$$
\begin{aligned}
\mathbf{E}\left(\mathbf{r}_{\perp}, z\right)= & \sum_{j} a_{j} \mathbf{e}_{j}\left(\mathbf{r}_{\perp}\right) \exp \left(i \beta_{j} z\right) \\
& +\sum_{j} b_{j} \mathbf{e}_{j}\left(\mathbf{r}_{\perp}\right) \exp \left(-i \beta_{j} z\right), \\
\mathbf{H}\left(\mathbf{r}_{\perp}, z\right)= & \sum_{j} a_{j} \mathbf{h}_{j}\left(\mathbf{r}_{\perp}\right) \exp \left(i \beta_{j} z\right) \\
& +\sum_{j} b_{j} \mathbf{h}_{j}\left(\mathbf{r}_{\perp}\right) \exp \left(-i \beta_{j} z\right),
\end{aligned}
$$

where $a_{j}$ and $b_{j}$ are the modal expansion coefficients, $\beta_{j}$ is the propagation constant, $\mathbf{r}_{\perp}$ represents the transverse coordinates and the summations include both discrete guided and continuous radiation modes. As discussed in detail in SI Sections 4.2-3, a discretization of the continuous set of radiation modes is introduced, and the numerical treatment of the radiation modes is fully equivalent to that of the discrete guided modes. To calculate the eigenmodes, we expand each eigenmode on basis modes as discussed in detail in Section II A, where a novel approach introducing TE and TM basis modes is proposed. Combining Maxwell's equations with these basis mode expansions leads to a linear eigenvalue problem with eigenvectors containing amplitude coefficients and eigenvalues being the squares of the $\beta_{j}$ in Eqs. $(2,3)$. As illustrated in Fig. 1(c), the scattering of light at the interface between two layers $j$ and $j+1$ is then described using interface reflection $\overline{\bar{R}}_{j, j+1}$ and transmission $\overline{\bar{T}}_{j, j+1}$ matrices obtained by matching the transverse fields at the interface. Scattering from multiple layers is computed iteratively from the interface matrices using the $\mathrm{S}$ matrix formalism [27, 28], and for the spontaneous emission calculations the eigenmode expansion coefficients in Eqs. $(2,3)$ are obtained by projecting the imposed current source onto the eigenmodes [28] as discussed further in Section IIIB.

In the next subsections, we give the details of the novel basis modes, their construction procedure for an open boundary problem, and we show how they yield simple coupling integrals that can be solved analytically.

\section{A. Basis Modes}

Defining the basis modes used subsequently in the expansion of the eigenmodes is a crucial step of the proposed method. To define the basis modes, we first define the basis function $\Psi\left(\mathbf{r}_{\perp}\right)$ using the scalar Helmholtz equation for a homogeneous, isotropic, and unbounded medium, usually vacuum,

$$
\nabla^{2} f_{z}(\mathbf{r})+k_{0}^{2} f_{z}(\mathbf{r})=0
$$

where $f_{z}$ represents the axial $z$ component of the electric, $e_{z}$, or magnetic, $h_{z}$, field. The solution is of the form

$$
f_{z}\left(\mathbf{k}_{\perp}, \mathbf{r}\right)=\Psi\left(\mathbf{k}_{\perp}, \mathbf{r}_{\perp}\right) e^{i \beta z}
$$




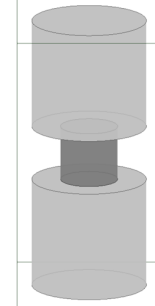

(a)

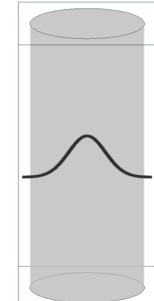

(b)

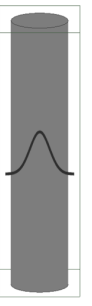

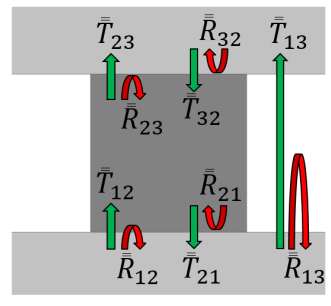

(c)
FIG. 1. (a): An electromagnetic environment consisting of three rotationally symmetric layers. (b): The layers of the geometry assuming uniformity along the propagation axis. (c): The scattering between the layers. $\overline{\bar{R}}_{12}-\overline{\bar{T}}_{12}, \overline{\bar{R}}_{21}-\overline{\bar{T}}_{21}, \overline{\bar{R}}_{23}-\overline{\bar{T}}_{23}$, $\overline{\bar{R}}_{32}-\overline{\bar{T}}_{32}$ are interface reflection and transmission matrices and $\overline{\bar{R}}_{13}-\overline{\bar{T}}_{13}, \overline{\bar{R}}_{31}-\overline{\bar{T}}_{31}$ represent scattering matrices for remote layers computed using $\mathrm{S}$ matrix formalism.

and the basis function $\Psi\left(\mathbf{k}_{\perp}, \mathbf{r}_{\perp}\right)$ fulfills

$$
\nabla_{\perp}^{2} \Psi\left(\mathbf{k}_{\perp}, \mathbf{r}_{\perp}\right)+k_{0}^{2} \Psi\left(\mathbf{k}_{\perp}, \mathbf{r}_{\perp}\right)=\beta^{2} \Psi\left(\mathbf{k}_{\perp}, \mathbf{r}_{\perp}\right),
$$

where $k_{\perp}=\left|\mathbf{k}_{\perp}\right|=\sqrt{k_{0}^{2}-\beta^{2}}$ is the transverse wavenumber. The existence of an analytical solution to (6) is discussed further in Section IV. For now, we assume that the basis function $\Psi\left(\mathbf{k}_{\perp}, \mathbf{r}_{\perp}\right)$ defined by (6) can be easily evaluated, and we expand the axial field components on this set of basis functions. Depending on the coordinate system, separable basis functions of the form $\Psi_{\mathrm{TX}}\left(k_{\perp}, m, \mathbf{r}_{\perp}\right)=U_{\mathrm{TX}}\left(k_{\perp}, m, u\right) V_{\mathrm{TX}}\left(k_{\perp}, m, v\right)$ may be available, where $u$ and $v$ are the transverse generalized orthogonal coordinates, $m$ is the angular order and TX $=$ TM, TE, where the TX subindex labels the relevant subset of basis functions used to form the basis modes from orthogonality considerations as discussed further in Appendix A. In this work, we consider geometries with $\epsilon_{r}(u)$ independent of $v$, and where $\Psi_{\mathrm{TX}}$ is a periodic function in $v$ with period $2 \pi$ such that $\Psi_{\mathrm{TX}}(u, v)=\Psi_{\mathrm{TX}}(u, v+2 \pi)$. For the coordinate systems considered here (rotationally symmetric, elliptical), the basis functions are separable, and $U$ and $V$ become radial and angular basis functions, respectively. The basis function should be chosen such that it fulfills the orthonormalization condition

$$
\begin{gathered}
\int_{v=0}^{v=2 \pi} \int_{u=0}^{u=\infty} \Psi\left(k_{\perp}, m, u, v\right) \Psi\left(k_{\perp}^{\prime}, m^{\prime}, u, v\right) l_{u} l_{v} d u d v \\
=\delta\left(k_{\perp}-k_{\perp}^{\prime}\right) \delta_{m m^{\prime}}
\end{gathered}
$$

where $l_{u, v}$ are geometrical scaling factors [30] of the specific coordinate system and the $u$ integration is to infinity as we are considering an open (infinite) lateral geometry. In the following expressions, the $k_{\perp}$ index represents the continuous transverse wavenumber including the angular orders $m$. Consequently, we omit using $m$ in the indexing for a simpler notation unless it is of higher importance. Our formalism can also be applied to a closed or periodic geometry, where the upper bound of the $u$ integration then becomes a finite number, the first term on the RHS of (7) becomes a Kronecker delta replacing the Dirac delta distribution, and the boundary conditions lead to a discretization of the allowed in-plane wavenumbers, $k_{\perp, q}$.

To define the basis modes we now consider the vacuum case $\epsilon_{r}(\mathbf{r})=1$, introduce the scaling $\mathbf{H}^{\prime}=\mathbf{H} \omega \mu_{0}$ and assume axial fields $e_{z}\left(k_{\perp}, \mathbf{r}\right)$ and $h_{z}^{\prime}\left(k_{\perp}, \mathbf{r}\right)$ of the form of (5). We then expand Maxwell's equations in orthogonal curvilinear coordinates using Eq. (B1) leading to the expressions for the transverse components of the electric, $e_{u, v}$, and magnetic, $h_{u, v}^{\prime}$, fields in terms of the axial components $e_{z}$ and $h_{z}^{\prime}$ given by

$$
\begin{aligned}
& e_{u}\left(k_{\perp}, u, v\right)=i \frac{\beta}{k_{\perp}^{2} l_{u}} \frac{\partial e_{z}}{\partial u}+\frac{i}{k_{\perp}^{2} l_{v}} \frac{\partial h_{z}^{\prime}}{\partial v}, \\
& e_{v}\left(k_{\perp}, u, v\right)=i \frac{\beta}{k_{\perp}^{2} l_{v}} \frac{\partial e_{z}}{\partial v}-\frac{i}{k_{\perp}^{2} l_{u}} \frac{\partial h_{z}^{\prime}}{\partial u}, \\
& h_{u}^{\prime}\left(k_{\perp}, u, v\right)=-i \frac{k_{0}^{2}}{k_{\perp}^{2} l_{v}} \frac{\partial e_{z}}{\partial v}+i \frac{\beta}{k_{\perp}^{2} l_{u}} \frac{\partial h_{z}^{\prime}}{\partial u}, \\
& h_{v}^{\prime}\left(k_{\perp}, u, v\right)=i \frac{k_{0}^{2}}{k_{\perp}^{2} l_{u}} \frac{\partial e_{z}}{\partial u}+i \frac{\beta}{k_{\perp}^{2} l_{v}} \frac{\partial h_{z}^{\prime}}{\partial v},
\end{aligned}
$$

where we in the following skip the ' notation for the scaled magnetic field for simplicity. Now, by setting either $e_{z}$ or $h_{z}$ to zero, the transverse fields are naturally decomposed into TM and TE field components. Unlike typical modal method implementations where the transverse field expansion is performed on the two individual transverse field components [15-26], we employ an expansion on transverse (TM or TE) basis modes $\mathbf{f}_{\mathrm{TX}}$ and $\mathbf{g}_{\mathrm{TX}}$ such that

$$
\begin{array}{r}
\mathbf{e}_{\perp}(u, v)=\int\left[c_{\mathrm{TM}}^{E_{\perp}}\left(k_{\perp}\right) \mathbf{f}_{\mathrm{TM}}\left(k_{\perp}, u, v\right)\right. \\
\left.+c_{\mathrm{TE}}^{E_{\perp}}\left(k_{\perp}\right) \mathbf{g}_{\mathrm{TE}}\left(k_{\perp}, u, v\right)\right] d k_{\perp}, \\
\mathbf{h}_{\perp}(u, v)=\int\left[c_{\mathrm{TM}}^{H_{\perp}}\left(k_{\perp}\right) \mathbf{g}_{\mathrm{TM}}\left(k_{\perp}, u, v\right)\right. \\
\left.+c_{\mathrm{TE}}^{H_{\perp}}\left(k_{\perp}\right) \mathbf{f}_{\mathrm{TE}}\left(k_{\perp}, u, v\right)\right] d k_{\perp},
\end{array}
$$

where $c_{\mathrm{TX}}^{E_{\perp}, H_{\perp}}$ are the expansion coefficients for the inplane $u, v$ field components and $\mathbf{f}_{\mathrm{TX}}$ and $\mathbf{g}_{\mathrm{TX}}$ are the TM or TE basis modes obtained from the basis function $\Psi_{\mathrm{TX}}\left(k_{\perp}, u, v\right)$ as

$$
\begin{aligned}
f_{\mathrm{TX}, u}\left(k_{\perp}, \mathbf{r}_{\perp}\right) & =\frac{1}{k_{\perp} l_{u}} \frac{\partial}{\partial u} \Psi_{\mathrm{TX}}\left(k_{\perp}, u, v\right), \\
f_{\mathrm{TX}, v}\left(k_{\perp}, \mathbf{r}_{\perp}\right) & =\frac{1}{k_{\perp} l_{v}} \frac{\partial}{\partial v} \Psi_{\mathrm{TX}}\left(k_{\perp}, u, v\right), \\
g_{\mathrm{TM}, u}\left(k_{\perp}, \mathbf{r}_{\perp}\right) & =-f_{\mathrm{TM}, v}\left(k_{\perp}, \mathbf{r}_{\perp}\right), \\
g_{\mathrm{TM}, v}\left(k_{\perp}, \mathbf{r}_{\perp}\right) & =f_{\mathrm{TM}, u}\left(k_{\perp}, \mathbf{r}_{\perp}\right), \\
g_{\mathrm{TE}, u}\left(k_{\perp}, \mathbf{r}_{\perp}\right) & =f_{\mathrm{TE}, v}\left(k_{\perp}, \mathbf{r}_{\perp}\right), \\
g_{\mathrm{TE}, v}\left(k_{\perp}, \mathbf{r}_{\perp}\right) & =-f_{\mathrm{TE}, u}\left(k_{\perp}, \mathbf{r}_{\perp}\right) .
\end{aligned}
$$

Our basis modes are the transverse components of welldefined solutions to the wave equation for a homogeneous, isotropic, and unbounded medium, e.g. vacuum, 
described by the specific choice of orthogonal curvilinear coordinates. This choice of expansion basis leads to simple coupling integrals for the operator matrices as shown in the next subsection. Due to symmetry considerations, we limit the basis modes in the expansions $(12,13)$ to a subset of the total set of basis modes. Examples of subsets for circular and elliptical geometries are given in Appendix A.

\section{B. Eigenmodes of the Inhomogeneous Medium}

We now determine the eigenmodes of the inhomogeneous medium using the basis modes constructed for a homogeneous medium. The open geometry boundary condition leads to a continuous and unbounded in-plane wavenumber distribution, and in a computer environment, a truncation for the upper limit $k_{\max }$ and a discretization $k_{\perp, q}$ of the continuous integration range are required such that

$$
\int_{0}^{\infty} f\left(k_{\perp}\right) d k_{\perp} \rightarrow \int_{0}^{k_{\max }} f\left(k_{\perp}\right) d k_{\perp} \rightarrow \sum_{q=1}^{q=N} f\left(k_{\perp, q}\right) \Delta k_{\perp, q}
$$

where $N$ is the number of $k_{\perp}$ space samples for a single angular order and we allow for a non-uniform sampling of the $k_{\perp}$ space by introducing a non-equidistant $q$-dependent discretization width $\Delta k_{\perp, q}$ in the Riemann sum. Non-uniform discretization schemes [25] can in some cases significantly improve the convergence and are used for the results presented in Section III.

Using the discretization (20), the expansions $(12,13)$ can be inserted into Maxwell's equations in orthogonal curvilinear coordinates, as shown in detail in Appendix $\mathrm{B}$, to obtain a final eigenvalue equation

$$
\overline{\bar{O}}_{\mathrm{eh}} \overline{\bar{O}}_{\mathrm{he}}\left[\begin{array}{c}
\bar{c}_{\mathrm{TM}}^{E_{\perp}} \\
\bar{c}_{\mathrm{TE}}^{E_{\perp}}
\end{array}\right]=\beta^{2}\left[\begin{array}{c}
\bar{c}_{\mathrm{TM}}^{E_{\perp}} \\
\bar{c}_{\mathrm{TE}}^{E_{\perp}}
\end{array}\right]
$$

in which $\overline{\bar{O}}_{\text {eh }}$ and $\overline{\bar{O}}_{\text {he }}$ are $2 N M \times 2 N M$ matrices representing the operators of Maxwell's curl equations expanded on the basis modes, where $M$ is the number of angular orders considered. Each matrix consists of four $N M$ by $N M$ blocks representing the interactions between TM and TE modes, i.e. TM-TM, TM-TE, TETM and TE-TE coupling, given by

$$
\begin{aligned}
& \overline{\bar{O}}_{\text {eh }}=\left[\begin{array}{ll}
\overline{\bar{O}}_{\text {eh }}^{\text {TMTM }} & \overline{\bar{O}}_{\text {eh }}^{\mathrm{TMTE}} \\
\overline{\bar{O}}_{\mathrm{eh}}^{\mathrm{TETM}} & \overline{\bar{O}}_{\mathrm{eh}}^{\mathrm{TETE}}
\end{array}\right]=\left[\begin{array}{cc}
\overline{\bar{O}}_{\mathrm{eh}}^{\mathrm{TMTM}} & 0 \\
0 & \overline{\overline{\mathbf{I}}}_{N M \times N M}
\end{array}\right], \\
& \overline{\bar{O}}_{\mathrm{he}}=\left[\begin{array}{ll}
\overline{\bar{O}}_{\mathrm{he}}^{\mathrm{TMTM}} & \overline{\bar{O}}_{\mathrm{he}}^{\mathrm{TMTE}} \\
\overline{\bar{O}}_{\mathrm{he}}^{\mathrm{TETM}} & \overline{\bar{O}}_{\mathrm{he}}^{\mathrm{TETE}}
\end{array}\right]
\end{aligned}
$$

in which $\overline{\overline{\mathbf{I}}}_{N M \times N M}$ is the $N M$ by $N M$ identity matrix. While including all subsets of basis modes is possible when evaluating the operator matrices $(22,23)$, the symmetries of the coordinate system lead to decoupling of subsets of basis modes belonging to different symmetry classes. It is thus possible to consider one symmetry class at a time in the calculation of the eigenvalue problem (21), and this procedure significantly reduces the computation time. As derived in detail in Appendix B, the TM-TM block of the $\overline{\bar{O}}_{\text {eh }}$ matrix is given by

$$
\begin{aligned}
\overline{\bar{O}}_{\mathrm{eh}, q^{\prime} q}^{\mathrm{TMTM}}= & -k_{\perp, q^{\prime}} k_{\perp, q} \int_{0}^{u_{b}} \int_{0}^{2 \pi} l_{u} l_{v} \Psi_{\mathrm{TM}, q^{\prime}}(u, v) \\
& \times \frac{\Delta \eta_{r}(u)}{k_{0}^{2}} \Psi_{\mathrm{TM}, q}(u, v) d v d u \Delta k_{\perp, q} \\
& +\delta_{q^{\prime} q}\left(1-\frac{\eta_{r, b} k_{\perp, q}^{2}}{k_{0}^{2}}\right),
\end{aligned}
$$

where $\Delta \eta_{r}(u)=\eta_{r}(u)-\eta_{r, b}$ with $\eta_{r}(u)=1 / \epsilon_{r}(u)$ is the relative impermittivity and $\eta_{r, b}$ is the cladding relative impermittivity. The subindex $q$ indexes the $k_{\perp}$ discretization and the short-hand notation $\Psi_{\mathrm{TX}, q}(u, v)=$ $\Psi_{\mathrm{TX}}\left(k_{\perp, q}, u, v\right)$ has been introduced.

Note that $\Delta \eta_{r}(u)=0$ for $u>u_{b}$, where the finite coordinate $u_{b}$ marks the start of the cladding. For a piecewise constant index profile, the coupling integral in the expression (24) can be easily evaluated as shown in Appendix C. For elliptical structures (using the short-hand notations $U_{q}(u)=U\left(k_{\perp, q}, u\right)$ and $\left.V_{q}(v)=V\left(k_{\perp, q}, v\right)\right)$, the relevant integral is given by

$$
\begin{aligned}
& \int_{u=u_{\mathrm{a}}}^{u=u_{\mathrm{b}}} \int_{v=0}^{v=2 \pi} l_{u} l_{v} U_{q}(u) V_{q}(v) U_{q^{\prime}}(u) V_{q^{\prime}}(v) d v d u \\
& =\frac{\left[\left(U_{q}(u) \frac{\partial U_{q^{\prime}}(u)}{\partial u}-U_{q^{\prime}}(u) \frac{\partial U_{q}(u)}{\partial u}\right]_{u_{\mathrm{a}}}^{u_{\mathrm{b}}} \int_{0}^{2 \pi} V_{q}(v) V_{q^{\prime}}(v) d v\right.}{k_{\perp, q}^{2}-k_{\perp, q^{\prime}}^{2}}
\end{aligned}
$$

as derived in Appendix C. We observe that the evaluation of the matrix element (25) requires only the knowledge of the radial basis function $U$ and its derivative $\partial U / \partial u$ at the specific points corresponding to discontinuities in the piece-wise constant permittivity profile. In the general case (C5) when $\Psi(u, v)$ is not separable, only line integrations of $\Psi(u, v)$ at the discontinuity points are needed. This represents a majors asset of our method. As discussed in Appendix B, for the non-magnetic materials considered here the TM-TE and TE-TM blocks of $\overline{\bar{O}}_{\text {eh }}$ are zero, and the TE-TE block is simply the identity matrix. The calculation of the operator matrix $\overline{\bar{O}}_{\text {he }}$ is more extensive as all four blocks are non-zero. As shown in Appendix B, two additional integrals Eqs. (B23,B24) appear. However these again can be computed simply from evaluation of the radial basis function at $\epsilon_{r}$ discontinuity points.

Finally, the linear eigenvalue problem Eq. (21) can be solved numerically and the resulting eigenvectors $\bar{c}^{E_{\perp}}=$ $\left[\bar{c}_{\mathrm{TM}}^{E_{\perp}} \bar{c}_{\mathrm{TE}}^{E_{\perp}}\right]^{T}$ are the expansion coefficients of the basis 


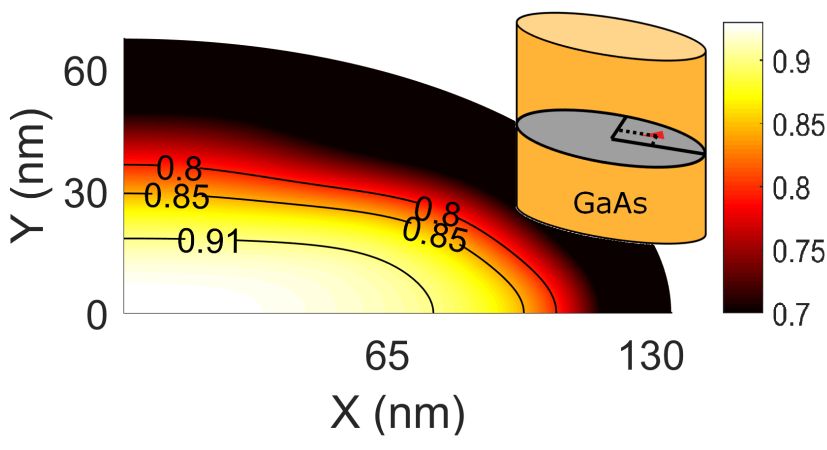

FIG. 2. Intensity map for the spontaneous emission $\beta_{\mathrm{x}}$ factor as a function of emitter location for an elliptical nanowire having a semi-major axis of $135 \mathrm{~nm}$ and a semi-minor axis of $67.5 \mathrm{~nm}$.

modes in Eq. (12) and the eigenvalues $\beta^{2}$ are the corresponding propagation constants squared for each eigenmode. The expansion coefficients for the magnetic field are straightforwardly obtained using the $\overline{\bar{O}}_{\text {he }}$ matrix. The $z$ components of the electric and magnetic fields can be computed from the lateral components as discussed in detail in SI Section 3.3.

\section{PERFORMANCE}

In this section, we demonstrate the reliability and performance of the proposed method by comparing results of several structures with the corresponding ones in the literature. As a first benchmark case, we compute the effect of the emitter location on the spontaneous emission factor, $\beta$, for a quantum emitter inside an elliptical semiconductor nanowire, and we compare the performance of the MM to a commercial FEM solver. Additional benchmarking is presented for the rotationally symmetric nanowire for which we compute spontaneous emission as well as reflection from a nanowire-metal mirror interface. The selected cases are relevant in the context of nanowire single-photon source engineering [3-9] and represent highly challenging problems either not treatable using other numerical methods or requiring orders of magnitude more computational time to tackle.

\section{A. Benchmark I: Spontaneous Emission in an Elliptical Nanowire}

\section{Emitter Location Study}

We first consider the spontaneous emission from an emitter placed in an elliptical nanowire surrounded by unbounded vacuum. The elliptical cross-section is used in single-photon source engineering to selectively deconfine one of the two otherwise degenerate orthogonally polarized $\mathrm{HE}_{11}$ modes and thus establish polarization control of the spontaneous emission as discussed in detail in Ref.
[9]. Here, the spontaneous emission factor $\beta_{\mathrm{x}}$ describing the emission from a quantum dot without a preferential dipole orientation (in the plane) into the $\mathrm{x}$-polarised $\mathrm{HE}_{11}$ mode is given by

$$
\beta_{\mathrm{x}}=\frac{\Gamma_{1, \mathrm{x}}^{\mathrm{x}}+\Gamma_{1, \mathrm{x}}^{\mathrm{y}}}{\Gamma_{\mathrm{all}}^{\mathrm{x}}+\Gamma_{\mathrm{all}}^{\mathrm{y}}}
$$

where $\Gamma_{j, \mathrm{p}}^{\mathrm{q}}$ is the emission rate into the p-polarized mode $j$ from a q-oriented dipole [9], and $\Gamma_{\text {all }}^{\mathrm{q}}$ is the total emission rate including both guided and radiation modes. Therefore, an accurate calculation of the open-geometry radiation modes, is required. The spontaneous emission rate $\Gamma$ can be calculated by considering a classical system and using the correspondence principle [31], which states that the normalized spontaneous emission rate is equal to normalized power $\Gamma_{j} / \Gamma_{\text {bulk }}=P_{j} / P_{\text {bulk }}$ emitted by a classical dipole, where $\Gamma$ and $P$ represent spontaneous emission rate and power flow into either the $j$ th mode or into the bulk medium, respectively. $P_{\text {bulk }}$ can be evaluated by a closed-form expression [31]. The power coupled to a specific mode $j$ from an external excitation is determined from the relation $P_{j}=-\frac{1}{2} \int \operatorname{Re}\left[\mathbf{J}^{*}(\mathbf{r}) \cdot \mathbf{E}_{j}(\mathbf{r})\right] d \mathbf{r}$, where $\mathbf{J}$ is the source current distribution. For a point dipole, we have $\mathbf{J}(\mathbf{r})=\mathbf{J}_{\mathrm{PD}} \delta\left(\mathbf{r}-\mathbf{r}_{\mathrm{PD}}\right), \mathbf{J}_{\mathrm{PD}}$ and $\mathbf{r}_{\mathrm{PD}}$ being the dipole moment and the location of the dipole, respectively. As discussed in detail in the SI Sections 4.2-3, for properly normalized eigenmodes, the modal expansion coefficients in Eq. (2) are given by $a_{j}=-\mathbf{J}_{\mathrm{PD}} \cdot \mathbf{e}_{j}\left(\mathbf{r}_{\mathrm{PD}}\right) / 2$ [28], and one obtains immediately

$$
P_{j}=\frac{1}{4} \operatorname{Re}\left(\left[\mathbf{J}_{\mathrm{PD}} \cdot \mathbf{e}_{j}\left(\mathbf{r}_{\mathrm{PD}}\right)\right]\left[\mathbf{J}_{\mathrm{PD}}^{*} \cdot \mathbf{e}_{j}\left(\mathbf{r}_{\mathrm{PD}}\right)\right]\right),
$$

with the total power given by

$$
P_{\text {all }}=\sum_{j} P_{j}
$$

In the fabrication process, the position of quantum dot is not always well controlled, and it is thus of interest to understand the impact of spatial misalignment on the spontaneous emission factor $\beta$. Following Ref. [9], we compute the factor $\beta_{\mathrm{x}}$ as a function of the emitter location in an infinite elliptical GaAs $\left(n_{\mathrm{GaAs}}=3.45\right)$ nanowire in vacuum having a semi-major axis of $135 \mathrm{~nm}$ and a semi-minor axis of $67.5 \mathrm{~nm}$, shown in the inset of Fig. 2. The excitation is a point dipole radiating at a wavelength of $\lambda_{0}=950 \mathrm{~nm}$, and Figure 2 presents the factor $\beta_{\mathrm{x}}$ as function of emitter position. Our novel MM, combined with a non-uniform $k$-space sampling scheme as proposed in [25], is used with $k_{\max }=20 k_{0}$. Unlike circular geometries, where different angular orders $m$ are decoupled, for elliptical geometries one must include several angular orders $m$ in the calculations (further discussed in Section III A 2), and we have chosen a total number of angular orders of $M=8$ with a total of 100 $k$-space samples for each angular order (800 in total). 


\section{Convergence Study \& Comparison}

In this section, the modal method is compared with a commercial FEM-based solver to demonstrate the effectiveness of the MM. The quantity under study is the total spontaneous emission rate $\Gamma_{\text {all }}^{\mathrm{x}}$ for the geometry in Fig. 2, which is directly accessible also for the FEM. In this comparative study, the emitter is placed at $\mathrm{X}=\mathrm{Y}=$ 0 , however no symmetries are exploited. An Intel Xeon E5-1660 3.2 GHz processor is used for all the simulations.

For the MM, three convergence parameters are relevant: the total number of angular modes $(M)$, the number of $k$-space discretization points $(N)$, and the truncation $k_{\max }$ of the $k$ space. Their influence on the spontaneous emission rate and the computation time is presented in Figs. 3(a-c). The rate is plotted in Figs. 3(a,b) as function of a single convergence parameter keeping the other parameters constant at the value represented by the blue circles in the neighboring figures. As example, in Fig. 3(a) $M$ is increased from 1 to 16 with constant $N=100$ and $k_{\max }=20 k_{0}$. However, in Fig. 3 (c), both $k_{\max }$ and $N$ are varied as follows: The sampling grid parameters $\delta_{1}$ and $\delta_{2}$ [25] are initially defined for $k_{\max }=20 k_{0}$ and $N=100$ according to the nonequidistant sampling scheme [25]. The spontaneous emission rate for $k_{\max } \neq 20 k_{0}$ is subsequently computed for these values of $\delta_{1}$ and $\delta_{2}$ by subtracting $\left(k_{\max }<20 k_{0}\right)$ or adding $\left(k_{\max }>20 k_{0}\right) k$-space sample points, such that only the number M3 of $k$-space points in Eq. (12) of Ref. $[25]$ is varied.

We present the corresponding convergence analysis for FEM in Figs. 3(e-g). An initial mesh is defined, and a first numerical convergence parameter is the number of mesh refinements. The computational domain is terminated by a radiation boundary condition, and additional convergence parameters are the lateral $L x=L y$ and longitudinal $L z$ lengths of the computational domain. Varying $L x, L y$, and $L z$ moves the radiation boundary closer to or farther away from the structure. It thus changes the size of the computational domain and has therefore a direct impact on the memory usage and simulation time. The normalized total emission rate and the computation time are plotted in Fig. 3(e) for an increasingly refined computational mesh, where the horizontal axis represents the number of consecutive mesh refinements (passes). Here, $L x=L y$ and $L z$ are constants with values given by the blue points in (f) and (g), respectively. For each mesh refinement, we compute the total electromagnetic energy stored in the system as well as the relative difference $\Delta$ Energy per pass. We define a relative energy difference convergence criterion of $\Delta$ Energy $<10^{-2}$, and convergence is obtained after seven consecutive mesh refinements (at the 8th pass) indicated by the blue circle in Fig. 3(e). We then present the influence of the lateral $(L x=L y)$ and longitudinal $(L z)$ computational domain length in Figs. 3(f,g), while keeping the other length constant. For each calculation point, the mesh is refined until the convergence criterion of $\Delta$ Energy $<10^{-2}$ is fulfilled, typically after at least 5-6 mesh refinements. While the time axis in (e) represents the calculation time for each mesh refinement, in (f,g), it represents the computational time for the final mesh refinement for which convergence is achieved.

When comparing the calculation time for the MM and FEM simulations in Fig. 3, we observe that the computation time for both methods is similar for the numerical parameters given by the blue circles. However, we first remark that the spontaneous emission rate computed using FEM in Figs. 3(f,g) displays subsequent variations of $\sim 20 \%$, where those for the MM are below $\sim 1 \%$. For the FEM simulations, a larger computation domain is thus required for convergence leading to significantly increased computation times as compared to the MM. Secondly, we remark that the calculation time of the full $\beta_{\mathrm{x}}$ map in Fig. 2 for the FEM is directly proportional to the number of grid points in the spatial map. However, for the MM, the eigenvalue problem yielding the eigenmodes only needs to be solved once, and subsequently $\beta_{\mathrm{x}}$ values for all $\mathbf{r}_{\perp}$ positions are computed without any notable computational effort from Eqs. $(27,28)$. We conclude that for the example in Fig. 2 of the calculation of the map of the spontaneous emission factor $\beta$, the MM both in terms of numerical accuracy and of computation time clearly outperforms the FEM.

\section{B. Benchmark II: Spontaneous Emission in a Rotationally Symmetric Nanowire}

Our new model is further compared and validated with modal method implementations in the literature that use expansions on individual coordinate variables [1526] that are directly compatible with Li's factorization rules. This is done by considering the case of spontaneous emission from a quantum emitter embedded in the infinite circular nanowire as depicted in the inset of Fig. 4. We calculate the coupling efficiency of the structure, $\beta=\Gamma_{1} / \Gamma_{\text {all }}=P_{1} / P_{\text {all }}$, where $\Gamma_{1}$ is the emission rate into the fundamental $\mathrm{HE}_{11}$ mode of the waveguide and $\Gamma_{\text {all }}$ is the total emission rate including both guided and radiation modes.

We consider a point dipole placed in the center of a GaAs $\left(n_{\mathrm{GaAs}}=3.45\right)$ nanowire surrounded by unbounded vacuum. We compute the spontaneous emission rate into specific guided modes (for which $\beta_{j}^{2}>n_{\text {clad }}^{2} k_{0}^{2}$ ) and into the radiation modes (defined by $\beta_{j}^{2}<n_{\text {clad }}^{2} k_{0}^{2}$ ) as a function of diameter. The spontaneous emission rate into the fundamental (second) guided mode $\Gamma_{1}\left(\Gamma_{2}\right)$ and into the radiation modes $\Gamma_{\mathrm{R}}$, normalized to the bulk emission rate, are presented in Fig. 4 together with the $\beta$ factor for unpolarized light. The calculations are performed using a truncation at $k_{\max }=200 k_{0}$. We used the nonuniform sampling scheme [25] with $N=600$ and $900 k$ space samples, corresponding to a total of 1200 and 1800 modes, respectively. The curves for different numbers of total modes are in a very good agreement indicating that 

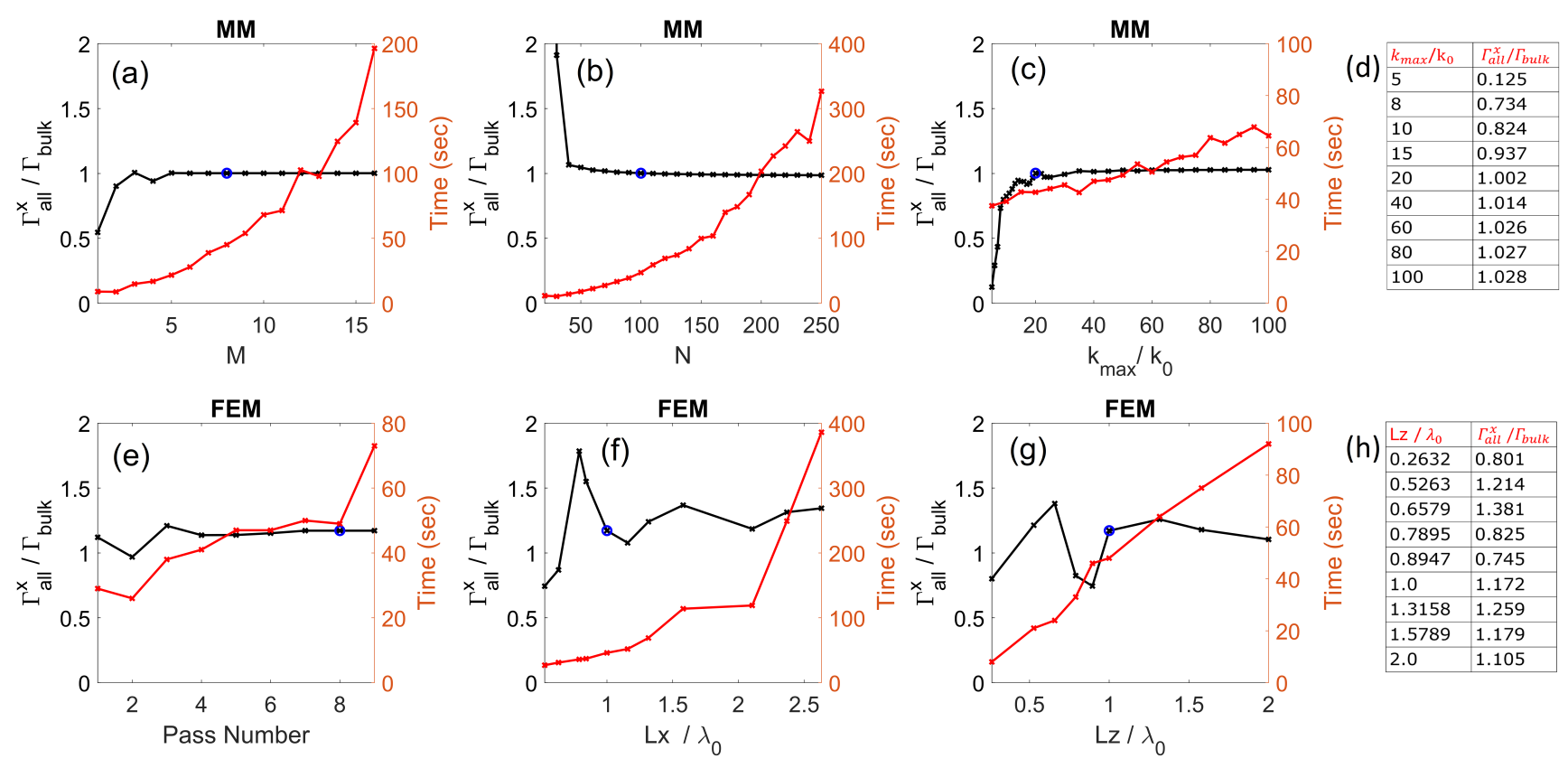

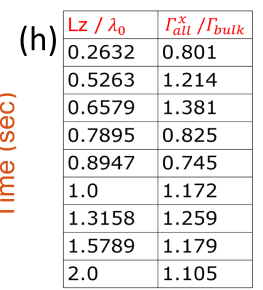

FIG. 3. Normalized total emission rate (black, left axis) and the computation time (red, right axis) with respect to the convergence parameters for $(a, b, c)$ MM and (e,f,g) FEM. For the MM, the parameters are (a) the total number of angular orders, (b) the number of $k$-space samples, (c) truncation $k_{\max }$ of the $k$ space; for FEM, (e) the number of consecutive mesh refinements applied (pass number) until the convergence criterion (see main text) is achieved, (f) the lateral, and (g) the longitudinal computational domain size. The blue circles correspond to the value used as a constant for the other sweeps. The data in $(\mathrm{c})$ and $(\mathrm{g})$ is presented in table formats in $(\mathrm{d})$ and $(\mathrm{h})$, respectively.

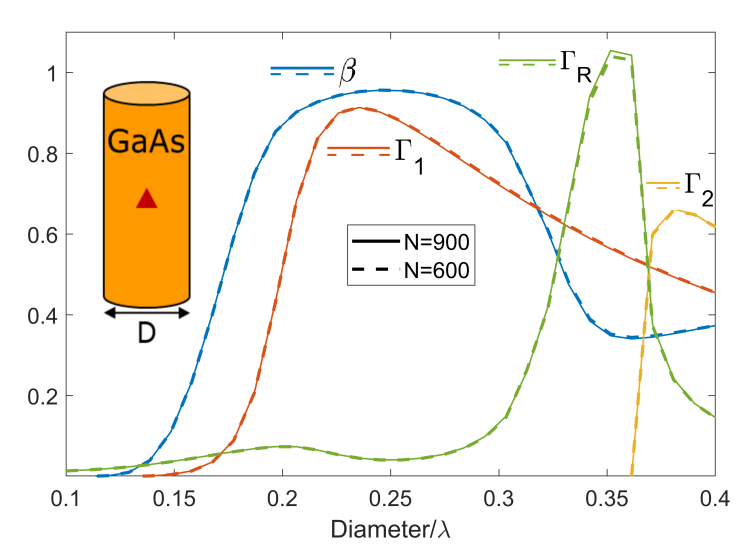

FIG. 4. Emission from an emitter in the center of an infinite vacuum-cladded nanowire as a function of diameter, normalized to the bulk emission rate $\Gamma_{\text {bulk. }}$. Spontaneous emission into the fundamental mode $\Gamma_{1}$ (red), the second guided mode $\Gamma_{2}$ (yellow), the radiation modes $\Gamma_{\mathrm{R}}$ (green), and the $\beta$ factor (blue) are shown. The rates are computed using $N=600$ (dashed) and $N=900$ (full) non-uniform $k$-space samples.

convergence has been reached, and we note that they also match with the results from the literature [5]. The calculation shows that for a diameter of $\sim 0.23 \lambda$, almost all the spontaneous emission is funnelled into the $\mathrm{HE}_{11}$ mode, which is one of the main assets of the photonic

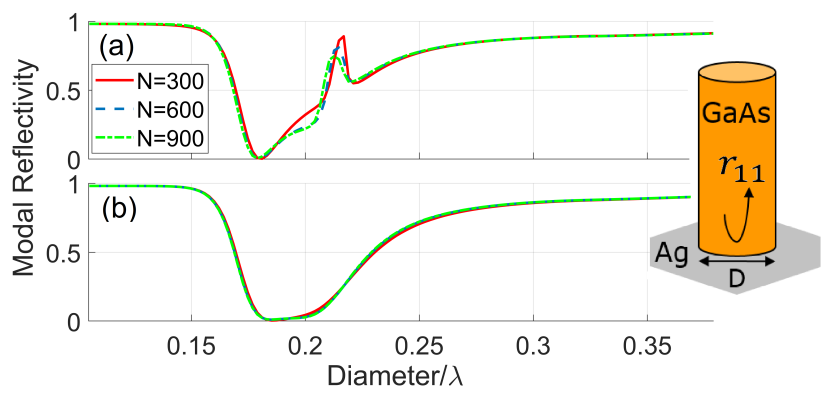

FIG. 5. Modal reflectivity $\left(\left|r_{11}\right|^{2}\right)$ of the fundamental mode of a GaAs nanowire - silver mirror interface as a function of nanowire diameter. Calculations are performed using (a) uniform sampling and (b) non-uniform sampling [25] of $k$ space for $N=300$ (red), $N=600$ (blue) and $N=900$ (green).

nanowire single-photon source $[6,7]$.

\section{Benchmark III: Reflection at a Nanowire-Metal Mirror Interface}

We next consider a GaAs nanowire $\left(n_{\mathrm{GaAs}}=3.5\right)$ attached to a silver mirror $[8,25]\left(n_{\mathrm{Ag}}=\sqrt{-41+2.5 i}\right)$ shown in the inset of Fig. 5, and we compute the modal reflectivity $\left|r_{11}\right|^{2}$ of the fundamental mode as a function of diameter. Figure 5(a) presents results for varying num- 
ber of modes obtained with a uniform sampling of the $k$ space, while the reflectivity in Fig. 5(b) is obtained with a non-uniform sampling scheme. The curves are obtained with different numbers of samples varying from 300 to 900 , as shown in the legend, and the $k$-space sampling is truncated at $k_{\max }=100 k_{0}$.

The reduction of the reflectivity at $D / \lambda \sim 0.18$ is caused by the excitation of surface plasmon polaritons [8], and here most of the energy is dissipated into radiation towards the sides. In this regime, simulations using uniform sampling converge slowly as the emission to the radiation modes, which occurs predominantly around $k_{\perp} \sim k_{0}$ is poorly resolved. In contrast, the non-uniform sampling scheme [25] provides a dense discretization of $k$-space around $k_{0}$ and as a result, the non-uniform sampling leads to significantly improved convergence as discussed further in Ref. [25]. Again, we note that very good agreement with Fig. 6 of Ref. [25] is obtained.

\section{DISCUSSION}

The direct access to the optical eigenmodes of a geometry with translational uniformity along a propagation axes enables highly efficient calculation of spontaneous emission $\beta$ factors and modal reflection coefficients, as required in numerous nanophotonic applications including single-photon source engineering. The sensitivity analysis of the emitter location in Fig. (2) requires a single computation of the eigenmodes, after which the spontaneous emission rate is computed for all positions $\mathbf{r}_{\perp}$ allowing the modal method simulations to be carried out within minutes. This ability represents a major asset of the modal method and makes it a preferred tool within single-photon source engineering [2-12]. On the other hand, other computational methods such as FEM and FDTD require new simulations for each position $\mathbf{r}_{\perp}$. They do not provide immediate access to the spontaneous emission rate into individual modes leading to added complexity $[13,14]$ in the evaluation of $\beta$ and corresponding simulation times from hours to days $[9,14]$.

The main novelty of this work is the introduction of a field expansion on TM and TE modes combined with an open geometry boundary condition leading to simple integrals for arbitrary orthogonal curvilinear coordinate systems, where the radial part can be computed efficiently by simply evaluating a basis function $\Psi\left(\mathbf{k}_{\perp}, \mathbf{r}_{\perp}\right)$ at discontinuity points. The efficient computation of the operator matrices relies on the low numerical cost of evaluating the basis functions. For the rotationally symmetric geometry, analytic solutions (Bessel functions) are available. For more general coordinate systems, analytic solutions may not be available and instead a numerical solution to Eq. (6) may be considered. In the case of the elliptical geometry, most mathematical programming languages, including MATLAB, do not include built-in support for Mathieu functions; nevertheless, they can be computed efficiently [32] using Bessel function expansions. In the case of the elliptical geometry, the presence of the coupling integral $\int_{0}^{2 \pi} V(v) V^{\prime}(v) d v$ in $(\mathrm{C} 7)$ remains, however it can be evaluated directly using the expansions (A19-A22) at no significant computational cost.

Whereas modal method implementations in the literature employing expansions on individual coordinate variables [15-26] are directly compatible with Li's factorization rules [19] for handling products of discontinuous functions, the implementation of the factorization rules in our formalism based on TE and TM basis modes is not straightforward. However, even without the use of Li's inverse factorization rule [19], we obtain equally good convergence as in simulations [25] implementing the inverse rule while avoiding the numerically costly matrix inversions of the inverse rule. A final asset of the formalism is the simplicity of its implementation. Indeed, a complete guide with all details of the derivations is included in the SI enabling graduate students the implementation of the presented method from scratch.

Finally, we note that while our new formalism allows for good convergence in the case of $\epsilon_{r}(u)$ profiles independent of $v$, where the operator matrix scales as $2 N M \times 2 N M$ and only a handful of angular orders $M$ are needed, the performance of the presented formalism extended to arbitrary $\epsilon_{r}\left(\mathbf{r}_{\perp}\right)$ profiles with a dense discretization of $k_{\perp}$ along two axes will suffer from the unfavorable $2 N^{2} \times 2 N^{2}$ scaling of the operator matrix. This poor performance of the modal method for such geometries is well-established [33] and represents a general challenge for all modal expansion methods.

The MM presented in this contribution is based on an expansion employing basis modes that are solutions to a geometry embedded in a homogeneous, isotropic, and unbounded background medium (e. g. vacuum). However, as a perspective for future work, expansions on more advanced basis modes can be envisioned. In the analysis of a ridge waveguide on top of a substrate [34, 35], convenient basis modes would be the solutions to the interface geometry consisting of only the substrate and air. Thus, only the ridge waveguide itself should be considered in the in-plane integrations, where $\Delta \eta_{r}=\Delta \epsilon_{r}=0$ in the substrate. While generalizations of the MM to both $u$ and $v$ dependent index profiles and to advanced basis modes are of great interest, they are beyond this article.

\section{CONCLUSION}

We have proposed a new modal method for open geometries introducing an expansion based on the TM and TE modes that are solutions to a geometry embedded in a homogeneous background medium, e. g. vacuum. The use of a TE and TM basis mode expansion leads to simple coupling integrals, which can be evaluated efficiently, leading to a rapid construction of the eigenvalue problem. We have benchmarked the method on highly challenging nanophotonic engineering problems, includ- 
ing calculations of spontaneous emission factors $\beta$ and of modal reflectivity coefficients, and we have shown that the computation time is significantly reduced compared to FEM simulations. While the method is particularly suitable for these advanced single-photon source engineering problems, it is sufficiently simple allowing for a straightforward and easy implementation.

\section{ACKNOWLEDGMENTS}

The authors acknowledge the support from Villum Fonden (VKR Center of Excellence NATEC-II, grant
$8692)$

\section{Appendix A: Basis Modes and Basis Functions}

\section{Orthogonality condition}

Using the bra-ket notation

$$
\langle\mathbf{a} \mid \mathbf{b}\rangle=\int\left[a_{u}(u, v) a_{v}(u, v)\right]\left[\begin{array}{l}
b_{u}(u, v) \\
b_{v}(u, v)
\end{array}\right] l_{u} l_{v} d u d v
$$

we obtain the following orthonormality relations for our basis modes

$$
\begin{aligned}
\left\langle\mathbf{f}_{\mathrm{TX}}\left(k_{\perp}^{\prime}\right) \mid \mathbf{f}_{\mathrm{TX}}\left(k_{\perp}\right)\right\rangle=\left\langle\mathbf{g}_{\mathrm{TX}}\left(k_{\perp}^{\prime}\right) \mid \mathbf{g}_{\mathrm{TX}}\left(k_{\perp}\right)\right\rangle= & \frac{1}{k_{\perp}^{\prime} k_{\perp}} \int\left[\left(\frac{\partial}{\partial u} \Psi_{\mathrm{TX}}\left(k_{\perp}^{\prime}\right)\right) \frac{l_{v}}{l_{u}}\left(\frac{\partial}{\partial u} \Psi_{\mathrm{TX}}\left(k_{\perp}\right)\right)\right. \\
& \left.+\left(\frac{\partial}{\partial v} \Psi_{\mathrm{TX}}\left(k_{\perp}^{\prime}\right)\right) \frac{l_{u}}{l_{v}}\left(\frac{\partial}{\partial v} \Psi_{\mathrm{TX}}\left(k_{\perp}\right)\right)\right] d u d v \\
= & -\frac{1}{k_{\perp}^{\prime} k_{\perp}} \int\left[\Psi_{\mathrm{TX}}\left(k_{\perp}^{\prime}\right)\left(\frac{\partial}{\partial u} \frac{l_{v}}{l_{u}} \frac{\partial}{\partial u} \Psi_{\mathrm{TX}}\left(k_{\perp}\right)\right)\right. \\
& \left.+\Psi_{\mathrm{TX}}\left(k_{\perp}^{\prime}\right)\left(\frac{\partial}{\partial v} \frac{l_{u}}{l_{v}} \frac{\partial}{\partial v} \Psi_{\mathrm{TX}}\left(k_{\perp}\right)\right)\right] d u d v \\
= & -\frac{1}{k_{\perp}^{\prime} k_{\perp}} \int l_{v} l_{u} \Psi_{\mathrm{TX}}\left(k_{\perp}^{\prime}\right) \nabla_{\perp}^{2} \Psi_{\mathrm{TX}}\left(k_{\perp}\right) d u d v \\
= & \frac{k_{\perp}}{k_{\perp}^{\prime}} \int l_{v} l_{u} \Psi_{\mathrm{TX}}\left(k_{\perp}^{\prime}\right) \Psi_{\mathrm{TX}}\left(k_{\perp}\right) d u d v=\delta\left(k_{\perp}^{\prime}-k_{\perp}\right),
\end{aligned}
$$

where we have used that our basis functions are normalized according to (7) in the last step. In the second step and in the following, we use integration by parts, where the boundary terms always vanish as discussed in SI Section 1.2 . Furthermore, we have

$$
\begin{aligned}
\left\langle\mathbf{f}_{\mathrm{TX}}\left(k_{\perp}^{\prime}\right) \mid \mathbf{g}_{\mathrm{TY}}\left(k_{\perp}\right)\right\rangle=\left\langle\mathbf{g}_{\mathrm{TX}}\left(k_{\perp}^{\prime}\right) \mid \mathbf{f}_{\mathrm{TY}}\left(k_{\perp}\right)\right\rangle= & \frac{1}{k_{\perp}^{\prime} k_{\perp}} \int\left[\left(\frac{\partial}{\partial u} \Psi_{\mathrm{TX}}\left(k_{\perp}^{\prime}\right)\right)\left(\frac{\partial}{\partial v} \Psi_{\mathrm{TY}}\left(k_{\perp}\right)\right)\right. \\
& \left.-\left(\frac{\partial}{\partial v} \Psi_{\mathrm{TX}}\left(k_{\perp}^{\prime}\right)\right)\left(\frac{\partial}{\partial u} \Psi_{\mathrm{TY}}\left(k_{\perp}\right)\right)\right] d u d v \\
= & -\frac{1}{k_{\perp}^{\prime} k_{\perp}} \int\left[\Psi_{\mathrm{TX}}\left(k_{\perp}^{\prime}\right)\left(\frac{\partial}{\partial u} \frac{\partial}{\partial v} \Psi_{\mathrm{TY}}\left(k_{\perp}\right)\right)\right. \\
& \left.-\Psi_{\mathrm{TX}}\left(k_{\perp}^{\prime}\right)\left(\frac{\partial}{\partial v} \frac{\partial}{\partial u} \Psi_{\mathrm{TY}}\left(k_{\perp}\right)\right)\right] d u d v=0 .
\end{aligned}
$$

\section{Circular cylindrical coordinates}

In the circular cylindrical coordinate system, the basis functions are separable, and we employ Bessel functions of the first kind and trigonometric functions, respectively, as radial and angular basis functions for circular cylindrical geometries, such that the TM basis functions are given by

$$
\begin{aligned}
& \Psi_{\mathrm{TM}, \mathrm{C} m}\left(k_{\perp}, m, r, \phi\right)=N\left(k_{\perp}\right)^{-1 / 2} J_{m}\left(k_{\perp} r\right) \cos (m \phi), \\
& \Psi_{\mathrm{TM}, \mathrm{S} m}\left(k_{\perp}, m, r, \phi\right)=N\left(k_{\perp}\right)^{-1 / 2} J_{m}\left(k_{\perp} r\right) \sin (m \phi),
\end{aligned}
$$

where $N\left(k_{\perp}\right)$ are normalization constants given below. While the basis functions Eqs. (A4,A5) for all $m$ represent a complete basis, the presence of the integral $\int_{0}^{2 \pi} V_{q}(v) V_{q^{\prime}}(v) d v$ in the operator matrix elements leads to a decoupling 
of $\Psi_{\mathrm{TM}, \mathrm{C} m}$ and $\Psi_{\mathrm{TM}, \mathrm{S} m}$ basis functions and of functions with different $m$. We may thus limit the calculation to the subset of basis functions consisting of a single angular order $m$ and a specific polarization $\Psi_{\mathrm{TM}, \mathrm{C} m}$ given by (A4). The associated TE basis function $\Psi_{\mathrm{TE}, \mathrm{S} m}$ is given by the RHS of (A5), where we in the following skip the Cm and $\mathrm{S} m$ subindices for simplicity. With the scale factors $l_{u}=1$ and $l_{v}=r$, the normalization factor $N_{m}\left(k_{\perp}\right)$ for $\mathrm{TM}$ polarization is obtained using

$$
\begin{aligned}
\int_{0}^{\infty} \int_{0}^{2 \pi} J_{m}\left(k_{\perp} r\right) \cos (m \phi) J_{m}\left(k_{\perp}^{\prime} r\right) \cos (m \phi) d \phi r d r & =\int_{0}^{2 \pi} \cos (m \phi) \cos (m \phi) d \phi \int_{0}^{\infty} J_{m}\left(k_{\perp} r\right) J_{m}\left(k_{\perp}^{\prime} r\right) r d r \\
& =N_{\phi, m} N_{r}\left(k_{\perp}\right) \delta\left(k_{\perp}-k_{\perp}^{\prime}\right) \equiv N_{m}\left(k_{\perp}\right) \delta\left(k_{\perp}-k_{\perp}^{\prime}\right)
\end{aligned}
$$

and similarly for TE polarization, such that

$$
\begin{aligned}
N_{\phi, m} & = \begin{cases}2 \pi & m=0 \\
\pi & m \neq 0\end{cases} \\
N_{r}\left(k_{\perp}\right) \delta\left(k_{\perp}-k_{\perp}^{\prime}\right) & =\int_{0}^{\infty} J_{m}\left(k_{\perp} r\right) J_{m}\left(k_{\perp}^{\prime} r\right) r d r=\frac{\delta\left(k_{\perp}-k_{\perp}^{\prime}\right)}{k_{\perp}} .
\end{aligned}
$$

The corresponding TM basis modes can be written as

$$
\begin{aligned}
f_{r, \mathrm{TM}}\left(k_{\perp}, m, r, \phi\right) & =\sqrt{\frac{1}{N_{m}\left(k_{\perp}\right)}} \frac{1}{k_{\perp}} \frac{\partial J_{m}\left(k_{\perp} r\right)}{\partial r} \cos (m \phi), \\
f_{\phi, \mathrm{TM}}\left(k_{\perp}, m, r, \phi\right) & =-\sqrt{\frac{1}{N_{m}\left(k_{\perp}\right)}} \frac{m}{k_{\perp} r} J_{m}\left(k_{\perp} r\right) \sin (m \phi), \\
g_{r, \mathrm{TM}}\left(k_{\perp}, m, r, \phi\right) & =-f_{\phi, \mathrm{TM}}\left(k_{\perp}, m, r, \phi\right), \\
g_{\phi, \mathrm{TM}}\left(k_{\perp}, m, r, \phi\right) & =f_{r, \mathrm{TM}}\left(k_{\perp}, m, r, \phi\right),
\end{aligned}
$$

and the TE basis modes are given by

$$
\begin{aligned}
f_{r, \mathrm{TE}}\left(k_{\perp}, m, r, \phi\right) & =\sqrt{\frac{1}{N_{m}\left(k_{\perp}\right)}} \frac{1}{k_{\perp}} \frac{\partial J_{m}\left(k_{\perp} r\right)}{\partial r} \sin (m \phi), \\
f_{\phi, \mathrm{TE}}\left(k_{\perp}, m, r, \phi\right) & =\sqrt{\frac{1}{N_{m}\left(k_{\perp}\right)}} \frac{m}{k_{\perp} r} J_{m}\left(k_{\perp} r\right) \cos (m \phi), \\
g_{r, \mathrm{TE}}\left(k_{\perp}, m, r, \phi\right) & =f_{\phi, \mathrm{TE}}\left(k_{\perp}, m, r, \phi\right), \\
g_{\phi, \mathrm{TE}}\left(k_{\perp}, m, r, \phi\right) & =-f_{r, \mathrm{TE}}\left(k_{\perp}, m, r, \phi\right) .
\end{aligned}
$$

\section{Elliptical cylindrical coordinates}

As in the case of circular cylindrical coordinates, the basis functions are also separable in the elliptical coordinate system, and the Helmholtz equation in an elliptical coordinate system with semi-focal distance $f$ reduces to [36]

$$
\begin{gathered}
\frac{\partial^{2} V(v)}{\partial v^{2}}+\left[a-\frac{k_{\perp}^{2} f^{2}}{2} \cos (2 v)\right] V(v)=0, \\
\frac{\partial^{2} U(u)}{\partial u^{2}}-\left[a-\frac{k_{\perp}^{2} f^{2}}{2} \cosh (2 u)\right] U(u)=0,
\end{gathered}
$$

where (A17) is the angular and (A18) is the radial (modified) Mathieu differential equation and $a$ is a separation constant. Several procedures [30, 32, 36-41] for solving the Mathieu differential equations have been suggested, and a useful MATLAB toolbox is also available [32]. We adopt the notation of Ref. [32] while modifying the normalization constants, with the help of the orthonormalization relation given in Ref. [42], to ensure that our normalization condition (7) is fulfilled. The solutions to Eqs. (A17,A18) can be classified in four symmetry groups, even-even, even-odd, odd-odd, odd-even, according to their parity along the $y$ and $x$ axis respectively. The angular Mathieu 
functions can then be written as expansions on the trigonometric functions as

$$
\begin{aligned}
& V_{\mathrm{ee}}\left(k_{\perp}, m, v\right)=\sum_{j=0}^{\infty} A_{\mathrm{ee}}^{(2 j)}\left(k_{\perp} f / 2, m\right) \cos (2 j v), \\
& V_{\mathrm{eo}}\left(k_{\perp}, m, v\right)=\sum_{j=0}^{\infty} A_{\mathrm{eo}}^{(2 j+1)}\left(k_{\perp} f / 2, m\right) \cos [(2 j+1) v], \\
& V_{\mathrm{oo}}\left(k_{\perp}, m, v\right)=\sum_{j=1}^{\infty} A_{\mathrm{oO}}^{(2 j)}\left(k_{\perp} f / 2, m\right) \sin (2 j v), \\
& V_{\mathrm{oe}}\left(k_{\perp}, m, v\right)=\sum_{j=0}^{\infty} A_{\mathrm{oe}}^{(2 j+1)}\left(k_{\perp} f / 2, m\right) \sin [(2 j+1) v],
\end{aligned}
$$

where $A_{\mathrm{xy}}^{(j)}$ represents expansion coefficients with the subindex e (o) denoting even (odd) parity and $m$ denotes the angular order.The $A$ coefficients are calculated by inserting the expansions into the differential equation and solving an eigenvalue problem $[32,36]$, and the expansion coefficients are normalized to ensure that

$$
\int_{0}^{2 \pi} V_{\mathrm{xy}}^{2}\left(k_{\perp}, m, v\right)=1 .
$$

The radial Mathieu function of the first kind can be written using Bessel functions as

$$
\begin{aligned}
& U_{\mathrm{ee}}\left(k_{\perp}, m, u\right)=\frac{\sqrt{k_{\perp}}}{A_{\mathrm{ee}}^{(0)}} \sum_{j=0}^{\infty}(-1)^{j} A_{\mathrm{ee}}^{(2 j)}\left(k_{\perp} f / 2, m\right) J_{j}\left(p_{1}\right) J_{j}\left(p_{2}\right), \\
& U_{\mathrm{eo}}\left(k_{\perp}, m, u\right)=\frac{\sqrt{k_{\perp}}}{A_{\mathrm{eO}}^{(1)}} \sum_{j=0}^{\infty}(-1)^{j} A_{\mathrm{eO}}^{(2 j+1)}\left(k_{\perp} f / 2, m\right)\left[J_{j}\left(p_{1}\right) J_{j+1}\left(p_{2}\right)+J_{j}\left(p_{2}\right) J_{j+1}\left(p_{1}\right)\right], \\
& U_{\mathrm{oo}}\left(k_{\perp}, m, u\right)=\frac{\sqrt{k_{\perp}}}{A_{\mathrm{oO}}^{(2)}} \sum_{j=1}^{\infty}(-1)^{j} A_{\mathrm{oO}}^{(2 j)}\left(k_{\perp} f / 2, m\right)\left[J_{j-1}\left(p_{1}\right) J_{j+1}\left(p_{2}\right)-J_{j-1}\left(p_{2}\right) J_{j+1}\left(p_{1}\right)\right], \\
& U_{\mathrm{oe}}\left(k_{\perp}, m, u\right)=\frac{\sqrt{k_{\perp}}}{A_{\mathrm{oe}}^{(1)}} \sum_{j=0}^{\infty}(-1)^{j} A_{\mathrm{oe}}^{(2 j+1)}\left(k_{\perp} f / 2, m\right)\left[J_{j}\left(p_{1}\right) J_{j+1}\left(p_{2}\right)-J_{j}\left(p_{2}\right) J_{j+1}\left(p_{1}\right)\right],
\end{aligned}
$$

where $p_{1}=\left(k_{\perp} f / 2\right) \exp (-u)$ and $p_{2}=\left(k_{\perp} f / 2\right) \exp (u)$. Note that the expansion coefficients are the same as for the angular and radial Mathieu functions. The integral $\int_{0}^{2 \pi} V_{\mathrm{xy}}(v) V_{\mathrm{x}^{\prime} \mathrm{y}^{\prime}}(v) d v$ leads to a decoupling of the four symmetry groups, and we may chose to limit the calculation to include only even-odd modes for the TM basis set $\Psi_{\mathrm{TM}, \mathrm{eo}}\left(k_{\perp}, m, u, v\right)=U_{\mathrm{eo}}\left(k_{\perp}, m, u\right) V_{\mathrm{eo}}\left(k_{\perp}, m, v\right)$ and odd-even modes $\Psi_{\mathrm{TE}, \mathrm{oe}}\left(k_{\perp}, m, u, v\right)=$ $U_{\mathrm{oe}}\left(k_{\perp}, m, u\right) V_{\mathrm{oe}}\left(k_{\perp}, m, v\right)$ for the TE basis. Consequently, the basis modes can be written as

$$
\begin{aligned}
f_{u, \mathrm{TM}}\left(k_{\perp}, m, u, v\right) & =\frac{1}{k_{\perp} l_{u}} \frac{\partial U_{\mathrm{eo}}\left(k_{\perp}, m, u\right)}{\partial u} V_{\mathrm{eo}}\left(k_{\perp}, m, v\right) \\
f_{v, \mathrm{TM}}\left(k_{\perp}, m, u, v\right) & =\frac{1}{k_{\perp} l_{v}} U_{\mathrm{eo}}\left(k_{\perp}, m, u\right) \frac{\partial V_{\mathrm{eo}}\left(k_{\perp}, m, v\right)}{\partial v}, \\
g_{u, \mathrm{TM}}\left(k_{\perp}, m, u, v\right) & =-f_{v, \mathrm{TM}}\left(k_{\perp}, m, u, v\right), \\
g_{v, \mathrm{TM}}\left(k_{\perp}, m, u, v\right) & =f_{u, \mathrm{TM}}\left(k_{\perp}, m, u, v\right), \\
f_{u, \mathrm{TE}}\left(k_{\perp}, m, u, v\right) & =\frac{1}{k_{\perp} l_{u}} \frac{\partial U_{\mathrm{oe}}\left(k_{\perp}, m, u\right)}{\partial u} V_{\mathrm{oe}}\left(k_{\perp}, m, v\right), \\
f_{v, \mathrm{TE}}\left(k_{\perp}, m, u, v\right) & =\frac{1}{k_{\perp} l_{v}} U_{\mathrm{oe}}\left(k_{\perp}, m, u\right) \frac{\partial V_{\mathrm{oe}}\left(k_{\perp}, m, v\right)}{\partial v} \\
g_{u, \mathrm{TE}}\left(k_{\perp}, m, u, v\right) & =f_{v, \mathrm{TE}}\left(k_{\perp}, m, u, v\right), \\
g_{v, \mathrm{TE}}\left(k_{\perp}, m, u, v\right) & =-f_{u, \mathrm{TE}}\left(k_{\perp}, m, u, v\right) .
\end{aligned}
$$

The representation Eqs. (A28-A35) represents the eo-oe class for TM and TE modes, respectively, which includes the fundamental $x$-polarized $\mathrm{HE}_{11}$ mode. To take all of the possible solutions into account, basis sets formed from the 
other three symmetry groups (oe-eo, ee-oo and oo-ee) should also be considered. Unlike the rotationally symmetric structure, the angular Mathieu functions with different angular order $m$ are not orthogonal, and in principle all angular orders $m$ should be included in a calculation. However, as discussed in Section III A, only a few orders are typically needed for convergence.

\section{Appendix B: Construction of Eigenvalue Problem}

The curl and Laplace operators in orthogonal curvilinear $(u, v, z)$ coordinates are given as

$$
\begin{aligned}
\nabla \times \mathbf{A}= & \left(\frac{1}{l_{v}} \frac{\partial A_{z}}{\partial v}-\frac{\partial A_{v}}{\partial z}\right) \hat{\mathbf{u}}+\left(\frac{\partial A_{u}}{\partial z}-\frac{1}{l_{u}} \frac{\partial A_{z}}{\partial u}\right) \hat{\mathbf{v}} \\
& +\frac{1}{l_{u} l_{v}}\left(\frac{\partial\left(l_{v} A_{v}\right)}{\partial u}-\frac{\partial\left(l_{u} A_{u}\right)}{\partial v}\right) \hat{\mathbf{z}} \\
\nabla_{\perp}^{2} f= & \frac{1}{l_{u} l_{v}}\left(\frac{\partial}{\partial u} \frac{l_{v}}{l_{u}} \frac{\partial}{\partial u}+\frac{\partial}{\partial v} \frac{l_{u}}{l_{v}} \frac{\partial}{\partial v}\right) f .
\end{aligned}
$$

Insertion of the curl operator Eq. (B1) into Maxwell's equations and elimination of the $e_{z}$ and $h_{z}$ components lead to the system of linear equations

$$
\begin{aligned}
& \hat{\mathbf{O}}_{\mathrm{eh}}\left[\begin{array}{l}
h_{u} \\
h_{v}
\end{array}\right]=\beta\left[\begin{array}{l}
e_{u} \\
e_{v}
\end{array}\right], \\
& \hat{\mathbf{O}}_{\text {he }}\left[\begin{array}{l}
e_{u} \\
e_{v}
\end{array}\right]=\beta\left[\begin{array}{l}
h_{u} \\
h_{v}
\end{array}\right],
\end{aligned}
$$

where the operators $\hat{\mathbf{O}}_{\mathrm{eh}}$ and $\hat{\mathbf{O}}_{\text {he }}$ using the scaled magnetic field discussed in Section II A and the relative impermittivity $\eta_{r}=1 / \epsilon_{r}$ are given by

$$
\begin{aligned}
& \hat{\mathbf{O}}_{\mathrm{eh}}=\left[\begin{array}{cc}
-\frac{1}{l_{u}} \frac{\partial}{\partial u} \frac{\eta_{r}}{k_{0}^{2}} \frac{1}{l_{u} l_{v}} \frac{\partial}{\partial v} l_{u} & 1+\frac{1}{l_{u}} \frac{\partial}{\partial u} \frac{\eta_{r}}{k_{0}^{2}} \frac{1}{l_{u} l_{v}} \frac{\partial}{\partial u} l_{v} \\
-1-\frac{1}{l_{v}} \frac{\partial}{\partial v} \frac{\eta_{r}}{k_{0}^{2}} \frac{1}{l_{u} l_{v}} \frac{\partial}{\partial v} l_{u} & \frac{1}{l_{v}} \frac{\partial}{\partial v} \frac{\eta_{r}}{k_{0}^{2}} \frac{1}{l_{u} l_{v}} \frac{\partial}{\partial u} l_{v}
\end{array}\right], \\
& \hat{\mathbf{O}}_{\mathrm{he}}=\left[\begin{array}{cc}
\frac{1}{l_{u}} \frac{\partial}{\partial u} \frac{1}{l_{u} l_{v}} \frac{\partial}{\partial v} l_{u} & -k_{0}^{2} \epsilon_{r}-\frac{1}{l_{u}} \frac{\partial}{\partial u} \frac{1}{l_{u} l_{v}} \frac{\partial}{\partial u} l_{v} \\
k_{0}^{2} \epsilon_{r}+\frac{1}{l_{v}} \frac{\partial}{\partial v} \frac{1}{l_{u} l_{v}} \frac{\partial}{\partial v} l_{u} & -\frac{1}{l_{v}} \frac{\partial}{\partial v} \frac{1}{l_{u} l_{v}} \frac{\partial}{\partial u} l_{v}
\end{array}\right] .
\end{aligned}
$$

We then write the transverse field expansions given by Eqs. $(12,13)$ as

$$
\begin{aligned}
& {\left[\begin{array}{l}
e_{u}(u, v) \\
e_{v}(u, v)
\end{array}\right]=\int\left[\begin{array}{ll}
f_{\mathrm{TM}, u}\left(k_{\perp}, u, v\right) & g_{\mathrm{TE}, u}\left(k_{\perp}, u, v\right) \\
f_{\mathrm{TM}, v}\left(k_{\perp}, u, v\right) & g_{\mathrm{TE}, v}\left(k_{\perp}, u, v\right)
\end{array}\right]\left[\begin{array}{l}
c_{\mathrm{TM}}^{E_{\perp}}\left(k_{\perp}\right) \\
c_{\mathrm{T}}^{E_{\perp}}\left(k_{\perp}\right)
\end{array}\right] d k_{\perp},} \\
& {\left[\begin{array}{l}
h_{u}(u, v) \\
h_{v}(u, v)
\end{array}\right]=\int\left[\begin{array}{ll}
g_{\mathrm{TM}, u}\left(k_{\perp}, u, v\right) & f_{\mathrm{TE}, u}\left(k_{\perp}, u, v\right) \\
g_{\mathrm{TM}, v}\left(k_{\perp}, u, v\right) & f_{\mathrm{TE}, v}\left(k_{\perp}, u, v\right)
\end{array}\right]\left[\begin{array}{l}
c_{\mathrm{TM}}^{H}\left(k_{\perp}\right) \\
c_{\mathrm{TE}}^{H}\left(k_{\perp}\right)
\end{array}\right] d k_{\perp} .}
\end{aligned}
$$

Insertion of Eqs. (B7,B8) into (B3) gives

$$
\int \hat{\mathbf{O}}_{\mathrm{eh}}\left[\begin{array}{ll}
g_{\mathrm{TM}, u}\left(k_{\perp}\right) & f_{\mathrm{TE}, u}\left(k_{\perp}\right) \\
g_{\mathrm{TM}, v}\left(k_{\perp}\right) & f_{\mathrm{TE}, v}\left(k_{\perp}\right)
\end{array}\right]\left[\begin{array}{c}
c_{\mathrm{TM}}^{H_{\perp}}\left(k_{\perp}\right) \\
c_{\mathrm{TE}}^{H \perp}\left(k_{\perp}\right)
\end{array}\right] d k_{\perp}=\beta \int\left[\begin{array}{ll}
f_{\mathrm{TM}, u}\left(k_{\perp}\right) & g_{\mathrm{TE}, u}\left(k_{\perp}\right) \\
f_{\mathrm{TM}, v}\left(k_{\perp}\right) & g_{\mathrm{TE}, v}\left(k_{\perp}\right)
\end{array}\right]\left[\begin{array}{c}
c_{\mathrm{TM}}^{E_{\perp}}\left(k_{\perp}\right) \\
c_{\perp}^{E_{\perp}}\left(k_{\perp}\right)
\end{array}\right] d k_{\perp}
$$

where the $u$ and $v$ dependencies have been skipped for simplicity. Multiplying (B9) with $\int\left[\begin{array}{cc}f_{\mathrm{TM}, u}\left(k_{\perp}^{\prime}\right) & f_{\mathrm{TM}, v}\left(k_{\perp}^{\prime}\right) \\ g_{\mathrm{TE}, u}\left(k_{\perp}^{\prime}\right) & g_{\mathrm{TE}, v}\left(k_{\perp}^{\prime}\right)\end{array}\right] d \mathbf{r}_{\perp}$ and carrying out the RHS $k_{\perp}$ integration using the orthonormalization conditions Eqs. (A2,A3) leads to

$$
\int\left[\begin{array}{ll}
f_{\mathrm{TM}, u}\left(k_{\perp}^{\prime}\right) & f_{\mathrm{TM}, v}\left(k_{\perp}^{\prime}\right) \\
g_{\mathrm{TE}, u}\left(k_{\perp}^{\prime}\right) & g_{\mathrm{TE}, v}\left(k_{\perp}^{\prime}\right)
\end{array}\right] \hat{\mathbf{O}}_{\mathrm{eh}}\left[\begin{array}{ll}
g_{\mathrm{TM}, u}\left(k_{\perp}\right) & f_{\mathrm{TE}, u}\left(k_{\perp}\right) \\
g_{\mathrm{TM}, v}\left(k_{\perp}\right) & f_{\mathrm{TE}, v}\left(k_{\perp}\right)
\end{array}\right] \times\left[\begin{array}{c}
c_{\mathrm{TM}}^{H_{\perp}}\left(k_{\perp}\right) \\
c_{\mathrm{TE}}^{H}\left(k_{\perp}\right)
\end{array}\right] d \mathbf{r}_{\perp} d k_{\perp}=\beta\left[\begin{array}{c}
c_{\mathrm{TM}}^{E_{\perp}}\left(k_{\perp}^{\prime}\right) \\
c_{\mathrm{TE}}^{E_{\perp}}\left(k_{\perp}^{\prime}\right)
\end{array}\right] .
$$

This can be written as

$$
\int \overline{\bar{O}}_{\mathrm{eh}}\left(k_{\perp}^{\prime}, k_{\perp}\right)\left[\begin{array}{c}
c_{\mathrm{TM}}^{H_{\perp}}\left(k_{\perp}\right) \\
c_{\mathrm{TE}}^{H_{\perp}}\left(k_{\perp}\right)
\end{array}\right] d k_{\perp}=\beta\left[\begin{array}{c}
c_{\mathrm{TM}}^{E_{\perp}}\left(k_{\perp}^{\prime}\right) \\
c_{\mathrm{TE}}^{E_{\perp}}\left(k_{\perp}^{\prime}\right)
\end{array}\right]
$$


where we have defined the operator matrix $\overline{\bar{O}}_{\text {eh }}$ representing the $\hat{\mathbf{O}}_{\text {eh }}$ operator consisting of the four TX-TX coupling blocks as

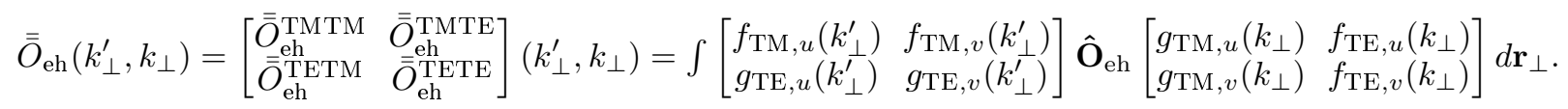

The TM-TM block is derived as follows:

$$
\begin{aligned}
& \overline{\bar{O}}_{\mathrm{eh}}^{\mathrm{TMTM}}\left(k_{\perp}^{\prime}, k_{\perp}\right)=\int\left[f_{\mathrm{TM}, u}\left(k_{\perp}^{\prime}\right) f_{\mathrm{TM}, v}\left(k_{\perp}^{\prime}\right)\right] \hat{\mathbf{O}}_{\mathrm{eh}}\left[\begin{array}{l}
g_{\mathrm{TM}, u}\left(k_{\perp}\right) \\
g_{\mathrm{TM}, v}\left(k_{\perp}\right)
\end{array}\right] d \mathbf{r}_{\perp} \\
& =\frac{1}{k_{\perp}^{\prime} k_{\perp}} \int l_{u} l_{v}\left(\left[\begin{array}{ll}
\frac{1}{l_{u}} \frac{\partial}{\partial u} & \frac{1}{l_{v}} \frac{\partial}{\partial v}
\end{array}\right] \Psi_{\mathrm{TM}}\left(k_{\perp}^{\prime}\right)\right)\left[\begin{array}{cc}
-\frac{1}{l_{u}} \frac{\partial}{\partial u} \frac{\eta_{r}}{k_{0}^{2}} \frac{1}{l_{u} l_{v}} \frac{\partial}{\partial v} l_{u} & 1+\frac{1}{l_{u}} \frac{\partial}{\partial u} \frac{\eta_{r}}{k_{0}^{2}} \frac{1}{l_{u} l_{v}} \frac{\partial}{\partial u} l_{v} \\
-1-\frac{1}{l_{v}} \frac{\partial}{\partial v} \frac{\eta_{r}}{k_{0}^{2}} \frac{1}{l_{u} l_{v}} \frac{\partial}{\partial v} l_{u} & \frac{1}{l_{v}} \frac{\partial}{\partial v} \frac{\eta_{r}}{k_{0}^{2}} \frac{1}{l_{u} l_{v}} \frac{\partial}{\partial u} l_{v}
\end{array}\right] \\
& \times\left[\begin{array}{c}
-\frac{1}{l_{v}} \frac{\partial}{\partial v} \\
\frac{1}{l_{u}} \frac{\partial}{\partial u}
\end{array}\right] \Psi_{\mathrm{TM}}\left(k_{\perp}\right) d u d v \\
& =\frac{1}{k_{\perp}^{\prime} k_{\perp}} \int l_{u} l_{v}\left(\left[\begin{array}{ll}
\frac{1}{l_{u}} \frac{\partial}{\partial u} & \frac{1}{l_{v}} \frac{\partial}{\partial v}
\end{array}\right] \Psi_{\mathrm{TM}}\left(k_{\perp}^{\prime}\right)\right)\left[\begin{array}{l}
\frac{1}{l_{u}} \frac{\partial}{\partial u} \frac{\eta_{r}}{k_{0}^{2}} \nabla_{\perp}^{2}+\frac{1}{l_{u}} \frac{\partial}{\partial u} \\
\frac{1}{l_{v}} \frac{\partial}{\partial v}+\frac{1}{l_{v}} \frac{\partial}{\partial v} \frac{\eta_{r}}{k_{0}^{2}} \nabla_{\perp}^{2}
\end{array}\right] \Psi_{\mathrm{TM}}\left(k_{\perp}\right) d u d v \\
& =-\frac{1}{k_{\perp}^{\prime} k_{\perp}} \int\left[\left(\frac{\partial}{\partial u} \frac{l_{v}}{l_{u}} \frac{\partial}{\partial u}+\frac{\partial}{\partial v} \frac{l_{u}}{l_{v}} \frac{\partial}{\partial v}\right) \Psi_{\mathrm{TM}}\left(k_{\perp}^{\prime}\right)\right]\left[1+\frac{\eta_{r}}{k_{0}^{2}} \nabla_{\perp}^{2}\right] \Psi_{\mathrm{TM}}\left(k_{\perp}\right) d u d v \\
& =-k_{\perp}^{\prime} k_{\perp} \int l_{u} l_{v} \Psi_{\mathrm{TM}}\left(k_{\perp}^{\prime}\right) \frac{\eta_{r}}{k_{0}^{2}} \Psi_{\mathrm{TM}}\left(k_{\perp}\right) d u d v+\delta\left(k_{\perp}^{\prime}-k_{\perp}\right) \\
& =-k_{\perp}^{\prime} k_{\perp} \int_{0}^{u_{b}} \int_{0}^{2 \pi} l_{u} l_{v} \Psi_{\mathrm{TM}}\left(k_{\perp}^{\prime}\right) \frac{\Delta \eta_{r}}{k_{0}^{2}} \Psi_{\mathrm{TM}}\left(k_{\perp}\right) d u d v+\left(1-\frac{\eta_{r, b} k_{\perp}^{2}}{k_{0}^{2}}\right) \delta\left(k_{\perp}^{\prime}-k_{\perp}\right) .
\end{aligned}
$$

The TM-TM contribution to the LHS of (B11) then becomes

$$
\begin{aligned}
\int \overline{\bar{O}}_{\mathrm{eh}}^{\mathrm{TMTM}}\left(k_{\perp}^{\prime}, k_{\perp}\right) c_{\mathrm{TM}}^{H_{\perp}}\left(k_{\perp}\right) d k_{\perp}= & -k_{\perp}^{\prime} k_{\perp} \int_{0}^{u_{b}} \int_{0}^{2 \pi}\left[l_{u} l_{v} \Psi_{\mathrm{TM}}\left(k_{\perp}^{\prime}\right) \frac{\Delta \eta_{r}}{k_{0}^{2}} \Psi_{\mathrm{TM}}\left(k_{\perp}\right) d u d v\right. \\
& \left.+\left(\frac{\eta_{r, b} k_{\perp}^{2}}{k_{0}^{2}}-1\right) \delta\left(k_{\perp}^{\prime}-k_{\perp}\right)\right] c_{\mathrm{TM}}^{H_{\perp}}\left(k_{\perp}\right) d k_{\perp} .
\end{aligned}
$$

Finally, by introducing the discretization (20) as

$$
\int \overline{\bar{O}}_{\mathrm{eh}}^{\mathrm{TMTM}}\left(k_{\perp}^{\prime}, k_{\perp}\right) c_{\mathrm{TM}}^{H_{\perp}}\left(k_{\perp}\right) d k_{\perp} \rightarrow \sum_{q=1}^{q=N} \overline{\bar{O}}_{\mathrm{eh}, \mathrm{q}^{\prime} \mathrm{q}}^{\mathrm{TMTM}} c_{\mathrm{TM}}^{H_{\perp}}\left(k_{\perp, q}\right)
$$

the operator matrix element (24) is obtained.

The detailed derivations of the remaining matrix elements are found in SI Section 3.1. The resulting matrix elements are

$$
\begin{aligned}
\overline{\bar{O}}_{\mathrm{eh}, q^{\prime} q}^{\mathrm{TETE}}= & \delta_{q^{\prime} q}, \\
\overline{\bar{O}}_{\mathrm{eh}, q^{\prime} q}^{\mathrm{TETM}}= & \overline{\bar{O}}_{\mathrm{eh}, q^{\prime} q}^{\mathrm{TMTE}}=0 \\
\overline{\bar{O}}_{\mathrm{he}, q^{\prime} q}^{\mathrm{TMTM}}= & \frac{k_{\perp, q}}{k_{\perp, q^{\prime}}} \int_{0}^{u_{b}} \int_{0}^{2 \pi} l_{u} l_{v} \Psi_{\mathrm{TM}, q^{\prime}}(u, v) \Delta \epsilon_{r}(u) k_{0}^{2} \Psi_{\mathrm{TM}, q}(u, v) d v d u \Delta k_{\perp, q}+\delta_{q^{\prime} q} k_{0}^{2} \epsilon_{r, b} \\
& -\frac{1}{k_{\perp, q^{\prime}} k_{\perp, q}} \int_{0}^{u_{b}} \int_{0}^{2 \pi} \Psi_{\mathrm{TM}, q^{\prime}}(u, v) k_{0}^{2}\left(\frac{\partial \epsilon_{r}(u)}{\partial u}\right) \frac{l_{v}}{l_{u}} \frac{\partial}{\partial u} \Psi_{\mathrm{TM}, q}(u, v) d v d u \Delta k_{\perp, q}, \\
\overline{\bar{O}}_{\mathrm{he}, q^{\prime} q}^{\mathrm{TETE}}= & \frac{k_{\perp, q}}{k_{\perp, q^{\prime}}} \int_{0}^{u_{b}} \int_{0}^{2 \pi} l_{u} l_{v} \Psi_{\mathrm{TE}, q^{\prime}}(u, v) \Delta \epsilon_{r}(u) k_{0}^{2} \Psi_{\mathrm{TE}, q}(u, v) d v d u \Delta k_{\perp, q}+\delta_{q^{\prime} q}\left(k_{0}^{2} \epsilon_{r, b}-k_{\perp, q}^{2}\right) \\
& -\frac{1}{k_{\perp, q^{\prime}} k_{\perp, q}} \int_{0}^{u_{b}} \int_{0}^{2 \pi} \Psi_{\mathrm{TE}, q^{\prime}}(u, v) k_{0}^{2}\left(\frac{\partial \epsilon_{r}(u)}{\partial u}\right) \frac{l_{v}}{l_{u}} \frac{\partial}{\partial u} \Psi_{\mathrm{TE}, q}(u, v) d v d u \Delta k_{\perp, q}, \\
\overline{\bar{O}}_{\mathrm{he}, q^{\prime} q}^{\mathrm{TMTE}=} & -\frac{1}{k_{\perp, q^{\prime}} k_{\perp, q}} \int_{0}^{u_{b}} \int_{0}^{2 \pi} \Psi_{\mathrm{TM}, q^{\prime}}(u, v) k_{0}^{2}\left(\frac{\partial \epsilon_{r}(u)}{\partial u}\right) \frac{\partial}{\partial v} \Psi_{\mathrm{TE}, q}(u, v) d v d u \Delta k_{\perp, q}, \\
\overline{\bar{O}}_{\mathrm{he}, q^{\prime} q}^{\mathrm{TETM}}= & \frac{1}{k_{\perp, q^{\prime}} k_{\perp, q}} \int_{0}^{u_{b}} \int_{0}^{2 \pi} \Psi_{\mathrm{TE}, q^{\prime}}(u, v) k_{0}^{2}\left(\frac{\partial \epsilon_{r}(u)}{\partial u}\right) \frac{\partial}{\partial v} \Psi_{\mathrm{TM}, q}(u, v) d v d u \Delta k_{\perp, q},
\end{aligned}
$$


where $\epsilon_{r, b}$ is the cladding permittivity and $\Delta \epsilon_{r}(u)=\epsilon_{r}(u)-\epsilon_{r, b}$. All the matrix elements above only involve integrals of the form

$$
\begin{aligned}
& \int_{u_{a}}^{u_{b}} \int_{0}^{2 \pi} l_{u} l_{v} \Psi_{q^{\prime}}(u, v) \Psi_{q}(u, v) d u d v, \\
& \int_{u_{a}}^{u_{b}} \int_{0}^{2 \pi} \frac{l_{v}}{l_{u}} \Psi_{q^{\prime}}(u, v)\left(\frac{\partial \epsilon_{r}(u)}{\partial u}\right) \frac{\partial}{\partial u} \Psi_{q}(u, v) d u d v, \\
& \int_{u_{a}}^{u_{b}} \int_{0}^{2 \pi} \frac{l_{v}}{l_{u}} \Psi_{q^{\prime}}(u, v)\left(\frac{\partial \epsilon_{r}(u)}{\partial u}\right) \frac{\partial}{\partial v} \Psi_{q}(u, v) d u d v .
\end{aligned}
$$

We note that the radial integration in Eqs. (B23,B24) reduces to direct evaluation of the basis functions and their derivatives at the $\epsilon_{r}$ discontinuity points due to $\partial \epsilon_{r} / \partial u$ term in the case of a piece-wise constant permittivity profile. An analytical expression for the integration in Eq. (B22) is derived in the next section.

\section{Appendix C: Evaluation of Coupling Integrals}

We assume two different solutions $\Psi$ and $\Psi^{\prime}$ to the in-plane Helmholtz equation (6) fulfilling

$$
\begin{aligned}
\nabla^{2} \Psi(u, v)+k_{\perp}^{2} \Psi(u, v) & =0, \\
\nabla^{2} \Psi^{\prime}(u, v)+k_{\perp}^{\prime 2} \Psi^{\prime}(u, v) & =0 .
\end{aligned}
$$

Skipping the coordinate variables for simplicity, the operation $\Psi^{\prime} \cdot(C 1)-\Psi \cdot(C 2)$ leads to

$$
\Psi^{\prime} \nabla^{2} \Psi-\Psi \nabla^{2} \Psi^{\prime}+\left(k_{\perp}^{2}-k_{\perp}^{2}\right) \Psi \Psi^{\prime}=0
$$

which can be rewritten as

$$
\frac{\partial}{\partial u}\left(\Psi^{\prime} \frac{l_{v}}{l_{u}} \frac{\partial}{\partial u} \Psi-\Psi \frac{l_{v}}{l_{u}} \frac{\partial}{\partial u} \Psi^{\prime}\right)+\frac{\partial}{\partial v}\left(\Psi^{\prime} \frac{l_{u}}{l_{v}} \frac{\partial}{\partial v} \Psi-\Psi \frac{l_{u}}{l_{v}} \frac{\partial}{\partial v} \Psi^{\prime}\right)+l_{u} l_{v}\left(k_{\perp}^{2}-k_{\perp}^{\prime 2}\right) \Psi \Psi^{\prime}=0 .
$$

We integrate both sides along $u$ from $u_{a}$ to $u_{b}$ and along $v$ from 0 to $2 \pi$ and obtain

$$
\begin{aligned}
\left(k_{\perp}^{2}-k_{\perp}^{2}\right) \int_{u_{a}}^{u_{b}} \int_{0}^{2 \pi} l_{u} l_{v} \Psi \Psi^{\prime} d u d v & =\int_{0}^{2 \pi}\left[\Psi \frac{l_{v}}{l_{u}} \frac{\partial}{\partial u} \Psi^{\prime}-\Psi^{\prime} \frac{l_{v}}{l_{u}} \frac{\partial}{\partial u} \Psi\right]_{u=u_{a}}^{u=u_{b}} d v+\int_{u_{a}}^{u_{b}}\left[\Psi \frac{l_{u}}{l_{v}} \frac{\partial}{\partial v} \Psi^{\prime}-\Psi^{\prime} \frac{l_{u}}{l_{v}} \frac{\partial}{\partial v} \Psi\right]_{v=0}^{v=2 \pi} d u \\
& =\int_{0}^{2 \pi}\left[\Psi \frac{l_{v}}{l_{u}} \frac{\partial}{\partial u} \Psi^{\prime}-\Psi^{\prime} \frac{l_{v}}{l_{u}} \frac{\partial}{\partial u} \Psi\right]_{u=u_{a}}^{u=u_{b}} d v,
\end{aligned}
$$

where we have exploited the angular periodicity of the basis function, $\Psi(u, v+2 \pi)=\Psi(u, v)$, in the last step. If separable basis functions are available, we can then expand our solutions as $\Psi(u, v)=U(u) V(v)$ and $\Psi^{\prime}(u, v)=$ $U^{\prime}(u) V^{\prime}(v)$. We then obtain

$$
\begin{aligned}
\left(k_{\perp}^{2}-\right. & \left.k_{\perp}^{\prime 2}\right) \int_{u_{a}}^{u_{b}} \int_{0}^{2 \pi} l_{u} l_{v} U(u) V(v) U^{\prime}(u) V^{\prime}(v) d v d u \\
= & {\left[U\left(u_{b}\right) \frac{\partial U^{\prime}\left(u_{b}\right)}{\partial u}\right] \int_{0}^{2 \pi} \frac{l_{v}\left(u_{b}, v\right)}{l_{u}\left(u_{b}, v\right)} V(v) V^{\prime}(v) d v-\left[U^{\prime}\left(u_{b}\right) \frac{\partial U\left(u_{b}\right)}{\partial u}\right] \int_{0}^{2 \pi} \frac{l_{v}\left(u_{b}, v\right)}{l_{u}\left(u_{b}, v\right)} V(v) V^{\prime}(v) d v } \\
& -\left[U\left(u_{a}\right) \frac{\partial U^{\prime}\left(u_{a}\right)}{\partial u}\right] \int_{0}^{2 \pi} \frac{l_{v}\left(u_{a}, v\right)}{l_{u}\left(u_{a}, v\right)} V(v) V^{\prime}(v) d v+\left[U^{\prime}\left(u_{a}\right) \frac{\partial U\left(u_{a}\right)}{\partial u}\right] \int_{0}^{2 \pi} \frac{l_{v}\left(u_{a}, v\right)}{l_{u}\left(u_{a}, v\right)} V(v) V^{\prime}(v) d v .
\end{aligned}
$$

Note that in elliptical coordinates, we have $l_{u}=l_{v}$, which leads to

$$
\left(k_{\perp}^{2}-k_{\perp}^{\prime 2}\right) \int_{u_{a}}^{u_{b}} \int_{0}^{2 \pi} l_{u} l_{v} U(u) V(v) U^{\prime}(u) V^{\prime}(v) d v d u=\left[U(u) \frac{\partial U^{\prime}(u)}{\partial u}-U^{\prime}(u) \frac{\partial U(u)}{\partial u}\right]_{u=u_{a}}^{u=u_{b}} \int_{0}^{2 \pi} V(v) V^{\prime}(v) d v
$$

[1] Y. Yu, W. Xue, E. Semenova, K. Yvind, and J. Mork, Demonstration of a self-pulsing photonic crystal Fano laser, Nat. Photonics 11, 81 (2017). 
[2] I. Aharonovich, D. Englund, and M. Toth, Solid-state single-photon emitters, Nat. Photonics 10, 631 (2016).

[3] N. Gregersen, P. Kaer, and J. Mørk, Modeling and Design of High-Efficiency Single-Photon Sources, IEEE J. Sel. Top. Quantum Electron. 19, 9000516 (2013).

[4] A. D. Osterkryger, J.-M. Gérard, J. Claudon, and N. Gregersen, Photonic "hourglass" design for efficient quantum light emission, Opt. Lett. 44, 2617 (2019).

[5] Y.-R. Nowicki-Bringuier, R. Hahner, J. Claudon, G. Lecamp, P. Lalanne, and J. Gérard, A novel highefficiency single-mode single photon source, in Annales de Physique, Vol. 32 (EDP Sciences, 2007) pp. 151-154.

[6] I. Friedler, C. Sauvan, J.-P. Hugonin, P. Lalanne, J. Claudon, and J.-M. Gérard, Solid-state single photon sources: the nanowire antenna, Optics express 17, 2095 (2009).

[7] J. Claudon, J. Bleuse, N. S. Malik, M. Bazin, P. Jaffrennou, N. Gregersen, C. Sauvan, P. Lalanne, and J.-M. Gérard, A highly efficient single-photon source based on a quantum dot in a photonic nanowire, Nature Photonics 4, 174 (2010).

[8] I. Friedler, P. Lalanne, J. P. Hugonin, J. Claudon, J. M. Gérard, A. Beveratos, and I. Robert-Philip, Efficient photonic mirrors for semiconductor nanowires, Opt. Lett. 33, 2635 (2008).

[9] M. Munsch, J. Claudon, J. Bleuse, N. S. Malik, E. Dupuy, J.-M. Gérard, Y. Chen, N. Gregersen, and J. Mørk, Linearly polarized, single-mode spontaneous emission in a photonic nanowire, Phys. Rev. Lett. 108, 077405 (2012).

[10] S. Reitzenstein and A. Forchel, Quantum dot micropillars, J. Phys. D. Appl. Phys. 43, 033001 (2010).

[11] X. Ding, Y. He, Z.-C. Duan, N. Gregersen, M.-C. Chen, S. Unsleber, S. Maier, C. Schneider, M. Kamp, S. Höfling, C.-Y. Lu, and J.-W. Pan, On-Demand Single Photons with High Extraction Efficiency and Near-Unity Indistinguishability from a Resonantly Driven Quantum Dot in a Micropillar, Phys. Rev. Lett. 116, 020401 (2016).

[12] H. Wang, Y.-M. He, T.-H. Chung, H. Hu, Y. Yu, S. Chen, X. Ding, M.-C. Chen, J. Qin, X. Yang, R.-Z. Liu, Z.C. Duan, J.-P. Li, S. Gerhardt, K. Winkler, J. Jurkat, L.-J. Wang, N. Gregersen, Y.-H. Huo, Q. Dai, S. Yu, S. Höfling, C.-Y. Lu, and J.-W. Pan, Towards optimal single-photon sources from polarized microcavities, Nat. Photonics 13, 770 (2019).

[13] Y. Chen, T. R. Nielsen, N. Gregersen, P. Lodahl, and J. Mørk, Finite-element modeling of spontaneous emission of a quantum emitter at nanoscale proximity to plasmonic waveguides, Phys. Rev. B 81, 125431 (2010), arXiv:0909.3233.

[14] A. Javadi, S. Mahmoodian, I. Söllner, and P. Lodahl, Numerical modeling of the coupling efficiency of single quantum emitters in photonic-crystal waveguides, J. Opt. Soc. Am. B 35, 514 (2018).

[15] M. G. Moharam, E. B. Grann, D. A. Pommet, and T. K. Gaylord, Formulation for stable and efficient implementation of the rigorous coupled-wave analysis of binary gratings, J. Opt. Soc. Am. A 12, 1068 (1995).

[16] E. Silberstein, P. Lalanne, J.-P. Hugonin, and Q. Cao, Use of grating theories in integrated optics., J. Opt. Soc. Am. A 18, 2865 (2001).

[17] P. Lalanne and G. M. Morris, Highly improved convergence of the coupled-wave method for TM polarization, J. Opt. Soc. Am. A 13, 779 (1996).

[18] G. Granet and B. Guizal, Efficient implementation of the coupled-wave method for metallic lamellar gratings in TM polarization, J. Opt. Soc. Am. A 13, 1019 (1996).

[19] L. Li, Use of Fourier series in the analysis of discontinuous periodic structures, J. Opt. Soc. Am. A 13, 1870 (1996).

[20] L. Li, New formulation of the Fourier modal method for crossed surface-relief gratings, J. Opt. Soc. Am. A 14, 2758 (1997).

[21] M. Neviere and E. Popov, Light Propagation in Periodic Media: Differential Theory and Design (Marcel Dekker, New York, 2003).

[22] T. Schuster, J. Ruoff, N. Kerwien, S. Rafler, and W. Osten, Normal vector method for convergence improvement using the RCWA for crossed gratings, Journal of the Optical Society of America A 24, 2880 (2007).

[23] J. P. Hugonin and P. Lalanne, Perfectly matched layers as nonlinear coordinate transforms: a generalized formalization, J. Opt. Soc. Am. A 22, 1844 (2005).

[24] N. Bonod, E. Popov, and M. Nevière, Differential theory of diffraction by finite cylindrical objects, Journal of the Optical Society of America A 22, 481 (2005).

[25] T. Häyrynen, J. R. de Lasson, and N. Gregersen, Opengeometry Fourier modal method: Modeling nanophotonic structures in infinite domains, J. Opt. Soc. Am. A 33, 1298 (2016), arXiv:1603.05386.

[26] T. Häyrynen, A. D. Osterkryger, J. R. de Lasson, and N. Gregersen, Modeling open nanophotonic systems using the Fourier modal method: generalization to $3 \mathrm{D}$ Cartesian coordinates, J. Opt. Soc. Am. A 34, 1632 (2017).

[27] L. Li, Formulation and comparison of two recursive matrix algorithms for modeling layered diffraction gratings, J. Opt. Soc. Am. A 13, 1024 (1996).

[28] A. V. Lavrinenko, J. Lægsgaard, N. Gregersen, F. Schmidt, and T. Søndergaard, Numerical methods in photonics (CRC Press, 2014).

[29] A. Yariv, Optical Electronics in Modern Communications, 5th ed. (Oxford University Press, 1997).

[30] P. M. Morse and H. Feshbach, Methods of Theoretical Physics. Vol. 1-2 (McGraw-Hill, 1953).

[31] L. Novotny and B. Hecht, Principles of nano-optics (Cambridge university press, 2012).

[32] E. Cojocaru, Mathieu functions computational toolbox implemented in Matlab, arXiv 0811.1970 (2008), arXiv:0811.1970.

[33] J. R. de Lasson, L. H. Frandsen, P. Gutsche, S. Burger, O. S. Kim, O. Breinbjerg, A. Ivinskaya, F. Wang, O. Sigmund, T. Häyrynen, A. V. Lavrinenko, J. Mørk, and $\mathrm{N}$. Gregersen, Benchmarking five numerical simulation techniques for computing resonance wavelengths and quality factors in photonic crystal membrane line defect cavities, Opt. Express 26, 11366 (2018).

[34] P. Stepanov, A. Delga, X. Zang, E. Dupuy, E. Peinke, P. Lalanne, J.-M. Gérard, and J. Claudon, Quantum dot spontaneous emission control in a ridge waveguide, Appl. Phys. Lett. 106, 041112 (2015).

[35] T. Hoehne, P. Schnauber, S. Rodt, S. Reitzenstein, and S. Burger, Numerical Investigation of Light Emission from Quantum Dots Embedded into On-Chip, LowIndex-Contrast Optical Waveguides, Phys. status solidi 256, 1800437 (2019).

[36] N. McLachlan, Theory and application of Mathieu functions (Clarendon, 1951).

[37] M. Abramowitz and I. A. Stegun, Handbook of Mathematical Functions with Formulas, Graphs, and Mathe- 
matical Tables, Vol. 55 (US Government Printing Office, 1970).

[38] U. S. N. B. of Standards Computation Laboratory, Tables Relating to Mathieu Functions: Characteristic Values, Coefficients, and Joining Factors, Applied mathematics series (U.S. Department of Commerce, National Bureau of Standards, 1967).

[39] J. Stratton, L. Chu, P. Morse, R. Hutner, and M. I. of Technology, Elliptic Cylinder and Spheroidal Wave Functions, Including Tables of Separation Constants and Coefficient (J. Wiley \& Sons, Incorporated, 1941).
[40] J. J. Stamnes and B. Spjelkavik, New method for computing eigenfunctions (mathieu functions) for scattering by elliptical cylinders, J. Opt. A-Pure Appl. Op. 4, 251 (1995).

[41] J. C. Gutiérrez-Vega, R. Rodrıguez-Dagnino, M. Meneses-Nava, and S. Chávez-Cerda, Mathieu functions, a visual approach, Am. J. Phys. 71, 233 (2003).

[42] A. Inayat-Hussain, Mathieu integral transforms, J. Math. Phys. 32, 669 (1991). 


\section{Open-geometry modal method based on transverse electric and transverse magnetic mode expansion for orthogonal curvilinear coordinates. \\ Supplementary Information}

\section{Scalar eigenproblem in generalized coordinates}

The scalar Helmholtz equation is given by

$$
\nabla^{2} \Psi(\mathbf{r})+\varepsilon_{r}\left(\mathbf{r}_{\perp}\right) k_{0}{ }^{2} \Psi(\mathbf{r})=0 .
$$

In the eigenmode expansion technique, we have uniformity in the axis of propagation such that $\varepsilon_{r}\left(\mathbf{r}_{\perp}\right)$ is independent of $z$. We thus use separation of variables

$$
\Psi\left(\mathbf{r}_{\perp}, z\right)=\Psi\left(\mathbf{r}_{\perp}\right) \exp (i \beta z),
$$

leading to the scalar eigenvalue equation

$$
\nabla_{\perp}^{2} \Psi\left(\mathbf{r}_{\perp}\right)+\varepsilon_{r}\left(\mathbf{r}_{\perp}\right) k_{0}^{2} \Psi\left(\mathbf{r}_{\perp}\right)=\beta^{2} \Psi\left(\mathbf{r}_{\perp}\right) .
$$

We now introduce the generalized coordinate transformation $(x, y) \rightarrow(u, v)$ in the plane normal to the propagation axis. The differential areas are related as

$$
l_{u} l_{v} d u d v=d x d y
$$

where $l_{u}(u, v)$ and $l_{v}(u, v)$ are the scale factors given by

$$
l_{u}=\left|\frac{d \mathbf{r}}{d u}\right| \quad l_{v}=\left|\frac{d \mathbf{r}}{d v}\right|
$$

The scalar wave equation for generalized curvilinear coordinates becomes

$$
\frac{1}{l_{u} l_{v}}\left(\frac{\partial}{\partial u} \frac{l_{v}}{l_{u}} \frac{\partial}{\partial u}+\frac{\partial}{\partial v} \frac{l_{u}}{l_{v}} \frac{\partial}{\partial v}\right) \Psi(u, v)+\varepsilon_{r}(u, v) k_{0}{ }^{2} \Psi(u, v)=\beta^{2} \Psi(u, v) .
$$

In the following we will often use the symbol $\Delta$

$$
\Delta=\frac{1}{l_{u} l_{v}}\left(\frac{\partial}{\partial u} \frac{l_{v}}{l_{u}} \frac{\partial}{\partial u}+\frac{\partial}{\partial v} \frac{l_{u}}{l_{v}} \frac{\partial}{\partial v}\right)
$$

to represent the Laplace operator in generalized coordinates. Additionally, we introduce the unit vectors $\mathbf{u}$ and $\mathbf{v}$ in generalized coordinates corresponding to the unit vectors $\mathbf{i}$ and $\mathbf{j}$ in Cartesian coordinates given by

$$
\mathbf{u}=\frac{\partial \mathbf{r}}{\partial u} \quad \mathbf{v}=\frac{\partial \mathbf{r}}{\partial v}
$$

For the particular case of cylindrical coordinates $(u, v)=(r, \phi)$, we have

$$
x=\cos (\phi) r \quad y=\sin (\phi) r
$$

and the scale factors become

$$
l_{r}=\left|\frac{d \mathbf{r}}{d r}\right|=\left|\frac{d(\mathbf{i} x+\mathbf{j} y)}{d r}\right|=\left|\frac{d(\mathbf{i} r \cos \phi+\mathbf{j} r \sin \phi)}{d r}\right|=|(\mathbf{i} \cos \phi+\mathbf{j} \sin \phi)|=1
$$




$$
l_{\phi}=\left|\frac{d \mathbf{r}}{d \phi}\right|=\left|\frac{d(\mathbf{i} x+\mathbf{j} y)}{d \phi}\right|=\left|\frac{d(\mathbf{i} r \cos \phi+\mathbf{j} r \sin \phi)}{d \phi}\right|=|(-\mathbf{i} r \sin \phi+\mathbf{j} r \cos \phi)|=r
$$

\subsection{Basis function}

In this Supplementary Information, we will assume that we can use separation of variables to write the solution to (6) for the empty geometry $\varepsilon_{r}(u, v)=1$ as

$$
\Psi_{q}(u, v)=U_{q}(u) V_{q}(v),
$$

where the subindex $q$ is an index of a particular solution. The general case for a non-separable basis function can be recovered by performing the substitution $U_{q}(u) V_{q}(v) \rightarrow \Psi_{q}(u, v)$. In this work, we consider geometries and coordinate systems (rotationally symmetric, elliptical) with $\varepsilon_{r}(u)$ independent of $v$, and where $V$ is a periodic function with period $2 \pi$ such that $U$ and $V$ become radial and angular basis functions, respectively.

We now define the in-plane $k$ vector amplitude $k_{\perp, q}$ for the solution of index $q$ by $k_{\perp, q}^{2}=k_{0}^{2}-\beta_{q}^{2}$. From (6) we obtain

$$
\Delta U_{q}(u) V_{q}(v)=-k_{\perp, q}^{2} U_{q}(u) V_{q}(v)
$$

for the empty geometry.

The functions (12) will be used to construct our vectorial basis modes, and the in-plane $k$ vector amplitudes $k_{\perp, q}$ should be chosen such that the boundary conditions are respected. We will return to the discussion of the boundary conditions once we have defined our vectorial basis modes in the next section.

In the first part of this Supplementary Information, we consider a closed geometry, where the computational domain is contained in the intervals $0 \leq u \leq u_{\max }$ and $0 \leq v \leq 2 \pi$. The open geometry formulation is discussed subsequently in Section 4 . We then require that our basis functions are normalized according to

$$
\int_{u=0, v=0}^{u=u_{\max }, v=2 \pi} U_{q^{\prime}}(u) V_{q^{\prime}}(v) U_{q}(u) V_{q}(v) l_{u} l_{v} d u d v=\delta_{q^{\prime} q} .
$$

Once suitable functions $U$ and $V$ have been identified, the normalization can be established by first computing the integral

$$
\int_{u=0, v=0}^{u=u_{\max }, v=v_{\max }} U_{q^{\prime}}(u) V_{q^{\prime}}(v) U_{q}(u) V_{q}(v) l_{u} l_{v} d u d v=N_{q} .
$$

and then performing the replacement $U_{q}(u) V_{q}(v) \rightarrow U_{q}(u) V_{q}(v) / \sqrt{N_{q}}$.

\subsection{Integration by parts}

We will use integration by parts extensively on three types of integrals in the subsequent calculations of the operator matrix elements in Section 3.1. In these derivations, we always assume that the boundary terms disappear. The first type of integral is of the form 


$$
\begin{aligned}
& \int_{v=0}^{v=2 \pi}\left(\frac{l_{u}}{l_{v}} \frac{\partial}{\partial v} U_{q^{\prime}}(u) V_{q^{\prime}}(v)\right)\left[\frac{\partial}{\partial v} U_{q}(u) V_{q}(v)\right] d v \\
& =\left[\left(\frac{l_{u}}{l_{v}} \frac{\partial}{\partial v} U_{q^{\prime}}(u) V_{q^{\prime}}(v)\right) U_{q}(u) V_{q}(v)\right]_{v=0}^{v=2 \pi}-\int_{v=0}^{v=2 \pi}\left(\frac{\partial}{\partial v} \frac{l_{u}}{l_{v}} \frac{\partial}{\partial v} U_{q^{\prime}}(u) V_{q^{\prime}}(v)\right)\left[U_{q}(u) V_{q}(v)\right] d u
\end{aligned}
$$

where the boundary term disappears due to periodicity of the angular basis function $V_{q}$. The second type of integral is

$$
\begin{aligned}
& \int_{u=0}^{u=u_{\max }}\left(\frac{l_{v}}{l_{u}} \frac{\partial}{\partial u} U_{q^{\prime}}(u) V_{q^{\prime}}(v)\right)\left[\frac{\partial}{\partial u} U_{q}(u) V_{q}(v)\right] d u \\
& =\left[\left(\frac{l_{v}}{l_{u}} \frac{\partial}{\partial u} U_{q^{\prime}}(u) V_{q^{\prime}}(v)\right) U_{q}(u) V_{q}(v)\right]_{u=0}^{u=u_{\max }}-\int_{u=0}^{u=u_{\max }}\left(\frac{\partial}{\partial u} \frac{l_{v}}{l_{u}} \frac{\partial}{\partial u} U_{q^{\prime}}(u) V_{q^{\prime}}(v)\right)\left[U_{q}(u) V_{q}(v)\right] d u
\end{aligned}
$$

where $u_{\max }$ takes either a finite value for a closed geometry boundary condition or is infinity for the open geometry formulation. Here, the boundary term in the second line of Eq. (17) does not automatically disappear, and special attention needs to be paid when choosing the basis functions for the specific geometry to verify that the boundary term is indeed zero. Finally, the third type of integral is

$$
\begin{aligned}
& \int_{u=0, v=0}^{u=u_{\max }, v=2 \pi}\left(\left[\begin{array}{ll}
\frac{\partial}{\partial u} & \frac{\partial}{\partial v}
\end{array}\right] U_{q^{\prime}, \mathrm{TX}}(u) V_{q^{\prime}, \mathrm{TX}}(v)\right)\left[\begin{array}{c}
\frac{\partial}{\partial v} \\
-\frac{\partial}{\partial u}
\end{array}\right] U_{q, \mathrm{TY}}(u) V_{q, \mathrm{TY}}(v) d u d v \\
& =\int_{v=0}^{v=2 \pi}\left[U_{q^{\prime}, \mathrm{TX}}(u) V_{q^{\prime}, \mathrm{TX}}(v) U_{q, \mathrm{TY}}(u) \frac{\partial}{\partial v} V_{q, \mathrm{TY}}(v)\right]_{u=0}^{u=u_{\max }} d v \\
& -\int_{u=0}^{u=u_{\max }}\left[U_{q^{\prime}, \mathrm{TX}}(u) V_{q^{\prime}, \mathrm{TX}}(v) \frac{\partial}{\partial u} U_{q, \mathrm{TY}}(u) V_{q, \mathrm{TY}}(v)\right]_{v=0}^{v=2 \pi} d u
\end{aligned}
$$

where TX and TY refer to polarizations of the TM and TE modes discussed later. The second boundary term on the RHS of (18) again disappears due to periodicity of $V_{q}$, however again special attention needs to be paid to verify that the first boundary term disappears. We will verify this in Sections 4, 5.1 and 6.1 for both the rotationally symmetric and the elliptical geometry.

The integration by parts will in some cases lead to an integral of the form

$$
\int U_{q^{\prime}}(u)\left(\frac{\partial}{\partial u} \varepsilon_{r}\right) \frac{l_{v}}{l_{u}}\left(\frac{\partial}{\partial u} U_{q}(u)\right) d u
$$

involving the derivative of $\varepsilon_{r}(u)$ with respect to $u$. In this work, we consider geometries with a piece-wise constant dielectric constant along $u$. Thus only the discontinuities of the dielectric constant will contribute to the integral (19), which can simply be written as

$$
\int U_{q^{\prime}}(u)\left(\frac{\partial}{\partial u} \varepsilon_{r}\right) \frac{l_{v}}{l_{u}}\left(\frac{\partial}{\partial u} U_{q}(u)\right) d u=\sum_{j} U_{q^{\prime}}\left(u_{j}\right)\left(\varepsilon_{r, j+}-\varepsilon_{r, j-}\right) \frac{l_{v}}{l_{u}}\left(\frac{\partial}{\partial u} U_{q}\left(u_{j}\right)\right),
$$

where $u_{j}$ are the positions of the discontinuities and $\varepsilon_{r, j+}-\varepsilon_{r, j-}$ is the difference between the dielectric constant after and before the discontinuity at the position $u_{j}$.

\section{Vectorial eigenproblem in generalized coordinates}

The curl and divergence operators in generalized coordinates $(u, v, z)$ can be written as 


$$
\begin{gathered}
\nabla \times \mathbf{A}=\left(\frac{1}{l_{v}} \frac{\partial A_{z}}{\partial v}-\frac{\partial A_{v}}{\partial z}\right) \mathbf{u}+\left(\frac{\partial A_{u}}{\partial z}-\frac{1}{l_{u}} \frac{\partial A_{z}}{\partial u}\right) \mathbf{v}+\frac{1}{l_{u} l_{v}}\left(\frac{\partial\left(l_{v} A_{v}\right)}{\partial u}-\frac{\partial\left(l_{u} A_{u}\right)}{\partial v}\right) \mathbf{z} \\
\nabla \cdot \mathbf{A}=\frac{1}{l_{u} l_{v}}\left(\frac{\partial\left(l_{v} A_{u}\right)}{\partial u}+\frac{\partial\left(l_{u} A_{v}\right)}{\partial v}\right)+\frac{\partial A_{z}}{\partial z} .
\end{gathered}
$$

For a fixed frequency $\omega_{0}$ the Maxwell equations can be written as:

$$
\begin{gathered}
\nabla \times \mathbf{E}=i \omega_{0} \mu_{0} \mathbf{H} \\
\nabla \times \mathbf{H}=-i \omega_{0} \varepsilon(u) \mathbf{E} .
\end{gathered}
$$

We will also need the relation between the electric field and the displacement field:

$$
\mathbf{D}=\varepsilon(u) \mathbf{E} .
$$

While the numerical problem is usually formulated simply in terms of the $\mathbf{E}$ and $\mathbf{H}$ fields, the discontinuity of the $E_{u}$ field at interfaces is poorly described using a series expansion approach. For this reason, it is convenient to compute also the displacement field, since this enables us to plot the $E_{u}$ component using the continuous $D_{u}$ component as $E_{u}=D_{u} / \varepsilon(u)$ leading to an improved representation of the discontinuous $E_{u}$ component.

As in the scalar case, we write the vectorial fields in the form

$$
\begin{aligned}
\mathbf{E}(u, v, z) & =\mathbf{e}(u, v) \exp (i \beta z) \\
\mathbf{H}(u, v, z) & =\mathbf{h}(u, v) \exp (i \beta z) \\
\mathbf{D}(u, v, z) & =\mathbf{d}(u, v) \exp (i \beta z) .
\end{aligned}
$$

\subsection{Basis modes}

We start by identifying the basis modes which subsequently will be used as an expansion basis for our eigenmodes. These will be defined as the solutions to the empty geometry, and for now we thus set $\varepsilon(u)=\varepsilon_{0}$.

Using the forms (26)-(27), Maxwell's equations (23)-(24) in generalized coordinates are then

$$
\begin{gathered}
i \omega_{0} \mu_{0} h_{u}=\frac{1}{l_{v}} \frac{\partial e_{z}}{\partial v}-i \beta e_{v} \\
i \omega_{0} \mu_{0} h_{v}=i \beta e_{u}-\frac{1}{l_{u}} \frac{\partial e_{z}}{\partial u} \\
i \omega_{0} \mu_{0} h_{z}=\frac{1}{l_{u} l_{v}}\left(\frac{\partial\left(l_{v} e_{v}\right)}{\partial u}-\frac{\partial\left(l_{u} e_{u}\right)}{\partial v}\right) \\
-i \omega_{0} \varepsilon_{0} e_{u}=\frac{1}{l_{v}} \frac{\partial h_{z}}{\partial v}-i \beta h_{v} \\
-i \omega_{0} \varepsilon_{0} e_{v}=i \beta h_{u}-\frac{1}{l_{u}} \frac{\partial h_{z}}{\partial u} \\
-i \omega_{0} \varepsilon_{0} e_{z}=\frac{1}{l_{u} l_{v}}\left(\frac{\partial\left(l_{v} h_{v}\right)}{\partial u}-\frac{\partial\left(l_{u} h_{u}\right)}{\partial v}\right) .
\end{gathered}
$$

We would now like to express the lateral components $e_{u}, e_{v}, h_{u}$, and $h_{v}$ in terms of $e_{z}$ and $h_{z}$. This can be done by combining the equations above and leads to

$$
e_{u}=i \frac{\beta}{k_{\perp}^{2} l_{u}} \frac{\partial e_{z}}{\partial u}+i \omega_{0} \mu_{0} \frac{1}{k_{\perp}^{2} l_{v}} \frac{\partial h_{z}}{\partial v}
$$




$$
\begin{aligned}
& e_{v}=i \frac{\beta}{k_{\perp}^{2} l_{v}} \frac{\partial e_{z}}{\partial v}-i \omega_{0} \mu_{0} \frac{1}{k_{\perp}^{2} l_{u}} \frac{\partial h_{z}}{\partial u} \\
& h_{u}=-i \omega_{0} \varepsilon_{0} \frac{1}{k_{\perp}^{2} l_{v}} \frac{\partial e_{z}}{\partial v}+i \frac{\beta}{k_{\perp}^{2} l_{u}} \frac{\partial h_{z}}{\partial u} \\
& h_{v}=i \omega_{0} \varepsilon_{0} \frac{1}{k_{\perp}^{2} l_{u}} \frac{\partial e_{z}}{\partial u}+i \frac{\beta}{k_{\perp}^{2} l_{v}} \frac{\partial h_{z}}{\partial v}
\end{aligned}
$$

where we have introduced the in-plane $k$ vector amplitude $k_{\perp}^{2}=k_{0}^{2}-\beta^{2}$ and used that $\varepsilon_{0} \mu_{0}=1 / c^{2}$. In the following we will generally use the scaling

$$
\begin{gathered}
\mathbf{H} \omega_{0} \mu_{0}=\mathbf{H}^{\prime} \\
\frac{\mathbf{D}}{\varepsilon_{0}}=\varepsilon_{r}(u, v) \mathbf{E}=\mathbf{D}^{\prime}
\end{gathered}
$$

to simplify the notation. With this notation, Eqs. (35)-(38) become

$$
\begin{gathered}
e_{u}=i \frac{\beta}{k_{\perp}^{2} l_{u}} \frac{\partial e_{z}}{\partial u}+i \frac{1}{k_{\perp}^{2} l_{v}} \frac{\partial h_{z}^{\prime}}{\partial v} \\
e_{v}=i \frac{\beta}{k_{\perp}^{2} l_{v}} \frac{\partial e_{z}}{\partial v}-i \frac{1}{k_{\perp}^{2} l_{u}} \frac{\partial h_{z}^{\prime}}{\partial u} \\
h_{u}^{\prime}=-i \frac{k_{0}^{2}}{k_{\perp}^{2} l_{v}} \frac{\partial e_{z}}{\partial v}+i \frac{\beta}{k_{\perp}^{2} l_{u}} \frac{\partial h_{z}^{\prime}}{\partial u} \\
h_{v}^{\prime}=i \frac{k_{0}^{2}}{k_{\perp}^{2} l_{u}} \frac{\partial e_{z}}{\partial u}+i \frac{\beta}{k_{\perp}^{2} l_{v}} \frac{\partial h_{z}^{\prime}}{\partial v},
\end{gathered}
$$

and in the following we will skip the " notation for the magnetic and displacement fields for simplicity.

Inspection of Eqs. (41)-(44) reveals that we can naturally define two sets of basis modes by setting either $e_{z}=0$ or $h_{z}=0$. We refer to the first set of modes as transverse electric (TE) and the second set of modes as transverse magnetic (TM).

Our empty geometry solutions are then given from the relations

$$
\begin{gathered}
e_{u}=\frac{1}{k_{\perp} l_{u}} \frac{\partial}{\partial u} U_{q, \mathrm{TM}}(u) V_{q, \mathrm{TM}}(v) \quad e_{v}=\frac{1}{k_{\perp} l_{v}} \frac{\partial}{\partial v} U_{q, \mathrm{TM}}(u) V_{q, \mathrm{TM}}(v) \quad e_{z}=\frac{k_{\perp}}{i \beta} U_{q, \mathrm{TM}}(u) V_{q, \mathrm{TM}}(v) \quad \text { (TM) } \\
h_{u}=-\frac{k_{0}^{2}}{k_{\perp} \beta} \frac{1}{l_{v}} \frac{\partial}{\partial v} U_{q, \mathrm{TM}}(u) V_{q, \mathrm{TM}}(v) \quad h_{v}=\frac{k_{0}^{2}}{k_{\perp} \beta} \frac{1}{l_{u}} \frac{\partial}{\partial u} U_{q, \mathrm{TM}}(u) V_{q, \mathrm{TM}}(v) h_{z}=0 \quad \text { (TM) } \\
e_{u}=\frac{1}{k_{\perp} \beta} \frac{1}{l_{v}} \frac{\partial}{\partial v} U_{q, \mathrm{TE}}(u) V_{q, \mathrm{TE}}(v) \quad e_{v}=-\frac{1}{k_{\perp} \beta} \frac{1}{l_{u}} \frac{\partial}{\partial u} U_{q, \mathrm{TE}}(u) V_{q, \mathrm{TE}}(v) e_{z}=0 \quad \text { (TE) } \\
h_{u}=\frac{1}{k_{\perp} l_{u}} \frac{\partial}{\partial u} U_{q, \mathrm{TE}}(u)_{q, \mathrm{TE}} V(v) \quad h_{v}=\frac{1}{k_{\perp} l_{v}} \frac{\partial}{\partial v} U_{q, \mathrm{TE}}(u) V_{q, \mathrm{TE}}(v) \quad h_{z}=\frac{k_{\perp}}{i \beta} U_{q, \mathrm{TE}}(u) V_{q, \mathrm{TE}}(v) \quad \text { (TE) }
\end{gathered}
$$

Since the scaled displacement field (40) is identical the electric field in the empty geometry $\left(\varepsilon_{r}=1\right)$, the empty geometry solutions for the displacement field are identical to those (45),(47) for the electric fields.

We will now define basis modes $\mathbf{f}(u, v)$ and $\mathbf{g}(u, v)$ for the planar $(u, v)$ components of the electric (and displacement) and the magnetic fields as the TE and TM solutions to the empty geometry 


$$
\begin{aligned}
& \mathbf{f}_{q, \mathrm{TM}}=\left[\begin{array}{ll}
f_{u, q, \mathrm{TM}} & f_{v, q, \mathrm{TM}}
\end{array}\right]^{T}=\left[\frac{1}{k_{\perp, q, \mathrm{TM}} l_{u}} \frac{\partial}{\partial u} U_{q, \mathrm{TM}}(u) V_{q, \mathrm{TM}}(v) \frac{1}{k_{\perp, q, \mathrm{TM}} l_{v}} \frac{\partial}{\partial v} U_{q, \mathrm{TM}}(u) V_{q, \mathrm{TM}}(v)\right]^{T} \\
& \mathbf{g}_{q, \mathrm{TM}}=\left[\begin{array}{ll}
g_{u, q, \mathrm{TM}} & g_{v, q, \mathrm{TM}}
\end{array}\right]^{T}=\left[-\frac{1}{k_{\perp, q, \mathrm{TM}} l_{v}} \frac{\partial}{\partial v} U_{q, \mathrm{TM}}(u) V_{q, \mathrm{TM}}(v) \frac{1}{k_{\perp, q, \mathrm{TM}} l} \frac{\partial}{\partial u} U_{q, \mathrm{TM}}(u) V_{q, \mathrm{TM}}(v)\right]^{T} \\
& \mathbf{f}_{q, \mathrm{TE}}=\left[\begin{array}{ll}
f_{u, q, \mathrm{TE}} & f_{v, q, \mathrm{TE}}
\end{array}\right]^{T}=\left[\frac{1}{k_{\perp, q, \mathrm{TE}} l_{u}} \frac{\partial}{\partial u} U_{q, \mathrm{TE}}(u) V_{q, \mathrm{TE}}(v) \frac{1}{k_{\perp, q, \mathrm{TE}} l} \frac{\partial}{\partial v} U_{q, \mathrm{TE}}(u) V_{q, \mathrm{TE}}(v)\right]^{T} \\
& \mathbf{g}_{q, \mathrm{TE}}=\left[\begin{array}{ll}
g_{u, q, \mathrm{TE}} & g_{v, q, \mathrm{TE}}
\end{array}\right]^{T}=\left[\frac{1}{k_{\perp, q, \mathrm{TE}} l_{v}} \frac{\partial}{\partial v} U_{q, \mathrm{TE}}(u) V_{q, \mathrm{TE}}(v)-\frac{1}{k_{\perp, q, \mathrm{TE}} l_{u}} \frac{\partial}{\partial u} U_{q, \mathrm{TE}}(u) V_{q, \mathrm{TE}}(v)\right]^{T},
\end{aligned}
$$

where the superindex ${ }^{T}$ indicates matrix transposition. We note that different basis modes for TM or TE are needed due to the boundary condition described below. Additionally, we introduce the basis modes $\Psi_{q, \mathrm{TX}}(u, v)$ for the $z$ components of the fields, which are simply identical to our basis functions

$$
\Psi_{q, \mathrm{TX}}(u, v)=U_{q, \mathrm{TX}}(u) V_{q, \mathrm{TX}}(v),
$$

The components of the electromagnetic fields of the eigenmode $j$ are then expanded on the basis modes as

$$
\begin{aligned}
& \mathbf{e}_{\perp, j}(u, v)= {\left[\begin{array}{l}
e_{u, j}(u, v) \\
e_{v, j}(u, v)
\end{array}\right]=\sum_{q} c_{\mathrm{TM}, q j}^{E_{\perp}} \mathbf{f}_{q, \mathrm{TM}}(u, v)+\sum_{q} c_{\mathrm{TE}, q j}^{E_{\perp}} \mathbf{g}_{q, \mathrm{TE}}(u, v) } \\
& \mathbf{h}_{\perp, j}(u, v)=\left[\begin{array}{l}
h_{u, j}(u, v) \\
h_{v, j}(u, v)
\end{array}\right]=\sum_{q} c_{\mathrm{TM}, q j}^{H_{\perp}} \mathbf{g}_{q, \mathrm{TM}}(u, v)+\sum_{q} c_{\mathrm{TE}, q j}^{H_{\perp}} \mathbf{f}_{q, \mathrm{TE}}(u, v) \\
& \mathbf{d}_{\perp, j}(u, v)=\left[\begin{array}{c}
d_{u, j}(u, v) \\
d_{v, j}(u, v)
\end{array}\right]=\sum_{q} c_{\mathrm{TM}, q j}^{D_{\perp}} \mathbf{f}_{q, \mathrm{TM}}(u, v)+\sum_{q} c_{\mathrm{TE}, q j}^{D_{\perp}} \mathbf{g}_{q, \mathrm{TE}}(u, v) \\
& e_{z, j}(u, v)=\sum_{q} c_{q j}^{E_{z}} \Psi_{q, \mathrm{TM}}(u, v) \\
& h_{z, j}(u, v)=\sum_{q} c_{q j}^{H_{z}} \Psi_{q, \mathrm{TE}}(u, v),
\end{aligned}
$$

and our task will be to compute the expansion vectors $\overline{c_{\mathrm{TE}, j}^{E_{\perp}}}, \overline{c_{\mathrm{TM}, j}^{E_{\perp}}}, \overline{c_{j}^{E_{z}}}, \overline{c_{\mathrm{TE}, j}^{H_{\perp}}}, \overline{c_{\mathrm{TM}, j}^{H_{\perp}}}, \overline{c_{j}^{H_{z}}}, \overline{c_{\mathrm{TE}, j}^{D_{\perp}}}$, $\overline{c_{\mathrm{TM}, j}^{D_{\perp}}}$ describing the eigenmode $j$.

\subsection{Boundary conditions for the closed geometry}

We initially discuss the boundary conditions of the closed geometry where $0 \leq u \leq u_{\max }$, and the open geometry formulation $0 \leq u \leq \infty$ will be presented in Section 4 . For the closed geometry we assume a perfect electric conductor at the boundary $u_{\max }$, such that the tangential components of the electric field are 0 at $u=u_{\max }$. For the TM modes, the condition is given by

$$
e_{z, q}\left(u_{\max }, v\right)=\frac{k_{\perp, q, \mathrm{TM}}}{i \beta} U_{q, \mathrm{TM}}\left(u_{\max }\right) V_{q, \mathrm{TM}}(v)=U_{q, \mathrm{TM}}\left(u_{\text {max }}\right)=0
$$

since $V_{q} \neq 0$. The TE modes have no $e_{z}$ component, so instead we require that

$$
e_{v, q}\left(u_{\max }, v\right)=-\frac{1}{k_{\perp} \beta} \frac{1}{l_{u}} \frac{\partial}{\partial u} U_{q, \mathrm{TE}}\left(u_{\max }\right) V_{q, \mathrm{TE}}(v)=\frac{\partial}{\partial u} U_{q, \mathrm{TE}}\left(u_{\max }\right)=0 .
$$


The values of the in-plane $k$ vector $k_{\perp, q, \mathrm{TX}}$ describe the propagation of the radial basis functions $U_{q, \mathrm{TX}}(u)$ and should be chosen such that the conditions (59)-(60) are respected. We notice that with the boundary conditions (59)-(60) the $u=u_{\max }$ contribution to the boundary terms in Eq. (17) and (18) disappears. The $u=0$ term will be discussed in Sections 5.1 and 6.1.

\subsection{Orthonormalization of basis modes}

It is convenient to introduce the following bra-ket notation for vector function products

$$
\begin{aligned}
& \langle\mathbf{a} \mid \mathbf{b}\rangle=\left[\begin{array}{ll}
a_{u} & a_{v}
\end{array}\right]\left[\begin{array}{l}
b_{u} \\
b_{v}
\end{array}\right] \\
& =\int_{u=0, v=0}^{u=u_{\max }, v=2 \pi}\left[\begin{array}{ll}
a_{u}(u, v) & a_{v}(u, v)
\end{array}\right]\left[\begin{array}{l}
b_{u}(u, v) \\
b_{v}(u, v)
\end{array}\right] l_{u} l_{v} d u d v, \\
& =\int_{u=0, v=0}^{u=u_{\max }, v=2 \pi}\left(a_{u}(u, v) b_{u}(u, v)+a_{v}(u, v) b_{v}(u, v)\right) l_{u} l_{v} d u d v
\end{aligned}
$$

such that products of vector functions includes an integration over $u$ and $v$. This notation can also be defined for scalar function products as

$$
\langle a \mid b\rangle=\int_{u=0, v=0}^{u=u_{\max }, v=2 \pi} a(u, v) b(u, v) l_{u} l_{v} d u d v .
$$

The orthonormalization condition for the basis modes is then given by

$$
\begin{aligned}
& \left\langle\mathbf{f}_{q^{\prime}, \mathrm{TX}} \mid \mathbf{f}_{q, \mathrm{TX}}\right\rangle=\left\langle\mathbf{g}_{q^{\prime}, \mathrm{TX}} \mid \mathbf{g}_{q, \mathrm{TX}}\right\rangle=\frac{1}{k_{\perp, q} k_{\perp, q}} \int\left[\left(\frac{\partial}{\partial u} U_{q^{\prime}, \mathrm{TX}} V_{q^{\prime}, \mathrm{TX}}\right) \frac{l_{v}}{l_{u}}\left(\frac{\partial}{\partial u} U_{q, \mathrm{TX}} V_{q, \mathrm{TX}}\right)+\left(\frac{\partial}{\partial v} U_{q^{\prime}, \mathrm{TX}} V_{q^{\prime}, \mathrm{TX}}\right) \frac{l_{u}}{l_{v}}\left(\frac{\partial}{\partial v} U_{q, \mathrm{TX}} V_{q, \mathrm{TX}}\right)\right] d u d v \\
& =-\frac{1}{k_{\perp, q^{\prime}} k_{\perp, q}} \int\left[\left(\frac{\partial}{\partial u} \frac{l_{v}}{l_{u}} \frac{\partial}{\partial u} U_{q^{\prime}, \mathrm{TX}} V_{q^{\prime}, \mathrm{TX}}\right) U_{q, \mathrm{TX}} V_{q, \mathrm{TX}}+\left(\frac{\partial}{\partial v} \frac{l_{u}}{l_{v}} \frac{\partial}{\partial v} U_{q^{\prime}, \mathrm{TX}} V_{q^{\prime}, \mathrm{TX}}\right) U_{q, \mathrm{TX}} V_{q, \mathrm{TX}}\right] d u d v \\
& =-\frac{1}{k_{\perp, q^{\prime}} k_{\perp, q}} \int l_{v} l_{u}\left(\Delta U_{q^{\prime}, \mathrm{TX}} V_{q^{\prime}, \mathrm{TX}}\right) U_{q, \mathrm{TX}} V_{q, \mathrm{TX}} d u d v=\frac{k_{\perp, q^{\prime}}}{k_{\perp, q}} \int l_{v} l_{u} U_{q^{\prime}, \mathrm{TX}} V_{q^{\prime}, \mathrm{TX}} U_{q, \mathrm{TX}} V_{q, \mathrm{TX}} d u d v=\delta_{q^{\prime} q}
\end{aligned}
$$

where we used integration by parts Eqs. (16)-(17) in the second step. Additionally, we have

$$
\begin{aligned}
& \left\langle\mathbf{f}_{q^{\prime}, \mathrm{TX}} \mid \mathbf{g}_{q, \mathrm{TY}}\right\rangle=\left\langle\mathbf{g}_{q, \mathrm{TX}} \mid \mathbf{f}_{q^{\prime}, \mathrm{TY}}\right\rangle \\
& =\frac{1}{k_{\perp, q^{\prime}} k_{\perp, q}} \int\left[\left(\frac{\partial}{\partial u} U_{q^{\prime}, \mathrm{TX}} V_{q^{\prime}, \mathrm{TX}}\right)\left(\frac{\partial}{\partial v} U_{q, \mathrm{TY}} V_{q, \mathrm{TY}}\right)-\left(\frac{\partial}{\partial v} U_{q^{\prime}, \mathrm{TX}} V_{q^{\prime}, \mathrm{TX}}\right)\left(\frac{\partial}{\partial u} U_{q, \mathrm{TY}} V_{q, \mathrm{TY}}\right)\right] d u d v, \\
& =-\frac{1}{k_{\perp, q^{\prime}} k_{\perp, q}} \int\left[\left(\frac{\partial}{\partial u} \frac{\partial}{\partial v} U_{q^{\prime}, \mathrm{TX}} V_{q^{\prime}, \mathrm{TX}}\right) U_{q, \mathrm{TY}} V_{q, \mathrm{TY}}-\left(\frac{\partial}{\partial v} \frac{\partial}{\partial u} U_{q^{\prime}, \mathrm{TX}} V_{q^{\prime}, \mathrm{TX}}\right) U_{q, \mathrm{TY}} V_{q, \mathrm{TY}}\right] d u d v=0
\end{aligned}
$$

where integration by parts Eq. (18) was employed in the second step.

\section{Operator matrices}

Let us recall that Maxwell's equations with the scaling (39) in the general case are given by

$$
\begin{gathered}
\nabla \times \mathbf{E}=i \mathbf{H} \\
\nabla \times \mathbf{H}=-i k_{0}^{2} \varepsilon_{r}(u) \mathbf{E} .
\end{gathered}
$$

By writing out the curl operators and using the forms (26)-(27), Eqs. (65)-(66) can be written as 


$$
\begin{gathered}
\left(\frac{1}{l_{v}} \frac{\partial}{\partial v} e_{z}-i \beta e_{v}\right) \mathbf{u}+\left(i \beta e_{u}-\frac{1}{l_{u}} \frac{\partial}{\partial u} e_{z}\right) \mathbf{v}+\frac{1}{l_{u} l_{v}}\left(\frac{\partial}{\partial u} l_{v} e_{v}-\frac{\partial}{\partial v} l_{u} e_{u}\right) \mathbf{z}=i \mathbf{h} \\
\left(\frac{1}{l_{v}} \frac{\partial}{\partial v} h_{z}-i \beta h_{v}\right) \mathbf{u}+\left(i \beta h_{u}-\frac{1}{l_{u}} \frac{\partial}{\partial u} h_{z}\right) \mathbf{v}+\frac{1}{l_{u} l_{v}}\left(\frac{\partial}{\partial u} l_{v} h_{v}-\frac{\partial}{\partial v} l_{u} h_{u}\right) \mathbf{z}=-i k_{0}^{2} \varepsilon_{r}(u) \mathbf{e} .
\end{gathered}
$$

Rearranging gives:

$$
\begin{gathered}
i\left(\frac{1}{l_{v}} \frac{\partial}{\partial v} e_{z}\right) \mathbf{u}-i\left(\frac{1}{l_{u}} \frac{\partial}{\partial u} e_{z}\right) \mathbf{v}+\frac{i}{l_{u} l_{v}}\left(\frac{\partial}{\partial u} l_{v} e_{v}-\frac{\partial}{\partial v} l_{u} e_{u}\right) \mathbf{z}+\mathbf{h}=-\beta e_{v} \mathbf{u}+\beta e_{u} \mathbf{v} \\
i\left(\frac{1}{l_{v}} \frac{\partial}{\partial v} h_{z}\right) \mathbf{u}-i\left(\frac{1}{l_{u}} \frac{\partial}{\partial u} h_{z}\right) \mathbf{v}+\frac{i}{l_{u} l_{v}}\left(\frac{\partial}{\partial u} l_{v} h_{v}-\frac{\partial}{\partial v} l_{u} h_{u}\right) \mathbf{z}-k_{0}^{2} \varepsilon_{r}(u) \mathbf{e}=-\beta h_{v} \mathbf{u}+\beta h_{u} \mathbf{v}
\end{gathered}
$$

In matrix form this is:

$$
\left[\begin{array}{cccccc}
0 & 0 & i \frac{1}{l_{v}} \frac{\partial}{\partial v} & 1 & 0 & 0 \\
0 & 0 & -i \frac{1}{l_{u}} \frac{\partial}{\partial u} & 0 & 1 & 0 \\
-i \frac{1}{l_{u} l_{v}} \frac{\partial}{\partial v} l_{u} & i \frac{1}{l_{u} l_{v}} \frac{\partial}{\partial u} l_{v} & 0 & 0 & 0 & 1 \\
-k_{0}^{2} \varepsilon_{r} & 0 & 0 & 0 & 0 & i \frac{1}{l_{v}} \frac{\partial}{\partial v} \\
0 & -k_{0}^{2} \varepsilon_{r} & 0 & 0 & 0 & -i \frac{1}{l_{u}} \frac{\partial}{\partial u} \\
0 & 0 & -k_{0}^{2} \varepsilon_{r} & -i \frac{1}{l_{u} l_{v}} \frac{\partial}{\partial v} l_{u} & i \frac{1}{l_{u} l_{v}} \frac{\partial}{\partial u} l_{v} & 0
\end{array}\right]\left[\begin{array}{c}
e_{u} \\
e_{v} \\
e_{z} \\
h_{u} \\
h_{v} \\
h_{z}
\end{array}\right]=\beta\left[\begin{array}{cccccc}
0 & -1 & 0 & 0 & 0 & 0 \\
1 & 0 & 0 & 0 & 0 & 0 \\
0 & 0 & 0 & 0 & 0 & 0 \\
0 & 0 & 0 & 0 & -1 & 0 \\
0 & 0 & 0 & 1 & 0 & 0 \\
0 & 0 & 0 & 0 & 0 & 0
\end{array}\right]\left[\begin{array}{c}
e_{u} \\
e_{v} \\
e_{z} \\
h_{u} \\
h_{v} \\
h_{z}
\end{array}\right]
$$

This is modified slightly to make the RHS symmetric:

$$
\left[\begin{array}{cccccc}
0 & 0 & -i \frac{1}{l_{u}} \frac{\partial}{\partial u} & 0 & 1 & 0 \\
0 & 0 & -i \frac{1}{l_{v}} \frac{\partial}{\partial v} & -1 & 0 & 0 \\
-i \frac{1}{l_{u} l_{v}} \frac{\partial}{\partial v} l_{u} & i \frac{1}{l_{u} l_{v}} \frac{\partial}{\partial u} l_{v} & 0 & 0 & 0 & 1 \\
0 & -k_{0}^{2} \varepsilon_{r} & 0 & 0 & 0 & -i \frac{1}{l_{u}} \frac{\partial}{\partial u} \\
k_{0}^{2} \varepsilon_{r} & 0 & 0 & 0 & 0 & -i \frac{1}{l_{v}} \frac{\partial}{\partial v} \\
0 & 0 & -k_{0}^{2} \varepsilon_{r} & -i \frac{1}{l_{u} l_{v}} \frac{\partial}{\partial v} l_{u} & i \frac{1}{l_{u} l_{v}} \frac{\partial}{\partial u} l_{v} & 0
\end{array}\right]\left[\begin{array}{l}
e_{u} \\
e_{v} \\
e_{z} \\
u_{u} \\
h_{v} \\
h_{z}
\end{array}\right]=\beta\left[\begin{array}{llllll}
1 & 0 & 0 & 0 & 0 & 0 \\
0 & 1 & 0 & 0 & 0 & 0 \\
0 & 0 & 0 & 0 & 0 & 0 \\
0 & 0 & 0 & 1 & 0 & 0 \\
0 & 0 & 0 & 0 & 1 & 0 \\
0 & 0 & 0 & 0 & 0 & 0
\end{array}\right]\left[\begin{array}{c}
e_{u} \\
e_{v} \\
e_{z} \\
h_{u} \\
h_{v} \\
h_{z}
\end{array}\right]
$$

We can eliminate the $e_{z}$ and $h_{z}$ components using:

$$
\begin{gathered}
\frac{1}{l_{u} l_{v}} \frac{\partial}{\partial v} l_{u} e_{u}-\frac{1}{l_{u} l_{v}} \frac{\partial}{\partial u} l_{v} e_{v}=-i h_{z} \\
-\frac{1}{k_{0}^{2} \varepsilon_{r}} \frac{1}{l_{u} l_{v}} \frac{\partial}{\partial v} l_{u} h_{u}+\frac{1}{k_{0}^{2} \varepsilon_{r}} \frac{1}{l_{u} l_{v}} \frac{\partial}{\partial u} l_{v} h_{v}=-i e_{z} .
\end{gathered}
$$

This gives: 


$$
\begin{aligned}
& {\left[\begin{array}{cccc}
0 & 0 & -\frac{1}{l_{u}} \frac{\partial}{\partial u} \frac{1}{k_{0}^{2} \varepsilon_{r}} \frac{1}{l_{u} l_{v}} \frac{\partial}{\partial v} l_{u} & 1+\frac{1}{l_{u}} \frac{\partial}{\partial u} \frac{1}{k_{0}^{2} \varepsilon_{r}} \frac{1}{l_{u} l_{v}} \frac{\partial}{\partial u} l_{v} \\
0 & 0 & -1-\frac{1}{l_{v}} \frac{\partial}{\partial v} \frac{1}{k_{0}^{2} \varepsilon_{r}} \frac{1}{l_{u} l_{v}} \frac{\partial}{\partial v} l_{u} & \frac{1}{l_{v}} \frac{\partial}{\partial v} \frac{1}{k_{0}^{2} \varepsilon_{r}} \frac{1}{l_{u} l_{v}} \frac{\partial}{\partial u} l_{v} \\
\frac{1}{l_{u}} \frac{\partial}{\partial u} \frac{1}{l_{u} l_{v}} \frac{\partial}{\partial v} l_{u} & -k_{0}^{2} \varepsilon_{r}-\frac{1}{l_{u}} \frac{\partial}{\partial u} \frac{1}{l_{u} l_{v}} \frac{\partial}{\partial u} l_{v} & 0 & 0 \\
k_{0}^{2} \varepsilon_{r}+\frac{1}{l_{v}} \frac{\partial}{\partial v} \frac{1}{l_{u} l_{v}} \frac{\partial}{\partial v} l_{u} & -\frac{1}{l_{v}} \frac{\partial}{\partial v} \frac{1}{l_{u} l_{v}} \frac{\partial}{\partial u} l_{v} & 0 & 0
\end{array}\right]\left[\begin{array}{c}
e_{u} \\
e_{v} \\
h_{u} \\
h_{v}
\end{array}\right]} \\
& =\beta\left[\begin{array}{l}
e_{u} \\
e_{v} \\
h_{u} \\
h_{v}
\end{array}\right]
\end{aligned}
$$

We note that the diagonal elements coupling the electric and magnetic field components with themselves are all zero. The above can thus be written as

$$
\begin{gathered}
\hat{O}_{e h}\left[\begin{array}{l}
h_{u} \\
h_{v}
\end{array}\right]=\beta\left[\begin{array}{l}
e_{u} \\
e_{v}
\end{array}\right] \\
\hat{O}_{h e}\left[\begin{array}{l}
e_{u} \\
e_{v}
\end{array}\right]=\beta\left[\begin{array}{l}
h_{u} \\
h_{v}
\end{array}\right],
\end{gathered}
$$

where

$$
\begin{gathered}
\hat{O}_{e h}=\left[\begin{array}{cc}
-\frac{1}{l_{u}} \frac{\partial}{\partial u} \frac{1}{k_{0}^{2} \varepsilon_{r}} \frac{1}{l_{u} l_{v}} \frac{\partial}{\partial v} l_{u} & 1+\frac{1}{l_{u}} \frac{\partial}{\partial u} \frac{1}{k_{0}^{2} \varepsilon_{r}} \frac{1}{l_{u} l_{v}} \frac{\partial}{\partial u} l_{v} \\
-1-\frac{1}{l_{v}} \frac{\partial}{\partial v} \frac{1}{k_{0}^{2} \varepsilon_{r}} \frac{1}{l_{u} l_{v}} \frac{\partial}{\partial v} l_{u} & \frac{1}{l_{v}} \frac{\partial}{\partial v} \frac{1}{k_{0}^{2} \varepsilon_{r}} \frac{1}{l_{u} l_{v}} \frac{\partial}{\partial u} l_{v}
\end{array}\right] \\
\hat{O}_{h e}=\left[\begin{array}{cc}
\frac{1}{l_{u}} \frac{\partial}{\partial u} \frac{1}{l_{u} l_{v}} \frac{\partial}{\partial v} l_{u} & -k_{0}^{2} \varepsilon_{r}-\frac{1}{l_{u}} \frac{\partial}{\partial u} \frac{1}{l_{u} l_{v}} \frac{\partial}{\partial u} l_{v} \\
k_{0}^{2} \varepsilon_{r}+\frac{1}{l_{v}} \frac{\partial}{\partial v} \frac{1}{l_{u} l_{v}} \frac{\partial}{\partial v} l_{u} & -\frac{1}{l_{v}} \frac{\partial}{\partial v} \frac{1}{l_{u} l_{v}} \frac{\partial}{\partial u} l_{v}
\end{array}\right] .
\end{gathered}
$$

We now expand the in-plane electric and magnetic fields on our basis modes according to (54)-(55) and write the expansions in matrix notation as

$$
\begin{aligned}
& {\left[\begin{array}{l}
e_{u} \\
e_{v}
\end{array}\right]=\sum_{q} c_{\mathrm{TM}, q}^{E_{\perp}} \mathbf{f}_{q, \mathrm{TM}}(u, v)+\sum_{q} c_{\mathrm{TE}, q}^{E_{\perp}} \mathbf{g}_{q, \mathrm{TE}}(u, v)=\left[\begin{array}{ll}
\bar{f}_{u, \mathrm{TM}}^{T} & \bar{g}_{u, \mathrm{TE}}^{T} \\
\bar{f}_{v, \mathrm{TM}}^{T} & \bar{g}_{v, \mathrm{TE}}^{T}
\end{array}\right]\left[\begin{array}{c}
-E_{\perp} \\
c_{\mathrm{TM}} \\
-c_{\perp} \\
c_{\mathrm{TE}}
\end{array}\right]} \\
& {\left[\begin{array}{l}
h_{u} \\
h_{v}
\end{array}\right]=\sum_{q} c_{\mathrm{TM}, q}^{H_{\perp}} \mathbf{g}_{q, \mathrm{TM}}(u, v)+\sum_{q} c_{\mathrm{TE}, q}^{H_{\perp}} \mathbf{f}_{q, \mathrm{TE}}(u, v)=\left[\begin{array}{ll}
\bar{g}_{u, \mathrm{TM}}^{T} & \bar{f}_{u, \mathrm{TE}}^{T} \\
\bar{g}_{v, \mathrm{TM}}^{T} & \bar{g}_{v, \mathrm{TE}}^{T}
\end{array}\right]\left[\begin{array}{l}
\bar{H}_{\perp} \\
c_{\mathrm{TM}} \\
-H_{\perp} \\
c_{\mathrm{TE}}
\end{array}\right],}
\end{aligned}
$$

where $\bar{f}_{i, \mathrm{TX}}$ and $\bar{g}_{i, \mathrm{TX}}$ are column vectors containing the functions $f_{i, q, \mathrm{TX}}(u, v)$ and $g_{i, q, \mathrm{TX}}(u, v)$ in the column elements and $\bar{c}_{\mathrm{TX}}$ and $\bar{c}_{\mathrm{TX}}$ are column vectors containing the expansion coefficients $c_{\mathrm{q}, \mathrm{TX}}^{E_{\perp}}$ and $c_{q, \mathrm{TX}}^{H_{\perp}}$ in the column elements.

With this notation, the operator Eq. (76) can be written as 


$$
\hat{O}_{e h}\left[\begin{array}{ll}
\bar{g}_{u, \mathrm{TM}}^{T} & \bar{f}_{u, \mathrm{TE}}^{T} \\
\bar{g}_{v, \mathrm{TM}}^{T} & \bar{f}_{v, \mathrm{TE}}^{T}
\end{array}\right]\left[\begin{array}{l}
-H_{\perp} \\
\overline{\mathrm{TM}}_{\mathrm{TM}} \\
-H_{\perp} \\
\bar{c}_{\mathrm{TE}}
\end{array}\right]=\beta\left[\begin{array}{ll}
\bar{f}_{u, \mathrm{TM}}^{T} & \bar{g}_{u, \mathrm{TE}}^{T} \\
\bar{f}_{v, \mathrm{TM}}^{T} & \bar{g}_{v, \mathrm{TE}}^{T}
\end{array}\right]\left[\begin{array}{l}
-E_{\perp} \\
\overline{\mathrm{TM}} \\
\bar{c}_{\perp}
\end{array}\right]
$$

From the orthonormality condition (63), we have the identity

$$
\int\left[\begin{array}{ll}
\bar{f}_{u, \mathrm{TM}} & \bar{f}_{v, \mathrm{TM}} \\
\bar{g}_{u, \mathrm{TE}} & \bar{g}_{v, \mathrm{TE}}
\end{array}\right]\left[\begin{array}{ll}
\bar{f}_{u, \mathrm{TM}}^{T} & \bar{g}_{u, \mathrm{TE}}^{T} \\
\bar{f}_{v, \mathrm{TM}}^{T} & \bar{g}_{v, \mathrm{TE}}^{T}
\end{array}\right] d \mathbf{r}_{\perp}=\int\left[\begin{array}{ll}
\bar{g}_{u, \mathrm{TM}} & \bar{g}_{v, \mathrm{TM}} \\
\bar{f}_{u, \mathrm{TE}} & \bar{f}_{v, \mathrm{TE}}
\end{array}\right]\left[\begin{array}{ll}
\bar{g}_{u, \mathrm{TM}}^{T} & \bar{f}_{u, \mathrm{TE}}^{T} \\
\bar{g}_{v, \mathrm{TM}}^{T} & \bar{f}_{v, \mathrm{TE}}^{T}
\end{array}\right] d \mathbf{r}_{\perp}=\left[\begin{array}{ll}
\overline{\bar{I}} & \overline{0} \\
\overline{0} & \overline{\bar{I}}
\end{array}\right],
$$

where $\overline{\bar{I}}$ and $\overline{\overline{0}}$ are the identity and zero matrices respectively. Multiplying (82) with $\int\left[\begin{array}{ll}\bar{f}_{u, \mathrm{TM}} & \bar{f}_{v, \mathrm{TM}} \\ \bar{g}_{u, \mathrm{TE}} & \bar{g}_{v, \mathrm{TE}}\end{array}\right] d \mathbf{r}_{\perp}$ then gives

$$
\int\left[\begin{array}{ll}
\bar{f}_{u, \mathrm{TM}} & \bar{f}_{v, \mathrm{TM}} \\
\bar{g}_{u, \mathrm{TE}} & \bar{g}_{v, \mathrm{TE}}
\end{array}\right] \hat{O}_{e h}\left[\begin{array}{ll}
\bar{g}_{u, \mathrm{TM}}^{T} & \bar{f}_{u, \mathrm{TE}}^{T} \\
\bar{g}_{v, \mathrm{TM}}^{T} & \bar{f}_{v, \mathrm{TE}}^{T}
\end{array}\right]\left[\begin{array}{l}
-H_{\perp} \\
\bar{c}_{\mathrm{TM}} \\
\bar{c}_{\perp}
\end{array}\right] d \mathbf{r}_{\perp}=\beta\left[\begin{array}{l}
-E_{\perp} \\
-E_{\perp} \\
\bar{c}_{\mathrm{TE}}
\end{array}\right]
$$

and we can then define an operator matrix $\overline{\bar{O}}_{e h}$ as

$$
\overline{\bar{O}}_{e h}=\left[\begin{array}{ll}
\overline{\bar{O}}_{e h}^{\mathrm{TMTM}} & \overline{\bar{O}}_{e h}^{\mathrm{TMTE}} \\
\overline{\bar{O}}_{e h}^{\mathrm{TETM}} & \overline{\bar{O}}_{e h}^{\mathrm{TETE}}
\end{array}\right]=\left[\left[\begin{array}{ll}
\bar{f}_{u, \mathrm{TM}} & \bar{f}_{v, \mathrm{TM}} \\
\bar{g}_{u, \mathrm{TE}} & \bar{g}_{v, \mathrm{TE}}
\end{array}\right] \hat{O}_{e h}\left[\begin{array}{ll}
\bar{g}_{u, \mathrm{TM}}^{T} & \bar{f}_{u, \mathrm{TE}}^{T} \\
\bar{g}_{v, \mathrm{TM}}^{T} & \bar{f}_{v, \mathrm{TE}}^{T}
\end{array}\right] d \mathbf{r}_{\perp},\right.
$$

such that

$$
\overline{\bar{O}}_{e h}\left[\begin{array}{c}
-H_{\perp} \\
c_{\mathrm{TM}} \\
-H_{\perp} \\
c_{\mathrm{TE}}
\end{array}\right]=\beta\left[\begin{array}{c}
-E_{\perp} \\
c_{\mathrm{TM}} \\
-E_{\perp} \\
c_{\mathrm{TE}}
\end{array}\right]
$$

For example, the element $\left(q, q^{\prime}\right)$ of the matrix $\overline{\bar{O}}_{e h}^{\mathrm{TMTM}}$ is labelled $O_{e h, q^{\prime} q}^{\mathrm{TMTM}}$ and is given by

$$
O_{e h, q^{\prime} q}^{\mathrm{TMTM}}=\int\left[\begin{array}{ll}
f_{u, q^{\prime}, \mathrm{TM}} & f_{v, q^{\prime}, \mathrm{TM}}
\end{array}\right] \hat{O}_{e h}\left[\begin{array}{c}
g_{u, q, \mathrm{TM}} \\
g_{v, q, \mathrm{TM}}
\end{array}\right] d \mathbf{r}_{\perp}=\left\langle\mathbf{f}_{q^{\prime}, \mathrm{TM}}\left|\hat{O}_{e h}\right| \mathbf{g}_{q, \mathrm{TM}}\right\rangle .
$$

Similarly, we can write Eq. (77) as

$$
\hat{O}_{h e}\left[\begin{array}{ll}
\bar{f}_{u, \mathrm{TM}}^{T} & \bar{g}_{u, \mathrm{TE}}^{T} \\
\bar{f}_{v, \mathrm{TM}}^{T} & \bar{g}_{v, \mathrm{TE}}^{T}
\end{array}\right]\left[\begin{array}{l}
-E_{\perp} \\
\bar{c}_{\mathrm{TM}} \\
\bar{c}_{\perp}
\end{array}\right]=\beta\left[\begin{array}{ll}
\bar{g}_{u, \mathrm{TM}}^{T} & \bar{f}_{u, \mathrm{TE}}^{T} \\
\bar{g}_{v, \mathrm{TM}}^{T} & \bar{f}_{v, \mathrm{TE}}^{T}
\end{array}\right]\left[\begin{array}{l}
-H_{\perp} \\
c_{\mathrm{TM}} \\
-H_{\perp} \\
c_{\mathrm{TE}}
\end{array}\right]
$$

leading, using similar steps, to

and

$$
\overline{\bar{O}}_{h e}=\left[\begin{array}{ll}
\overline{\bar{O}}_{h e}^{\mathrm{TMTM}} & \overline{\bar{O}}_{h e}^{\mathrm{TMTE}} \\
\overline{\bar{O}}_{h e}^{\mathrm{TETM}} & \overline{\bar{O}}_{h e}^{\mathrm{TETE}}
\end{array}\right]=\int\left[\begin{array}{ll}
\bar{g}_{u, \mathrm{TM}} & \bar{g}_{v, \mathrm{TM}} \\
\bar{f}_{u, \mathrm{TE}} & \bar{f}_{v, \mathrm{TE}}
\end{array}\right] \hat{O}_{h e}\left[\begin{array}{ll}
\bar{f}_{u, \mathrm{TM}}^{T} & \bar{g}_{u, \mathrm{TE}}^{T} \\
\bar{f}_{v, \mathrm{TM}}^{T} & \bar{g}_{v, \mathrm{TE}}^{T}
\end{array}\right] d \mathbf{r}_{\perp},
$$

$$
\overline{\bar{O}}_{h e}\left[\begin{array}{c}
-E_{\perp} \\
c_{\mathrm{TM}} \\
-E_{\perp} \\
c_{\mathrm{TE}}
\end{array}\right]=\beta\left[\begin{array}{l}
-H_{\perp} \\
c_{\mathrm{TM}} \\
-H_{\perp} \\
c_{\mathrm{TE}}
\end{array}\right]
$$

Once we have computed the operator matrices $\overline{\overline{O_{e h}}}$ and $\overline{\overline{O_{h e}}}$, by combining Eqs. (86) and (90), we can compute the electrical field expansion eigenvectors by solving the eigenvalue problem 


$$
\overline{\bar{O}}_{e h} \overline{\bar{O}}_{h e}\left[\begin{array}{l}
-E_{\perp} \\
\bar{c}_{\mathrm{TM}} \\
-E_{\perp} \\
c_{\mathrm{TE}}
\end{array}\right]=\beta^{2}\left[\begin{array}{l}
-E_{\perp} \\
\bar{c}_{\mathrm{TM}} \\
-E_{\perp} \\
c_{\mathrm{TE}}
\end{array}\right]
$$

using the MATLAB “eig" command. Subsequently, the magnetic field expansion eigenvectors can be obtained from Eq. (90).

\subsection{Evaluation of $O_{e h}$ and $O_{\text {he }}$ matrix elements}

In the following we compute the operator matrix elements of the various matrices $\overline{\bar{O}}_{e h}^{\text {TXTY }}$ and $\overline{\bar{O}}_{h e}^{\text {TXTY }}$. We assume that the dielectric constant $\varepsilon_{r}(u)$ depends only on $u$, which is the case for rotationally symmetric and elliptical geometries. Integration by parts Eqs. (16)-(18) is used extensively in the derivations, where we always assume that the boundary terms vanish as discussed in Section 1.2.

The TMTM contribution for the $\overline{\bar{O}}_{e h}$ operator is given by

$$
\begin{aligned}
& O_{e h, q^{\prime} q}^{\mathrm{TMTM}}=\left\langle\mathbf{f}_{q^{\prime}, \mathrm{TM}}\left|\hat{O}_{e h}\right| \mathbf{g}_{q, \mathrm{TM}}\right\rangle=\frac{1}{k_{\perp, q^{\prime}, \mathrm{TM}} k_{\perp, q, \mathrm{TM}}} \int_{l_{u} l_{v}}\left(\left[\frac{1}{l_{u}} \frac{\partial}{\partial u} \frac{1}{l_{v}} \frac{\partial}{\partial v}\right] U_{q^{\prime}, \mathrm{TM}} V_{q^{\prime}, \mathrm{TM}}\right) \\
& {\left[\begin{array}{cc}
-\frac{1}{l_{u}} \frac{\partial}{\partial u} \frac{1}{k_{0}^{2} \varepsilon_{r}} \frac{1}{l_{u} l_{v}} \frac{\partial}{\partial v} l_{u} & 1+\frac{1}{l_{u}} \frac{\partial}{\partial u} \frac{1}{k_{0}^{2} \varepsilon_{r}} \frac{1}{l_{u} l_{v}} \frac{\partial}{\partial u} l_{v} \\
-1-\frac{1}{l_{v}} \frac{\partial}{\partial v} \frac{1}{k_{0}^{2} \varepsilon_{r}} \frac{1}{l_{u} l_{v}} \frac{\partial}{\partial v} l_{u} & \frac{1}{l_{v}} \frac{\partial}{\partial v} \frac{1}{k_{0}^{2} \varepsilon_{r}} \frac{1}{l_{u} l_{v}} \frac{\partial}{\partial u} l_{v}
\end{array}\right]\left[\begin{array}{c}
-\frac{1}{l_{v}} \frac{\partial}{\partial v} \\
\frac{1}{l_{u}} \frac{\partial}{\partial u}
\end{array}\right] U_{q, \mathrm{TM} V_{q, \mathrm{TM}} d u d v}}
\end{aligned}
$$

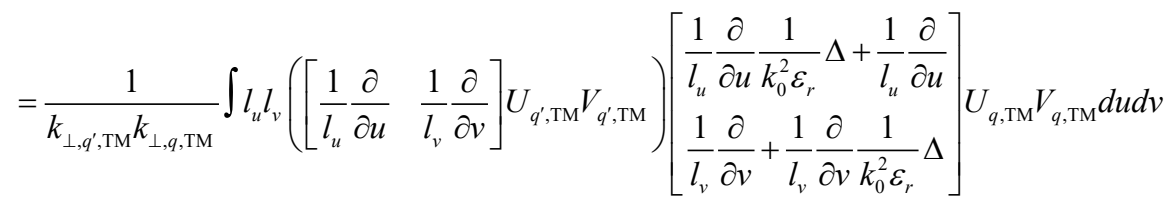

$$
\begin{aligned}
& =\frac{1}{k_{\perp, q^{\prime}, \mathrm{TM}} k_{\perp, q, \mathrm{TM}}} \int\left(\begin{array}{l}
\frac{l_{v}}{l_{u}}\left(\frac{\partial}{\partial u} U_{q^{\prime}, \mathrm{TM}} V_{q^{\prime}, \mathrm{TM}}\right)\left(\frac{\partial}{\partial u}+\frac{\partial}{\partial u} \frac{1}{k_{0}^{2} \varepsilon_{r}} \Delta\right) U_{q, \mathrm{TM}} V_{q, \mathrm{TM}} \\
+\frac{l_{u}}{l_{v}}\left(\frac{\partial}{\partial v} U_{q^{\prime}, \mathrm{TM}} V_{q^{\prime}, \mathrm{TM}}\right)\left(\frac{\partial}{\partial v}+\frac{\partial}{\partial v} \frac{1}{k_{0}^{2} \varepsilon_{r}} \Delta\right) U_{q, \mathrm{TM}} V_{q, \mathrm{TM}}
\end{array}\right) d u d v \\
& =-\frac{1}{k_{\perp, q^{\prime}, \mathrm{TM}} k_{\perp, q, \mathrm{TM}}} \int\left(\left[\frac{\partial}{\partial u} \frac{l_{v}}{l} \frac{\partial}{\partial u}+\frac{\partial}{\partial v} \frac{l_{u}}{l_{v}} \frac{\partial}{\partial v}\right] U_{q^{\prime}, \mathrm{TM}} V_{q^{\prime}, \mathrm{TM}}\right)\left(1+\frac{1}{k_{0}^{2} \varepsilon_{r}} \Delta\right) U_{q, \mathrm{TM}} V_{q, \mathrm{TM}} d u d v \\
& =-\frac{1}{k_{\perp, q^{\prime}, \mathrm{TM}} k_{\perp, q, \mathrm{TM}}} \int l_{u} l_{v}\left(\Delta U_{q^{\prime}, \mathrm{TM}} V_{q^{\prime}, \mathrm{TM}}\right)\left(1+\frac{1}{k_{0}^{2} \varepsilon_{r}} \Delta\right) U_{q, \mathrm{TM}} V_{q, \mathrm{TM}} d u d v \\
& =-k_{\perp, q^{\prime}, \mathrm{TM}} k_{\perp, q, \mathrm{TM}} \int l_{u} l_{v} U_{q^{\prime}, \mathrm{TM}} V_{q^{\prime}, \mathrm{TM}} \frac{1}{k_{0}^{2} \varepsilon_{r}} U_{q, \mathrm{TM}} V_{q, \mathrm{TM}} d u d v+\delta_{q^{\prime} q}
\end{aligned}
$$

The TE-TE contribution is

$$
\begin{aligned}
& O_{e h, q^{\prime} q}^{\mathrm{TETE}}=\left\langle\mathbf{g}_{q^{\prime}, \mathrm{TE}}\left|\hat{O}_{e h}\right| \mathbf{f}_{q, \mathrm{TE}}\right\rangle=\frac{1}{k_{\perp, q^{\prime}, \mathrm{TE}} k_{\perp, q, \mathrm{TE}}} \int_{l_{u}} l_{v}\left(\left[\frac{1}{l_{v}} \frac{\partial}{\partial v}-\frac{1}{l_{u}} \frac{\partial}{\partial u}\right] U_{q^{\prime}, \mathrm{TE}}(u) V_{q^{\prime}, \mathrm{TE}}(v)\right) \\
& {\left[\begin{array}{cc}
-\frac{1}{l_{u}} \frac{\partial}{\partial u} \frac{1}{k_{0}^{2} \varepsilon_{r}} \frac{1}{l_{u} l_{v}} \frac{\partial}{\partial v} l_{u} & 1+\frac{1}{l_{u}} \frac{\partial}{\partial u} \frac{1}{k_{0}^{2} \varepsilon_{r}} \frac{1}{l_{u} l_{v}} \frac{\partial}{\partial u} l_{v} \\
-1-\frac{1}{l_{v}} \frac{\partial}{\partial v} \frac{1}{k_{0}^{2} \varepsilon_{r}} \frac{1}{l_{u} l_{v}} \frac{\partial}{\partial v} l_{u} & \frac{1}{l_{v}} \frac{\partial}{\partial v} \frac{1}{k_{0}^{2} \varepsilon_{r}} \frac{1}{l_{u} l_{v}} \frac{\partial}{\partial u} l_{v}
\end{array}\right]\left[\begin{array}{c}
\frac{1}{l_{u}} \frac{\partial}{\partial u} \\
\frac{1}{l_{v}} \frac{\partial}{\partial v}
\end{array}\right] U_{q, \mathrm{TE}}(u) V_{q, \mathrm{TE}}(v) d u d v} \\
& =\frac{1}{k_{\perp, q^{\prime}, \mathrm{TE}} k_{\perp, q, \mathrm{TE}}} \int_{l_{u} l_{v}}\left(\left[\frac{1}{l_{v}} \frac{\partial}{\partial v}-\frac{1}{l_{u}} \frac{\partial}{\partial u}\right] U_{q^{\prime}, \mathrm{TE}}(u) V_{q^{\prime}, \mathrm{TE}}(v)\right)\left[\begin{array}{c}
\frac{1}{l_{v}} \frac{\partial}{\partial v} \\
-\frac{1}{l_{u}} \frac{\partial}{\partial u}
\end{array}\right] U_{q, \mathrm{TE}}(u) V_{q, \mathrm{TE}}(v) d u d v
\end{aligned}
$$




$$
=-\frac{1}{k_{\perp, q, T E} k_{\perp, q, T E}} \int l_{u} l_{v} U_{q^{\prime}, T E} V_{q^{\prime}, T E} \Delta U_{q, T E} V_{q, T E} d u d v=\delta_{q^{\prime} q}
$$

The TM-TE contribution is

$$
\begin{aligned}
& O_{e h, q^{\prime} q}^{\mathrm{TMT}}=\left\langle\mathbf{f}_{q^{\prime}, \mathrm{TM}}\left|\hat{O}_{e h}\right| \mathbf{f}_{q, \mathrm{TE}}\right\rangle=\frac{1}{k_{\perp, q^{\prime}, \mathrm{TM}} k_{\perp, q, \mathrm{TE}}} \int_{l_{u} l_{v}}\left(\left[\frac{1}{l_{u}} \frac{\partial}{\partial u} \frac{1}{l_{v}} \frac{\partial}{\partial v}\right] U_{q^{\prime}, \mathrm{TM}}(u) V_{q^{\prime}, \mathrm{TM}}(v)\right) \\
& {\left[\begin{array}{cc}
-\frac{1}{l_{u}} \frac{\partial}{\partial u} \frac{1}{k_{0}^{2} \varepsilon_{r}} \frac{1}{l_{u} l_{v} \frac{\partial}{\partial v} l_{u}} & 1+\frac{1}{l_{u}} \frac{\partial}{\partial u} \frac{1}{k_{0}^{2} \varepsilon_{r}} \frac{1}{l_{u} l_{v}} \frac{\partial}{\partial u} l_{v} \\
-1-\frac{1}{l_{v}} \frac{\partial}{\partial v} \frac{1}{k_{0}^{2} \varepsilon_{r}} \frac{1}{l_{u} l_{v}} \frac{\partial}{\partial v} l_{u} & \frac{1}{l_{v}} \frac{\partial}{\partial v} \frac{1}{k_{0}^{2} \varepsilon_{r}} \frac{1}{l_{u} l_{v}} \frac{\partial}{\partial u} l_{v}
\end{array}\right]\left[\begin{array}{c}
\frac{1}{l_{u}} \frac{\partial}{\partial u} \\
\frac{1}{l_{v}} \frac{\partial}{\partial v}
\end{array}\right] U_{q, \mathrm{TE}}(u) V_{q, \mathrm{TE}}(v) d u d v} \\
& =\frac{1}{k_{\perp, q^{\prime}, \mathrm{TM}} k_{\perp, q, \mathrm{TE}}} \int_{l_{u} l_{v}}\left(\left[\frac{1}{l_{u}} \frac{\partial}{\partial u} \frac{1}{l_{v}} \frac{\partial}{\partial v}\right] U_{q^{\prime}, \mathrm{TM}}(u) V_{q^{\prime}, \mathrm{TM}}(v)\right)\left[\begin{array}{c}
\frac{1}{l_{v}} \frac{\partial}{\partial v} \\
-\frac{1}{l_{u}} \frac{\partial}{\partial u}
\end{array}\right] U_{q, \mathrm{TE}}(u) V_{q, \mathrm{TE}}(v) d u d v=0
\end{aligned}
$$

The TE-TM contribution is

$$
\begin{aligned}
& O_{e h, q^{\prime} q}^{\mathrm{TETM}}=\left\langle\mathbf{g}_{q^{\prime}, \mathrm{TE}}\left|\hat{O}_{e h}\right| \mathbf{g}_{q, \mathrm{TM}}\right\rangle=\frac{1}{k_{\perp, q^{\prime}, \mathrm{TE}} k_{\perp, q, \mathrm{TM}}} \int l_{u} l_{v}\left(\left[\frac{1}{l_{v}} \frac{\partial}{\partial v}-\frac{1}{l_{u}} \frac{\partial}{\partial u}\right] U_{q^{\prime}, \mathrm{TE}}(u) V_{q^{\prime}, \mathrm{TE}}(v)\right) \\
& {\left[\begin{array}{cc}
-\frac{1}{l_{u}} \frac{\partial}{\partial u} \frac{1}{k_{0}^{2} \varepsilon_{r}} \frac{1}{l_{u} l_{v}} \frac{\partial}{\partial v} l_{u} & 1+\frac{1}{l_{u}} \frac{\partial}{\partial u} \frac{1}{k_{0}^{2} \varepsilon_{r}} \frac{1}{l_{u} l_{v}} \frac{\partial}{\partial u} l_{v} \\
-1-\frac{1}{l_{v}} \frac{\partial}{\partial v} \frac{1}{k_{0}^{2} \varepsilon_{r}} \frac{1}{l_{u} l_{v}} \frac{\partial}{\partial v} l_{u} & \frac{1}{l_{v}} \frac{\partial}{\partial v} \frac{1}{k_{0}^{2} \varepsilon_{r}} \frac{1}{l_{u} l_{v}} \frac{\partial}{\partial u} l_{v}
\end{array}\right]\left[\begin{array}{c}
-\frac{1}{l_{v}} \frac{\partial}{\partial v} \\
\frac{1}{l_{u}} \frac{\partial}{\partial u}
\end{array}\right] U_{q, \mathrm{TM}}(u) V_{q, \mathrm{TM}}(v) d u d v} \\
& =\frac{1}{k_{\perp, q, \mathrm{TE}} k_{\perp, q, \mathrm{TM}}} \int l_{u} l_{v}\left(\left[\frac{1}{l_{v}} \frac{\partial}{\partial v}-\frac{1}{l_{u}} \frac{\partial}{\partial u}\right] U_{q^{\prime}, \mathrm{TE}}(u) V_{q, \mathrm{TE}}(v)\right)\left[\begin{array}{l}
\frac{1}{l_{u}} \frac{\partial}{\partial u} \frac{1}{k_{0}^{2} \varepsilon_{r}} \Delta+\frac{1}{l_{u}} \frac{\partial}{\partial u} \\
\frac{1}{l_{v}} \frac{\partial}{\partial v}+\frac{1}{l_{v}} \frac{\partial}{\partial v} \frac{1}{k_{0}^{2} \varepsilon_{r}} \Delta
\end{array}\right] U_{q, \mathrm{TM}}(u) V_{q, \mathrm{TM}}(v) d u d v \\
& =-\frac{1}{k_{\perp, q^{\prime}, \mathrm{TE}} k_{\perp, q, \mathrm{TM}}} \int\left[\left(\frac{\partial}{\partial u} \frac{\partial}{\partial v} U_{q^{\prime}, \mathrm{TE}} V_{q^{\prime}, \mathrm{TE}}\right)-\left(\frac{\partial}{\partial v} \frac{\partial}{\partial u} U_{q^{\prime}, \mathrm{TE}} V_{q^{\prime}, \mathrm{TE}}\right)\right]\left(1+\frac{1}{k_{0}^{2} \varepsilon_{r}} \Delta\right) U_{q, \mathrm{TM}} V_{q, \mathrm{TM}} d u d v=0
\end{aligned}
$$

The TM-TM contribution for the $\hat{O}_{h e}$ operator is given by

$$
\begin{aligned}
& O_{h e, q^{\prime} q}^{\mathrm{TMTM}}=\left\langle\mathbf{g}_{q^{\prime}, \mathrm{TM}}\left|\hat{O}_{h e}\right| \mathbf{f}_{q, \mathrm{TM}}\right\rangle=\frac{1}{k_{\perp, q^{\prime}, \mathrm{TM}} k_{\perp, q, \mathrm{TM}}} \int_{l_{u} l_{v}}\left(\left[-\frac{1}{l_{v}} \frac{\partial}{\partial v} \frac{1}{l_{u}} \frac{\partial}{\partial u}\right] U_{q^{\prime}, \mathrm{TM}}(u) V_{q^{\prime}, \mathrm{TM}}(v)\right) \\
& {\left[\begin{array}{cc}
\frac{1}{l_{u}} \frac{\partial}{\partial u} \frac{1}{l_{u} l_{v}} \frac{\partial}{\partial v} l_{u} & -k_{0}^{2} \varepsilon_{r}-\frac{1}{l_{u}} \frac{\partial}{\partial u} \frac{1}{l_{u} l_{v}} \frac{\partial}{\partial u} l_{v} \\
k_{0}^{2} \varepsilon_{r}+\frac{1}{l_{v}} \frac{\partial}{\partial v} \frac{1}{l_{u} l_{v}} \frac{\partial}{\partial v} l_{u} & -\frac{1}{l_{v}} \frac{\partial}{\partial v} \frac{1}{l_{u} l_{v}} \frac{\partial}{\partial u} l_{v}
\end{array}\right]\left[\begin{array}{c}
\frac{1}{l_{u}} \frac{\partial}{\partial u} \\
\frac{1}{l_{v}} \frac{\partial}{\partial v}
\end{array}\right] U_{q, \mathrm{TM}}(u) V_{q, \mathrm{TM}}(v) d u d v} \\
& =\frac{1}{k_{\perp, q^{\prime}, \mathrm{TM}} k_{\perp, q, \mathrm{TM}}} \int_{l_{u} l_{v}}\left(\left[\begin{array}{lll}
-\frac{1}{l_{v}} \frac{\partial}{\partial v} & \frac{1}{l_{l}} \frac{\partial}{\partial u}
\end{array}\right] U_{q^{\prime}, \mathrm{TM}}(u) V_{q^{\prime}, \mathrm{TM}}(v)\right)\left[\begin{array}{l}
-k_{0}^{2} \varepsilon_{r} \frac{1}{l_{v}} \frac{\partial}{\partial v} \\
k_{0}^{2} \varepsilon_{r} \frac{1}{l_{u}} \frac{\partial}{\partial u}
\end{array}\right] U_{q, \mathrm{TM}}(u) V_{q, \mathrm{TM}}(v) d u d v \\
& =\frac{1}{k_{\perp, q^{\prime}, \mathrm{TM}} k_{\perp, q, \mathrm{TM}}} \int\left[\left(\frac{\partial}{\partial v} U_{q^{\prime}, \mathrm{TM}} V_{q^{\prime}, \mathrm{TM}}\right) k_{0}^{2} \varepsilon_{r} \frac{l_{u}}{l_{v}}\left(\frac{\partial}{\partial v} U_{q, \mathrm{TM}} V_{q, \mathrm{TM}}\right)+\left(\frac{\partial}{\partial u} U_{q^{\prime}, \mathrm{TM}} V_{q^{\prime}, \mathrm{TM}}\right) k_{0}^{2} \varepsilon_{r} \frac{l_{v}}{l_{u}}\left(\frac{\partial}{\partial u} U_{q, \mathrm{TM}} V_{q, \mathrm{TM}}\right)\right] d u d v \\
& =-\frac{1}{k_{\perp, q^{\prime}, \mathrm{TM}} k_{\perp, q, \mathrm{TM}}} \int l_{u} l_{v} U_{q^{\prime}, \mathrm{TM}} V_{q^{\prime}, \mathrm{TM}} k_{0}^{2} \varepsilon_{r} \Delta U_{q, \mathrm{TM}} V_{q, \mathrm{TM}} d u d v-\frac{1}{k_{\perp, q^{\prime}, \mathrm{TM}} k_{\perp, q, \mathrm{TM}}} \int U_{q^{\prime}, \mathrm{TM}} V_{q^{\prime}, \mathrm{TM}} k_{0}^{2}\left(\frac{\partial}{\partial u} \varepsilon_{r}\right) \frac{l_{v}}{l_{u}}\left(\frac{\partial}{\partial u} U_{q, \mathrm{TM}}\right) V_{q, \mathrm{TM}} d u d v \\
& =\frac{k_{\perp, q, \mathrm{TM}}}{k_{\perp, q^{\prime}, \mathrm{TM}}} \int l_{u} l_{v} U_{q^{\prime}, \mathrm{TM}} V_{q^{\prime}, \mathrm{TM}} k_{0}^{2} \varepsilon_{r} U_{q, \mathrm{TM}} V_{q, \mathrm{TM}} d u d v-\frac{1}{k_{\perp, q^{\prime}, \mathrm{TM}} k_{\perp, q, \mathrm{TM}}} \int U_{q^{\prime}, \mathrm{TM}} V_{q^{\prime}, \mathrm{TM}} k_{0}^{2}\left(\frac{\partial}{\partial u} \varepsilon_{r}\right) \frac{l_{v}}{l_{u}}\left(\frac{\partial}{\partial u} U_{q, \mathrm{TM}}\right) V_{q, \mathrm{TM}} d u d v
\end{aligned}
$$

The TE-TE contribution is 


$$
\begin{aligned}
& O_{h e, q^{\prime} q}^{\mathrm{TETE}}=\left\langle\mathbf{f}_{q^{\prime}, \mathrm{TE}}\left|\hat{O}_{h e}\right| \mathbf{g}_{q, \mathrm{TE}}\right\rangle=\frac{1}{k_{\perp, q^{\prime}, \mathrm{TE}} k_{\perp, q, \mathrm{TE}}} \int l_{u} l_{v}\left[\frac{1}{l_{u}} \frac{\partial}{\partial u} \frac{1}{l_{v}} \frac{\partial}{\partial v}\right] U_{q^{\prime}, \mathrm{TE}}(u) V_{q^{\prime}, \mathrm{TE}}(v) \\
& {\left[\begin{array}{cc}
\frac{1}{l_{u}} \frac{\partial}{\partial u} \frac{1}{l_{u} l_{v}} \frac{\partial}{\partial v} l_{u} & -k_{0}^{2} \varepsilon_{r}-\frac{1}{l_{u}} \frac{\partial}{\partial u} \frac{1}{l_{u} l_{v}} \frac{\partial}{\partial u} l_{v} \\
k_{0}^{2} \varepsilon_{r}+\frac{1}{l_{v}} \frac{\partial}{\partial v} \frac{1}{l_{u} l_{v}} \frac{\partial}{\partial v} l_{u} & -\frac{1}{l_{v}} \frac{\partial}{\partial v} \frac{1}{l_{u} l_{v}} \frac{\partial}{\partial u} l_{v}
\end{array}\right]\left[\begin{array}{c}
\frac{1}{l_{v}} \frac{\partial}{\partial v} \\
-\frac{1}{l_{u}} \frac{\partial}{\partial u}
\end{array}\right] U_{q, \mathrm{TE}}(u) V_{q, \mathrm{TE}}(v) d u d v} \\
& =\frac{1}{k_{\perp, q^{\prime}, \mathrm{TE}} k_{\perp, q, \mathrm{TE}}} \int l_{u} l_{v}\left[\frac{1}{l_{u}} \frac{\partial}{\partial u} \frac{1}{l_{v}} \frac{\partial}{\partial v}\right] U_{q^{\prime}, \mathrm{TE}}(u) V_{q^{\prime}, \mathrm{TE}}(v)\left[\begin{array}{l}
\frac{1}{l_{u}} \frac{\partial}{\partial u} \frac{1}{l_{u} l_{v}}\left(\frac{\partial}{\partial v} \frac{l_{u}}{l_{v}} \frac{\partial}{\partial v}+\frac{\partial}{\partial u} \frac{l_{v}}{l_{u}} \frac{\partial}{\partial u}\right)+k_{0}^{2} \varepsilon_{r} \frac{1}{l_{u}} \frac{\partial}{\partial u} \\
\frac{1}{l_{v}} \frac{\partial}{\partial v} \frac{1}{l_{u} l_{v}}\left(\frac{\partial}{\partial v} \frac{l_{u}}{l_{v}} \frac{\partial}{\partial v}+\frac{\partial}{\partial u} \frac{l_{v}}{l_{u}} \frac{\partial}{\partial u}\right)+k_{0}^{2} \varepsilon_{r} \frac{1}{l_{v}} \frac{\partial}{\partial v}
\end{array}\right] U_{q, \mathrm{TE}}(u) V_{q, \mathrm{TE}}(v) d u d v \\
& =\frac{1}{k_{\perp, q^{\prime}, \mathrm{TE}} k_{\perp, q, \mathrm{TE}}} \int\left(\begin{array}{l}
\left(\frac{\partial}{\partial u} U_{q^{\prime}, \mathrm{TE}} V_{q^{\prime}, \mathrm{TE}}\right) \frac{l_{v}}{l_{u}}\left(k_{0}^{2} \varepsilon_{r} \frac{\partial}{\partial u} U_{q, \mathrm{TE}} V_{q, \mathrm{TE}}+\frac{\partial}{\partial u} \Delta U_{q, \mathrm{TE}} V_{q, \mathrm{TE}}\right) \\
+\left(\frac{\partial}{\partial v} U_{q^{\prime}, \mathrm{TE}} V_{q^{\prime}, \mathrm{TE}}\right) \frac{l_{u}}{l_{v}}\left(k_{0}^{2} \varepsilon_{r} \frac{\partial}{\partial v} U_{q, \mathrm{TE}} V_{q, \mathrm{TE}}+\frac{\partial}{\partial v} \Delta U_{q, \mathrm{TE}} V_{q, \mathrm{TE}}\right)
\end{array}\right) d u d v \\
& =-\frac{1}{k_{\perp, q^{\prime}, \mathrm{TE}} k_{\perp, q, \mathrm{TE}}} \int U_{q^{\prime}, \mathrm{TE}} V_{q^{\prime}, \mathrm{TE}}\left(\frac{\partial}{\partial u} \frac{l_{v}}{l_{u}} \frac{\partial}{\partial u}+\frac{\partial}{\partial v} \frac{l_{u}}{l_{v}} \frac{\partial}{\partial v}\right) \Delta U_{q, \mathrm{TE}} V_{q, \mathrm{TE}} d u d v \\
& -\frac{1}{k_{\perp, q^{\prime}, \mathrm{TE}} k_{\perp, q, \mathrm{TE}}} \int l_{u} l_{v} U_{q^{\prime}, \mathrm{TE}} V_{q^{\prime}, \mathrm{TE}} k_{0}^{2} \varepsilon_{r} \Delta U_{q, \mathrm{TE}} V_{q, \mathrm{TE}} d u d v-\frac{1}{k_{\perp, q^{\prime}, \mathrm{TE}} k_{\perp, q, \mathrm{TE}}} \int U_{q^{\prime}, \mathrm{TE}} V_{q^{\prime}, \mathrm{TE}} k_{0}^{2}\left(\frac{\partial}{\partial u} \varepsilon_{r}\right) \frac{l_{v}}{l_{u}}\left(\frac{\partial}{\partial u} U_{q, \mathrm{TE}}\right) V_{q, \mathrm{TE}} d u d v \\
& =-\frac{1}{k_{\perp, q^{\prime}, \mathrm{TE}} k_{\perp, q, \mathrm{TE}}} \int l_{u} l_{v} U_{q^{\prime}, \mathrm{TE}} V_{q^{\prime}, \mathrm{TE}} \Delta \Delta U_{q, \mathrm{TE}} V_{q, \mathrm{TE}} d u d v-\frac{1}{k_{\perp, q^{\prime}, \mathrm{TE}} k_{\perp, q, \mathrm{TE}}} \int l_{u} l_{v} U_{q^{\prime}, \mathrm{TE}} V_{q^{\prime}, \mathrm{TE}} k_{0}^{2} \varepsilon_{r} \Delta U_{q, \mathrm{TE}} V_{q, \mathrm{TE}} d u d v \\
& -\frac{1}{k_{\perp, q^{\prime}, \mathrm{TE}} k_{\perp, q, \mathrm{TE}}} \int U_{q^{\prime}, \mathrm{TE}} V_{q^{\prime}, \mathrm{TE}} k_{0}^{2}\left(\frac{\partial}{\partial u} \varepsilon_{r}\right) \frac{l_{v}}{l_{u}}\left(\frac{\partial}{\partial u} U_{q, \mathrm{TE}}\right) V_{q, \mathrm{TE}} d u d v \\
& =-k_{\perp, q, \mathrm{TE}}^{2} \delta_{q q^{\prime}}+\frac{k_{\perp, q, \mathrm{TE}}}{k_{\perp, q^{\prime}, \mathrm{TE}}} \int l_{u} l_{v} U_{q^{\prime}, \mathrm{TE}} V_{q^{\prime}, \mathrm{TE}} k_{0}^{2} \varepsilon_{r} U_{q, \mathrm{TE}} V_{q, \mathrm{TE}} d u d v \\
& -\frac{1}{k_{\perp, q^{\prime}, \mathrm{TE}} k_{\perp, q, \mathrm{TE}}} \int U_{q^{\prime}, \mathrm{TE}} V_{q^{\prime}, \mathrm{TE}} k_{0}^{2}\left(\frac{\partial}{\partial u} \varepsilon_{r}\right) \frac{l_{v}}{l_{u}}\left(\frac{\partial}{\partial u} U_{q, \mathrm{TE}}\right) V_{q, \mathrm{TE}} d u d v
\end{aligned}
$$

\section{The TM-TE contribution is}

$$
\begin{aligned}
& O_{h e, q^{\prime} q}^{\mathrm{TMTE}}=\left\langle\mathbf{g}_{q^{\prime}, \mathrm{TM}}\left|\hat{O}_{h e}\right| \mathbf{g}_{q, \mathrm{TE}}\right\rangle=\frac{1}{k_{\perp, q^{\prime}, \mathrm{TM}} k_{\perp, q, \mathrm{TE}}} \int_{l_{u} l_{v}}\left(\left[-\frac{1}{l_{v}} \frac{\partial}{\partial v} \frac{1}{l_{u}} \frac{\partial}{\partial u}\right] U_{q^{\prime}, \mathrm{TM}}(u) V_{q^{\prime}, \mathrm{TM}}(v)\right) \\
& {\left[\begin{array}{cc}
\frac{1}{l_{u}} \frac{\partial}{\partial u} \frac{1}{l_{u} l_{v}} \frac{\partial}{\partial v} l_{u} & -k_{0}^{2} \varepsilon_{r}-\frac{1}{l_{u}} \frac{\partial}{\partial u} \frac{1}{l_{u} l_{v}} \frac{\partial}{\partial u} l_{v} \\
k_{0}^{2} \varepsilon_{r}+\frac{1}{l_{v}} \frac{\partial}{\partial v} \frac{1}{l_{u} l_{v}} \frac{\partial}{\partial v} l_{u} & -\frac{1}{l_{v}} \frac{\partial}{\partial v} \frac{1}{l_{u} l_{v}} \frac{\partial}{\partial u} l_{v}
\end{array}\right]\left[\begin{array}{c}
\frac{1}{l_{v}} \frac{\partial}{\partial v} \\
-\frac{1}{l_{u}} \frac{\partial}{\partial u}
\end{array}\right] U_{q, \mathrm{TE}}(u) V_{q, \mathrm{TE}}(v) d u d v} \\
& =\frac{1}{k_{\perp, q^{\prime}, \mathrm{TM}} k_{\perp, q, \mathrm{TE}}} \int_{l_{u} l_{v}}\left(\left[-\frac{1}{l_{v}} \frac{\partial}{\partial v} \frac{1}{l_{u}} \frac{\partial}{\partial u}\right] U_{q^{\prime}, \mathrm{TM}}(u) V_{q^{\prime}, \mathrm{TM}}(v)\right)\left[\begin{array}{l}
\frac{1}{l_{u}} \frac{\partial}{\partial u} \Delta+k_{0}^{2} \varepsilon_{r} \frac{1}{l_{u}} \frac{\partial}{\partial u} \\
\frac{1}{l_{v}} \frac{\partial}{\partial v} \Delta+k_{0}^{2} \varepsilon_{r} \frac{1}{l_{v}} \frac{\partial}{\partial v}
\end{array}\right] U_{q, \mathrm{TE}}(u) V_{q, \mathrm{TE}}(v) d u d v \\
& =\frac{1}{k_{\perp, q^{\prime}, \mathrm{TM}} k_{\perp, q, \mathrm{TE}}} \int\left[\left(\frac{\partial}{\partial u} \frac{\partial}{\partial v} U_{q^{\prime}, \mathrm{TM}} V_{q^{\prime}, \mathrm{TM}}\right)-\left(\frac{\partial}{\partial v} \frac{\partial}{\partial u} U_{q^{\prime}, \mathrm{TM}} V_{q^{\prime}, \mathrm{TM}}\right)\right] \Delta U_{q, \mathrm{TE}} V_{q, \mathrm{TE}} d u d v \\
& -\frac{1}{k_{\perp, q^{\prime}, \mathrm{TM}} k_{\perp, q, \mathrm{TE}}} \int\left[\left(\frac{\partial}{\partial v} U_{q^{\prime}, \mathrm{TM}} V_{q^{\prime}, \mathrm{TM}}\right) k_{0}^{2} \varepsilon_{r}\left(\frac{\partial}{\partial u} U_{q, \mathrm{TE}} V_{q, \mathrm{TE}}\right)-\left(\frac{\partial}{\partial u} U_{q^{\prime}, \mathrm{TM}} V_{q^{\prime}, \mathrm{TM}}\right) k_{0}^{2} \varepsilon_{r}\left(\frac{\partial}{\partial v} U_{q, \mathrm{TE}} V_{q, \mathrm{TE}}\right)\right] d u d v \\
& =-\frac{1}{k_{\perp, q^{\prime}, \mathrm{TM}} k_{\perp, q, \mathrm{TE}}} \int U_{q^{\prime}, \mathrm{TM}} V_{q^{\prime}, \mathrm{TM}} k_{0}^{2}\left(\frac{\partial}{\partial u} \varepsilon_{r}\right) U_{q, \mathrm{TE}}\left(\frac{\partial}{\partial v} V_{q, \mathrm{TE}}\right) d u d v
\end{aligned}
$$

The TE-TM contribution is 


$$
\begin{aligned}
& O_{h e, q^{\prime} q}^{\mathrm{TETM}}=\left\langle\mathbf{f}_{q^{\prime}, \mathrm{TE}}\left|\hat{O}_{h e}\right| \mathbf{f}_{q, \mathrm{TM}}\right\rangle=\frac{1}{k_{\perp, q^{\prime}, \mathrm{TE}} k_{\perp, q, \mathrm{TM}}} \int\left(\left[\frac{1}{l_{u}} \frac{\partial}{\partial u} \frac{1}{l_{v}} \frac{\partial}{\partial v}\right] U_{q^{\prime}, \mathrm{TE}}(u) V_{q^{\prime}, \mathrm{TE}}(v)\right) \\
& {\left[\begin{array}{cc}
\frac{1}{l_{u}} \frac{\partial}{\partial u} \frac{1}{l_{u} l_{v}} \frac{\partial}{\partial v} l_{u} & -k_{0}^{2} \varepsilon_{r}-\frac{1}{l_{u}} \frac{\partial}{\partial u} \frac{1}{l_{u} l_{v}} \frac{\partial}{\partial u} l_{v} \\
k_{0}^{2} \varepsilon_{r}+\frac{1}{l_{v}} \frac{\partial}{\partial v} \frac{1}{l_{u} l_{v}} \frac{\partial}{\partial v} l_{u} & -\frac{1}{l_{v}} \frac{\partial}{\partial v} \frac{1}{l_{u} l_{v}} \frac{\partial}{\partial u} l_{v}
\end{array}\right]\left[\begin{array}{c}
\frac{1}{l_{u}} \frac{\partial}{\partial u} \\
\frac{1}{l_{v}} \frac{\partial}{\partial v}
\end{array}\right] U_{q, \mathrm{TM}}(u) V_{q, \mathrm{TM}}(v) l_{u} l_{v} d u d v} \\
& =\frac{1}{k_{\perp, q^{\prime}, \mathrm{TE}} k_{\perp, q, \mathrm{TM}}} \int\left(\left[\frac{1}{l_{u}} \frac{\partial}{\partial u} \frac{1}{l_{v}} \frac{\partial}{\partial v}\right] U_{q^{\prime}, \mathrm{TE}}(u) V_{q^{\prime}, \mathrm{TE}}(v)\right)\left[\begin{array}{c}
-k_{0}^{2} \varepsilon_{r} \frac{1}{l_{v}} \frac{\partial}{\partial v} \\
k_{0}^{2} \varepsilon_{r} \frac{1}{l_{u}} \frac{\partial}{\partial u}
\end{array}\right] U_{q, \mathrm{TM}}(u) V_{q, \mathrm{TM}}(v) l_{u} l_{v} d u d v \\
& =\frac{1}{k_{\perp, q^{\prime}, \mathrm{TE}} k_{\perp, q, \mathrm{TM}}} \int\left[-\left(\frac{\partial}{\partial u} U_{q^{\prime}, \mathrm{TE}} V_{q^{\prime}, \mathrm{TE}}\right) k_{0}^{2} \varepsilon_{r}\left(\frac{\partial}{\partial v} U_{q, \mathrm{TM}} V_{q, \mathrm{TM}}\right)+\left(\frac{\partial}{\partial v} U_{q^{\prime}, \mathrm{TE}} V_{q^{\prime}, \mathrm{TE}}\right) k_{0}^{2} \varepsilon_{r}\left(\frac{\partial}{\partial u} U_{q, \mathrm{TM}} V_{q, \mathrm{TM}}\right)\right] d u d v \\
& =\frac{1}{k_{\perp, q^{\prime}, \mathrm{TE}} k_{\perp, q, \mathrm{TM}}} \int U_{q^{\prime}, \mathrm{TE}} V_{q^{\prime}, \mathrm{TE}} k_{0}^{2}\left(\frac{\partial}{\partial u} \varepsilon_{r}\right) U_{q, \mathrm{TM}}\left(\frac{\partial}{\partial v} V_{q, \mathrm{TM}}\right) d u d v
\end{aligned}
$$

\subsection{Calculation of displacement field}

The planar components of the displacement field can be computed using the planar version of (25) using the scaling (40), which is

$$
\mathbf{D}_{\perp}=\varepsilon_{r}(u) \mathbf{E}_{\perp}=\widehat{O}_{d e} \mathbf{E}_{\perp} .
$$

Using (80) and the expansion for the displacement field given by

$$
\left[\begin{array}{l}
d_{u} \\
d_{v}
\end{array}\right]=\sum_{q} c_{\mathrm{TM}, q}^{D_{\perp}} \mathbf{f}_{q, \mathrm{TM}}(u, v)+\sum_{q} c_{\mathrm{TE}, q}^{D_{\perp}} \mathbf{g}_{q, \mathrm{TE}}(u, v)=\left[\begin{array}{ll}
\bar{f}_{u, \mathrm{TM}}^{T} & \bar{g}_{u, \mathrm{TE}}^{T} \\
\bar{f}_{v, \mathrm{TM}}^{T} & \bar{g}_{v, \mathrm{TE}}^{T}
\end{array}\right]\left[\begin{array}{l}
-D_{\perp} \\
c_{\mathrm{TM}} \\
-D_{\perp} \\
\bar{c}_{\mathrm{TE}}
\end{array}\right],
$$

we can write (100) as

$$
\left[\begin{array}{ll}
\bar{f}_{u, \mathrm{TM}}^{T} & \bar{g}_{u, \mathrm{TE}}^{T} \\
\bar{f}_{v, \mathrm{TM}}^{T} & \bar{g}_{v, \mathrm{TE}}^{T}
\end{array}\right]\left[\begin{array}{l}
\bar{c}_{\perp} \\
\bar{c}_{\mathrm{TM}} \\
\bar{c}_{\perp}
\end{array}\right]=\left[\begin{array}{cc}
\varepsilon_{r} & 0 \\
0 & \varepsilon_{r}
\end{array}\right]\left[\begin{array}{ll}
\bar{f}_{u, \mathrm{TM}}^{T} & \bar{g}_{u, \mathrm{TE}}^{T} \\
\bar{f}_{v, \mathrm{TM}}^{T} & \bar{g}_{v, \mathrm{TE}}^{T}
\end{array}\right]\left[\begin{array}{l}
-E_{\perp} \\
\overline{\mathrm{TM}}_{\mathrm{TM}} \\
-E_{\perp} \\
\bar{c}_{\mathrm{TE}}
\end{array}\right]
$$

As before we multiply (102) with $\int\left[\begin{array}{ll}\bar{f}_{u, \mathrm{TM}} & \bar{f}_{v, \mathrm{TM}} \\ \bar{g}_{u, \mathrm{TE}} & \bar{g}_{v, \mathrm{TE}}\end{array}\right] d \mathbf{r}_{\perp}$ to obtain

$$
\left[\begin{array}{l}
-D_{\perp} \\
\bar{c}_{\mathrm{TM}} \\
\bar{c}_{\mathrm{TE}}
\end{array}\right]=\overline{\bar{O}}_{d e}\left[\begin{array}{l}
\bar{c}_{\perp} \\
\bar{c}_{\mathrm{TM}} \\
-E_{\perp} \\
\bar{c}_{\mathrm{TE}}
\end{array}\right]
$$

where

$$
\overline{\bar{O}}_{d e}=\left[\begin{array}{ll}
\overline{\bar{O}}_{d e}^{\mathrm{TMTM}} & \overline{\bar{O}}_{d e}^{\mathrm{TMTE}} \\
\overline{\bar{O}}_{d e}^{\mathrm{TETM}} & \overline{\bar{O}}_{d e}^{\mathrm{TETE}}
\end{array}\right]=\int\left[\begin{array}{ll}
\bar{f}_{u, \mathrm{TM}} & \bar{f}_{v, \mathrm{TM}} \\
\bar{g}_{u, \mathrm{TE}} & \bar{g}_{v, \mathrm{TE}}
\end{array}\right]\left[\begin{array}{cc}
\varepsilon_{r} & 0 \\
0 & \varepsilon_{r}
\end{array}\right]\left[\begin{array}{ll}
\bar{f}_{u, \mathrm{TM}}^{T} & \bar{g}_{u, \mathrm{TE}}^{T} \\
\bar{f}_{v, \mathrm{TM}}^{T} & \bar{g}_{v, \mathrm{TE}}^{T}
\end{array}\right] d \mathbf{r}_{\perp} .
$$

The TMTM element of the $\overline{\bar{O}}_{d e}$ matrix is given by

$$
O_{d e, q^{\prime} q}^{\mathrm{TMTM}}=\left\langle\mathbf{f}_{q^{\prime}, \mathrm{TM}}\left|\hat{O}_{d e}\right| \mathbf{f}_{q, \mathrm{TM}}\right\rangle=\frac{1}{k_{\perp, q^{\prime}, \mathrm{TM}} k_{\perp, q, \mathrm{TM}}} \int l_{u} l_{v}\left(\left[\begin{array}{ll}
\frac{1}{l_{u}} \frac{\partial}{\partial u} & \frac{1}{l_{v}} \frac{\partial}{\partial v}
\end{array}\right] U_{q^{\prime}, \mathrm{TM}}(u) V_{q^{\prime}, \mathrm{TM}}(v)\right)\left[\begin{array}{cc}
\varepsilon_{r} & 0 \\
0 & \varepsilon_{r}
\end{array}\right]\left[\begin{array}{c}
\frac{1}{l_{u}} \frac{\partial}{\partial u} \\
\frac{1}{l_{v}} \frac{\partial}{\partial v}
\end{array}\right] U_{q, \mathrm{TM}}(u) V_{q, \mathrm{TM}}(v) d u d v
$$




$$
\begin{aligned}
& =\frac{1}{k_{\perp, q^{\prime}, \mathrm{TM}} k_{\perp, q, \mathrm{TM}}} \int\left(\begin{array}{l}
\left(\frac{\partial}{\partial u} U_{q^{\prime}, \mathrm{TM}} V_{q^{\prime}, \mathrm{TM}}\right) \varepsilon_{r} \frac{l_{v}}{l_{u}}\left(\frac{\partial}{\partial u} U_{q, \mathrm{TM}} V_{q, \mathrm{TM}}\right) \\
+\left(\frac{\partial}{\partial v} U_{q^{\prime}, \mathrm{TM}} V_{q^{\prime}, \mathrm{TM}}\right) \varepsilon_{r} \frac{l_{u}}{l_{v}}\left(\frac{\partial}{\partial v} U_{q, \mathrm{TM}} V_{q, \mathrm{TM}}\right)
\end{array}\right) d u d v \\
& =-\frac{1}{k_{\perp, q^{\prime}, \mathrm{TM}} k_{\perp, q, \mathrm{TM}}} \int l_{u} l_{v} U_{q^{\prime}, \mathrm{TM}} V_{q^{\prime}, \mathrm{TM}} \varepsilon_{r} \Delta U_{q, \mathrm{TM}} V_{q, \mathrm{TM}} d u d v-\frac{1}{k_{\perp, q^{\prime}, \mathrm{TM}} k_{\perp, q, \mathrm{TM}}} \int U_{q^{\prime}, \mathrm{TM}} V_{q^{\prime}, \mathrm{TM}}\left(\frac{\partial}{\partial u} \varepsilon_{r}\right) \frac{l_{v}}{l_{u}}\left(\frac{\partial}{\partial u} U_{q, \mathrm{TM}}\right) V_{q, \mathrm{TM}} d u d v \\
& =\frac{k_{\perp, q, \mathrm{TM}}}{k_{\perp, q^{\prime}, \mathrm{TM}}} \int l_{u} l_{v} U_{q^{\prime}, \mathrm{TM}} V_{q^{\prime}, \mathrm{TM}} \varepsilon_{r} U_{q, \mathrm{TM}} V_{q, \mathrm{TM}} d u d v-\frac{1}{k_{\perp, q^{\prime}, \mathrm{TM}} k_{\perp, q, \mathrm{TM}}} \int U_{q^{\prime}, \mathrm{TM}} V_{q^{\prime}, \mathrm{TM}}\left(\frac{\partial}{\partial u} \varepsilon_{r}\right) \frac{l_{v}}{l_{u}}\left(\frac{\partial}{\partial u} U_{q, \mathrm{TM}}\right) V_{q, \mathrm{TM}} d u d v
\end{aligned}
$$

The TETE contribution is

$$
\begin{aligned}
& O_{d e, q^{\prime} q}^{\mathrm{TETE}}=\left\langle\mathbf{g}_{q}^{\prime}, \mathrm{TE}\left|\hat{O}_{d e}\right| \mathbf{g}_{q, \mathrm{TE}}\right\rangle \\
& =\frac{1}{k_{\perp, q, \mathrm{TE}} k_{\perp, q, \mathrm{TE}}} \int l_{u} l_{v}\left(\left[\frac{1}{l_{v}} \frac{\partial}{\partial v}-\frac{1}{l_{u}} \frac{\partial}{\partial u}\right] U_{q^{\prime}, \mathrm{TE}}(u) V_{q^{\prime}, \mathrm{TE}}(v)\right)\left[\begin{array}{cc}
\varepsilon_{r} & 0 \\
0 & \varepsilon_{r}
\end{array}\right]\left[\begin{array}{c}
\frac{1}{l_{v}} \frac{\partial}{\partial v} \\
-\frac{1}{l_{u}} \frac{\partial}{\partial u}
\end{array}\right] U_{q, \mathrm{TE}}(u) V_{q, \mathrm{TE}}(v) d u d v \\
& =\frac{1}{k_{\perp, q, \mathrm{TE}} k_{\perp, q, \mathrm{TE}}} \int\left(\begin{array}{l}
\left(\frac{\partial}{\partial u} U_{q^{\prime}, \mathrm{TE}} V_{q^{\prime}, \mathrm{TE}}\right) \varepsilon_{r} \frac{l_{v}}{l_{u}}\left(\frac{\partial}{\partial u} U_{q, \mathrm{TE}} V_{q, \mathrm{TE}}\right) \\
+\left(\frac{\partial}{\partial v} U_{q^{\prime}, \mathrm{TE}} V_{q^{\prime}, \mathrm{TE}}\right) \varepsilon_{r} \frac{l_{u}}{l_{v}}\left(\frac{\partial}{\partial v} U_{q, \mathrm{TE}} V_{q, \mathrm{TE}}\right)
\end{array}\right) d u d v \\
& =-\frac{1}{k_{\perp, q^{\prime}, \mathrm{TE}} k_{\perp, q, \mathrm{TE}}} \int l_{u} l_{v} U_{q^{\prime}, \mathrm{TE}} V_{q^{\prime}, \mathrm{TE}} \varepsilon_{r} \Delta U_{q, \mathrm{TE}} V_{q, \mathrm{TE}} d u d v-\frac{1}{k_{\perp, q, T E} k_{\perp, q, T E}} \int U_{q^{\prime}, \mathrm{TE}} V_{q^{\prime}, \mathrm{TE}}\left(\frac{\partial}{\partial u} \varepsilon_{r}\right) \frac{l_{v}}{l_{u}}\left(\frac{\partial}{\partial u} U_{q, \mathrm{TE}}\right) V_{q, \mathrm{TE}} d u d v \\
& =\frac{k_{\perp, q, \mathrm{TE}}}{k_{\perp, q^{\prime}, \mathrm{TE}}} \int l_{u} l_{v} U_{q^{\prime}, \mathrm{TE}} V_{q^{\prime}, \mathrm{TE}} \varepsilon_{r} U_{q, \mathrm{TE}} V_{q, \mathrm{TE}} d u d v-\frac{1}{k_{\perp, q^{\prime}, \mathrm{TE}} k_{\perp, q, \mathrm{TE}}} \int U_{q^{\prime}, \mathrm{TE}} V_{q^{\prime}, \mathrm{TE}}\left(\frac{\partial}{\partial u} \varepsilon_{r}\right) \frac{l_{v}}{l_{u}}\left(\frac{\partial}{\partial u} U_{q, \mathrm{TE}}\right) V_{q, \mathrm{TE}} d u d v
\end{aligned}
$$

The TMTE element of the $\overline{\bar{O}}_{d e}$ matrix is given by

$$
\begin{aligned}
& O_{d e, q^{\prime} q}^{\mathrm{TMTE}}=\left\langle\mathbf{f}_{q, \mathrm{TM}}\left|\hat{O}_{d e}\right| \mathbf{g}_{q, \mathrm{TE}}\right\rangle \\
& =\frac{1}{k_{\perp, q^{\prime}, \mathrm{TM}} k_{\perp, q, \mathrm{TE}}} \int l_{u} l_{v}\left(\left[\begin{array}{ll}
\frac{1}{l_{u}} \frac{\partial}{\partial u} & \frac{1}{l_{v}} \frac{\partial}{\partial v}
\end{array}\right] U_{q^{\prime}, \mathrm{TM}}(u) V_{q^{\prime}, \mathrm{TM}}(v)\right)\left[\begin{array}{cc}
\varepsilon_{r} & 0 \\
0 & \varepsilon_{r}
\end{array}\right]\left[\begin{array}{c}
\frac{1}{l_{v}} \frac{\partial}{\partial v} \\
-\frac{1}{l_{u}} \frac{\partial}{\partial u}
\end{array}\right] U_{q, \mathrm{TE}}(u) V_{q, \mathrm{TE}}(v) d u d v \\
& =\frac{1}{k_{\perp, q^{\prime}, \mathrm{TM}} k_{\perp, q, \mathrm{TE}}} \int\left(\begin{array}{c}
\left(\frac{\partial}{\partial u} U_{q^{\prime}, \mathrm{TM}} V_{q^{\prime}, \mathrm{TM}}\right) \varepsilon_{r}\left(\frac{\partial}{\partial v} U_{q, \mathrm{TE}} V_{q, \mathrm{TE}}\right) \\
-\left(\frac{\partial}{\partial v} U_{q^{\prime}, \mathrm{TM}} V_{q^{\prime}, \mathrm{TM}}\right) \varepsilon_{r}\left(\frac{\partial}{\partial u} U_{q, \mathrm{TE}} V_{q, \mathrm{TE}}\right)
\end{array}\right) d u d v \\
& =-\frac{1}{k_{\perp, q^{\prime}, \mathrm{TM}} k_{\perp, q, \mathrm{TE}}} \int U_{q^{\prime}, \mathrm{TM}} V_{q^{\prime}, \mathrm{TM}}\left(\frac{\partial}{\partial u} \varepsilon_{r}\right) U_{q, \mathrm{TE}}\left(\frac{\partial}{\partial v} V_{q, \mathrm{TE}}\right) d u d v
\end{aligned}
$$

and finally the TETM element of the $\overline{\overline{O_{d e}}}$ matrix is 


$$
\begin{aligned}
& O_{d e, q^{\prime} q}^{\mathrm{TETM}}=\left\langle\mathbf{g}_{q^{\prime} ; \mathrm{TE}}\left|\hat{O}_{d e}\right| \mathbf{f}_{q, \mathrm{TM}}\right\rangle \\
& =\frac{1}{k_{\perp, q^{\prime}, \mathrm{TE}} k_{\perp, q, \mathrm{TM}}} \int l_{u} l_{v}\left(\left[\frac{1}{l_{v}} \frac{\partial}{\partial v}-\frac{1}{l_{u}} \frac{\partial}{\partial u}\right] U_{q^{\prime}, \mathrm{TE}}(u) V_{q^{\prime}, \mathrm{TE}}(v)\right)\left[\begin{array}{cc}
\varepsilon_{r} & 0 \\
0 & \varepsilon_{r}
\end{array}\right]\left[\begin{array}{c}
\frac{1}{l_{u}} \frac{\partial}{\partial u} \\
\frac{1}{l_{v}} \frac{\partial}{\partial v}
\end{array}\right] U_{q, \mathrm{TM}}(u) V_{q, \mathrm{TM}}(v) d u d v \\
& =\frac{1}{k_{\perp, q^{\prime}, \mathrm{TE}} k_{\perp, q, \mathrm{TM}}} \int\left(\begin{array}{l}
-\left(\frac{\partial}{\partial u} U_{q^{\prime}, \mathrm{TE}} V_{q^{\prime}, \mathrm{TE}}\right) \varepsilon_{r}\left(\frac{\partial}{\partial v} U_{q, \mathrm{TM}} V_{q, \mathrm{TM}}\right) \\
+\left(\frac{\partial}{\partial v} U_{q^{\prime}, \mathrm{TE}} V_{q^{\prime}, \mathrm{TE}}\right) \varepsilon_{r}\left(\frac{\partial}{\partial u} U_{q, \mathrm{TM}} V_{q, \mathrm{TM}}\right)
\end{array}\right) d u d v \\
& =\frac{1}{k_{\perp, q, \mathrm{TE}} k_{\perp, q, \mathrm{TM}}} \int U_{q^{\prime}, \mathrm{TE}} V_{q^{\prime}, \mathrm{TE}}\left(\frac{\partial}{\partial u} \varepsilon_{r}\right) U_{q, \mathrm{TM}}\left(\frac{\partial}{\partial v} V_{q, \mathrm{TM}}\right) d u d v
\end{aligned}
$$

\subsection{Calculation of $\mathrm{z}$ field components}

The $z$ components of the fields can be written in matrix form as

$$
\begin{aligned}
& e_{z}(u, v)=\sum_{q} c_{q}^{E_{z}} \Psi_{q, \mathrm{TM}}(u, v)=\bar{\Psi}_{\mathrm{TM}}^{T} \bar{c}^{E_{z}} \\
& h_{z}(u, v)=\sum_{q} c_{q}^{H_{z}} \Psi_{q, \mathrm{TE}}(u, v)=\bar{\Psi}_{\mathrm{TE}}^{T} \bar{c}^{H_{z}}
\end{aligned}
$$

and they can be computed using (73)-(74), which can be written in operator form as

$$
\begin{aligned}
& \hat{O}_{E_{z} H}\left[\begin{array}{l}
h_{u} \\
h_{v}
\end{array}\right]=e_{z} \\
& \hat{O}_{H_{z} E}\left[\begin{array}{l}
e_{u} \\
e_{v}
\end{array}\right]=h_{z},
\end{aligned}
$$

where

$$
\begin{gathered}
\hat{O}_{E_{z} H}=i\left[\begin{array}{cc}
-\frac{1}{k_{0}^{2} \varepsilon_{r}} \frac{1}{l_{u} l_{v}} \frac{\partial}{\partial v} l_{u} & \frac{1}{k_{0}^{2} \varepsilon_{r}} \frac{1}{l_{u} l_{v}} \frac{\partial}{\partial u} l_{v}
\end{array}\right] \\
\hat{O}_{H_{z} E}=i\left[\begin{array}{ll}
\frac{1}{l_{u} l_{v}} \frac{\partial}{\partial v} l_{u} & -\frac{1}{l_{u} l_{v}} \frac{\partial}{\partial u} l_{v}
\end{array}\right]
\end{gathered}
$$

Using the field expansions (57), (58), (80) and (81) we can write (111)-(112) as

$$
\begin{gathered}
\hat{O}_{E_{z} H}\left[\begin{array}{ll}
\bar{g}_{u, \mathrm{TM}}^{T} & \bar{f}_{u, \mathrm{TE}}^{T} \\
\bar{g}_{v, \mathrm{TM}}^{T} & \bar{f}_{v, \mathrm{TE}}^{T}
\end{array}\right]\left[\begin{array}{c}
-H_{\perp} \\
c_{\mathrm{TM}} \\
-H_{\perp} \\
\overline{\mathrm{T}}_{\mathrm{TE}}
\end{array}\right]=\bar{\Psi}_{\mathrm{TM}}^{T} \bar{c}^{E_{z}} \\
\hat{O}_{H_{z} E}\left[\begin{array}{ll}
\bar{f}_{u, \mathrm{TM}}^{T} & \bar{g}_{u, \mathrm{TE}}^{T} \\
\bar{f}_{v, \mathrm{TM}}^{T} & \bar{g}_{v, \mathrm{TE}}^{T}
\end{array}\right]\left[\begin{array}{l}
-E_{\perp} \\
\bar{c}_{\mathrm{TM}} \\
-E_{\perp} \\
c_{\mathrm{TE}}
\end{array}\right]=\bar{\Psi}_{\mathrm{TE}}^{T} \bar{c}^{H_{z}} .
\end{gathered}
$$

It turns out that only the $\bar{c}_{\mathrm{TM}}$ terms contribute to the $E_{z}$ field and only the $\bar{c}_{\mathrm{TE}}$ terms contribute to the $H_{z}$ field. To see this, let us write 


$$
\begin{aligned}
& \hat{O}_{E_{z} H}\left[\begin{array}{ll}
g_{u, q, \mathrm{TM}} & f_{u, q, \mathrm{TE}} \\
g_{v, q, \mathrm{TM}} & f_{v, q, \mathrm{TE}}
\end{array}\right]=i\left[\begin{array}{ll}
-\frac{1}{k_{0}^{2} \varepsilon_{r}} \frac{1}{l_{u} l_{v}} \frac{\partial}{\partial v} l_{u} & \frac{1}{k_{0}^{2} \varepsilon_{r}} \frac{1}{l_{u} l_{v}} \frac{\partial}{\partial u} l_{v}
\end{array}\right]\left[\begin{array}{ll}
-\frac{1}{k_{\perp, q, \mathrm{TM}} l_{v}} \frac{\partial}{\partial v} U_{q, \mathrm{TM}} V_{q, \mathrm{TM}} & \frac{1}{k_{\perp, q, \mathrm{TE}} l_{u}} \frac{\partial}{\partial u} U_{q, \mathrm{TE}} V_{q, \mathrm{TE}} \\
\frac{1}{k_{\perp, q, \mathrm{TM}} l_{u}} \frac{\partial}{\partial u} U_{q, \mathrm{TM}} V_{q, \mathrm{TM}} & \frac{1}{k_{\perp, q, \mathrm{TE}} l_{v}} \frac{\partial}{\partial v} U_{q, \mathrm{TE}} V_{q, \mathrm{TE}}
\end{array}\right] \\
& =\frac{i}{k_{0}^{2} \varepsilon_{r}}\left[\frac{1}{k_{\perp, q, \mathrm{TM}}} \Delta U_{q, \mathrm{TM}} V_{q, \mathrm{TM}} \quad 0\right] \\
& \hat{O}_{H_{z} E}\left[\begin{array}{ll}
f_{u, q, \mathrm{TM}} & g_{u, q, \mathrm{TE}} \\
f_{v, q, \mathrm{TM}} & g_{v, q, \mathrm{TE}}
\end{array}\right]=i\left[\begin{array}{lll}
\frac{1}{l_{u} l_{v}} \frac{\partial}{\partial v} l_{u} & -\frac{1}{l_{u} l_{v}} \frac{\partial}{\partial u} l_{v}
\end{array}\right]\left[\begin{array}{cc}
\frac{1}{k_{\perp, q, \mathrm{TM}} l_{u}} \frac{\partial}{\partial u} U_{q, \mathrm{TM}} V_{q, \mathrm{TM}} & \frac{1}{k_{\perp, q, \mathrm{TE}} l_{v}} \frac{\partial}{\partial v} U_{q, \mathrm{TE}} V_{q, \mathrm{TE}} \\
\frac{1}{k_{\perp, q, \mathrm{TM}} l_{v}} \frac{\partial}{\partial v} U_{q, \mathrm{TM}} V_{q, \mathrm{TM}} & -\frac{1}{k_{\perp, q, \mathrm{TE}} l_{u}} \frac{\partial}{\partial u} U_{q, \mathrm{TE}} V_{q, \mathrm{TE}}
\end{array}\right] . \\
& =i\left[\begin{array}{ll}
0 & \left.\frac{1}{k_{\perp, q, \mathrm{TE}}} \Delta U_{q, \mathrm{TE}} V_{q, \mathrm{TE}}\right]
\end{array}\right.
\end{aligned}
$$

Thus, by multiplying (115) with $\int \bar{\Psi}_{\mathrm{TM}} d \mathbf{r}_{\perp}$ and (116) with $\int \bar{\Psi}_{\mathrm{TE}} d \mathbf{r}_{\perp}$ and exploiting the orthogonality relation (14) and (117)-(118), we obtain

$$
\begin{aligned}
& \bar{c}^{E_{z}}=\overline{\bar{O}}_{E_{z} H} \bar{c}_{\mathrm{TM}}^{H_{\perp}} \\
& \bar{c}^{H_{z}}=\overline{\bar{O}}_{H_{z} E} \bar{c}_{\mathrm{TE}},
\end{aligned}
$$

where the matrix elements are given by

$$
\begin{gathered}
O_{E_{z} H, q^{\prime} q}=\left\langle\Psi_{q^{\prime}, \mathrm{TM}}\left|\frac{i}{k_{0}^{2} \varepsilon_{r} k_{\perp, q, \mathrm{TM}}} \Delta\right| \Psi_{q, \mathrm{TM}}\right\rangle=-\left\langle\Psi_{q^{\prime}, \mathrm{TM}}\left|\frac{i k_{\perp, q, \mathrm{TM}}}{k_{0}^{2} \varepsilon_{r}}\right| \Psi_{q, \mathrm{TM}}\right\rangle \\
=-i k_{\perp, q, \mathrm{TM}} \int U_{q^{\prime}, \mathrm{TM}} V_{q^{\prime}, \mathrm{TM}} \frac{1}{k_{0}^{2} \varepsilon_{r}} U_{q, \mathrm{TM}} V_{q, \mathrm{TM}} l_{u} l_{v} d u d v \\
O_{H_{z} E, q^{\prime} q}=i\left\langle\Psi_{q^{\prime}, \mathrm{TE}}\left|\frac{1}{k_{\perp, q, \mathrm{TE}}} \Delta\right| \Psi_{q, \mathrm{TE}}\right\rangle=-i\left\langle\Psi_{q^{\prime}, \mathrm{TE}}\left|k_{\perp, q, \mathrm{TE}}\right| \Psi_{q, \mathrm{TE}}\right\rangle \\
=-i k_{\perp, q, \mathrm{TE}} \int U_{q^{\prime}, \mathrm{TE}} V_{q^{\prime}, \mathrm{TE}} U_{q, \mathrm{TE}} V_{q, \mathrm{TE}} l_{u} l_{v} d u d v=-i k_{\perp, q, \mathrm{TE}} \delta_{q^{\prime} q}
\end{gathered}
$$

\subsection{Orthonormalization of eigenmodes}

Using the Lorentz reciprocity theorem, it can be proven [1] that the eigenmodes with different propagation constants are orthogonal to each other with respect to the product

$$
\int\left(\mathbf{e}_{j} \times \mathbf{h}_{j^{\prime}}\right) \cdot \mathbf{z} d \mathbf{r}_{\perp}=0 \quad\left(j \neq j^{\prime}\right),
$$

where $\mathbf{z}$ is the unit vector along the $z$ axis and $d \mathbf{r}_{\perp}=d x d y=l_{u} l_{v} d u d v$. A possible normalization condition is thus the following:

$$
\int\left(\mathbf{e}_{j} \times \mathbf{h}_{j^{\prime}}\right) \cdot \mathbf{z} d \mathbf{r}_{\perp}=\delta_{i j^{\prime}}
$$

The integral looks very much like a calculation of the power flowing through the plane by integration of the Poynting vector, but there is a subtle difference. For our harmonic time dependence, the power $P$ flowing through the plane is

$$
P=\frac{1}{2} \operatorname{Re}\left(\int \mathbf{S} \cdot \mathbf{z} d \mathbf{r}_{\perp}\right)=\frac{1}{2} \operatorname{Re}\left(\int\left(\mathbf{e}^{*} \times \mathbf{h}\right) \cdot \mathbf{z} d \mathbf{r}_{\perp}\right),
$$

where the electrical field has been complex conjugated. It turns out that the orthogonality condition 


$$
\int\left(\mathbf{e}_{j}^{*} \times \mathbf{h}_{j^{\prime}}\right) \cdot \mathbf{z} d \mathbf{r}_{\perp}=0 \quad\left(j \neq j^{\prime}\right)
$$

is also valid, but it only holds for a real-valued refractive index profile. On the other hand, the orthogonality condition (123) also holds for a complex-valued refractive index profile, which is relevant in the presence of loss or gain, and is thus more general. For this reason, we usually work with the orthonormalization condition (123) instead of (126). The normalization (126) is sometimes referred to as "power normalization".

To normalize the eigenmode $j$, we compute the integral

$$
\int\left(\mathbf{e}_{j} \times \mathbf{h}_{j}\right) \cdot \mathbf{z} d \mathbf{r}_{\perp}=\int\left(\mathbf{e}_{\perp, j} \times \mathbf{h}_{\perp, j}\right) \cdot \mathbf{z} l_{u} l_{v} d u d v=N_{j}
$$

by inserting the expansions (54)-(55) into (127). Using the relations

$$
\begin{gathered}
\int\left(\mathbf{f}_{q^{\prime}, \mathrm{TM}} \times \mathbf{g}_{q, \mathrm{TM}}\right) \cdot \mathbf{z} d \mathbf{r}_{\perp}=\left\langle\mathbf{f}_{q^{\prime}, \mathrm{TM}} \mid \mathbf{f}_{q, \mathrm{TM}}\right\rangle=\delta_{q^{\prime} q} \\
\int\left(\mathbf{g}_{q^{\prime}, \mathrm{TE}} \times \mathbf{f}_{q, \mathrm{TE}}\right) \cdot \mathbf{z} d \mathbf{r}_{\perp}=\left\langle\mathbf{g}_{q^{\prime}, \mathrm{TE}} \mid \mathbf{g}_{q, \mathrm{TE}}\right\rangle=\delta_{q^{\prime} q} \\
\int\left(\mathbf{f}_{q^{\prime}, \mathrm{TM}} \times \mathbf{f}_{q, \mathrm{TE}}\right) \cdot \mathbf{z} d \mathbf{r}_{\perp}=\left\langle\mathbf{f}_{q^{\prime}, \mathrm{TM}} \mid \mathbf{g}_{q, \mathrm{TE}}\right\rangle=0 \\
\int\left(\mathbf{g}_{q^{\prime}, \mathrm{TE}} \times \mathbf{g}_{q, \mathrm{TM}}\right) \cdot \mathbf{z} d \mathbf{r}_{\perp}=\left\langle\mathbf{g}_{q^{\prime}, \mathrm{TE}} \mid \mathbf{f}_{q, \mathrm{TM}}\right\rangle=0,
\end{gathered}
$$

we obtain

$$
\int\left(\mathbf{e}_{\perp, j} \times \mathbf{h}_{\perp, j}\right) \cdot \mathbf{z} l_{u} l_{v} d u d v=\sum_{q}\left(c_{\mathrm{TM}, q j}^{E_{\perp}} c_{\mathrm{TM}, q j}^{H_{\perp}}+c_{\mathrm{TE}, q j}^{E_{\perp}} c_{\mathrm{TE}, q j}^{H_{\perp}}\right)=N_{j} .
$$

The normalization is then obtained by performing the replacement

$$
\left(\overline{c_{\mathrm{TM}, j}^{E_{\perp}}}, \overline{c_{\mathrm{TE}, j}^{E_{\perp}}}, \overline{c_{\mathrm{TM}, j}^{H_{\perp}}}, \overline{c_{\mathrm{TE}, j}^{H_{\perp}}}\right) \rightarrow\left(\overline{c_{\mathrm{TM}, j}^{E_{\perp}}}, \overline{c_{\mathrm{TE}, j}^{E_{\perp}}}, \overline{c_{\mathrm{TM}, j}^{H_{\perp}}}, \overline{c_{\mathrm{TE}, j}^{H_{\perp}}}\right) / \sqrt{N_{j}} .
$$

Similarly, we can compute the power $P_{j}$ carried by a mode $j$ as

$$
P_{j}=\frac{1}{2} \operatorname{Re}\left(\int\left(\mathbf{e}_{j}^{*} \times \mathbf{h}_{j}\right) \cdot \mathbf{z} d \mathbf{r}_{\perp}\right)=\frac{1}{2} \operatorname{Re}\left(\sum_{q}\left(c_{\mathrm{TM}, q j}^{E_{\perp}}{ }^{*} c_{\mathrm{TM}, q j}^{H_{\perp}}+c_{\mathrm{TE}, q j}^{E_{\perp}}{ }^{*} c_{\mathrm{TE}, q j}^{H_{\perp}}\right)\right),
$$

where we have exploited that our basis modes are real-valued.

\section{Open geometry}

We now consider an open geometry along the $u$ axis, where the computational domain is contained in the intervals $0<u<\infty$ and $0 \leq v \leq 2 \pi$. We assume that the relative permittivity is of the form $\varepsilon_{r}(u)=\varepsilon_{r, \mathrm{~B}}+\Delta \varepsilon_{r}(u)$, where $\Delta \varepsilon_{r}(u)=0$ for $u>u_{\mathrm{B}}$. Similarly, the relative impermittivity (the inverse of the relative permittivity) is given by $\eta_{r}(u)=1 / \varepsilon_{r}(u)=\eta_{r, \mathrm{~B}}+\Delta \eta_{r}(u)$, where $\Delta \eta_{r}(u)=0$ for $u>u_{\mathrm{B}}$. Our basis functions are now written as $\Psi\left(k_{\perp}, m, \mathbf{r}_{\perp}\right)=U\left(k_{\perp}, m, u\right) V\left(k_{\perp}, m, v\right)$. They are indexed by the continuous transverse wavenumber $k_{\perp}$ and a discrete angular order $m$, where we in the following use the $k_{\perp}$ index for both $k_{\perp}$ and $m$ for simplicity. The normalization condition for the basis functions is now given by

$$
\int_{u=0, v=0}^{u=\infty, v=2 \pi} U\left(k^{\prime}{ }_{\perp}, u\right) V\left(k^{\prime}{ }_{\perp}, v\right) U\left(k_{\perp}, u\right) V\left(k_{\perp}, v\right) l_{u} l_{v} d u d v=\delta\left(k^{\prime}{ }_{\perp}-k_{\perp}\right),
$$

where $\delta\left(k^{\prime}{ }_{\perp}-k_{\perp}\right)$ is the Dirac delta function. As for the closed geometry, once suitable functions $U$ and $V$ have been identified, the normalization can be established by computing the integral 


$$
\int_{u=0, v=0}^{u=\infty, v=2 \pi} U\left(k^{\prime}{ }_{\perp}, u\right) V\left(k^{\prime}{ }_{\perp}, v\right) U\left(k_{\perp}, u\right) V\left(k_{\perp}, v\right) l_{u} l_{v} d u d v=N\left(k_{\perp}\right) \delta\left(k^{\prime}{ }_{\perp}-k_{\perp}\right) .
$$

and then performing the replacement $U\left(k_{\perp}, u\right) V\left(k_{\perp}, v\right) \rightarrow U\left(k_{\perp}, u\right) V\left(k_{\perp}, v\right) / \sqrt{N\left(k_{\perp}\right)}$.

We will use the same basis modes (49)-(53) as before, where the discrete $k_{\perp, q, \mathrm{TX}}$ values defined by the closed boundary condition in Section 2.2 is now replaced by a continuous range $k_{\perp, \mathrm{TX}}$. For the open boundary geometry, we will use the same continuous set of $k_{\perp, \mathrm{TX}}$ values ranging from 0 to $\infty$ for both TE and TM modes, and the TX index is thus no longer required for the in-plane $k$ vector amplitude. The orthonormalization conditions for the basis modes become

$$
\begin{aligned}
& \left\langle\mathbf{f}_{\mathrm{TX}}\left(k_{\perp}^{\prime}\right) \mid \mathbf{f}_{\mathrm{TX}}\left(k_{\perp}\right)\right\rangle=\left\langle\mathbf{g}_{\mathrm{TX}}\left(k_{\perp}^{\prime}\right) \mid \mathbf{g}_{\mathrm{TX}}\left(k_{\perp}\right)\right\rangle \\
& =\frac{1}{k_{\perp}^{\prime} k_{\perp}} \int\left[\left(\frac{\partial}{\partial u} U_{\mathrm{TX}}\left(k_{\perp}^{\prime}\right) V_{\mathrm{TX}}\left(k_{\perp}^{\prime}\right)\right) \frac{l_{v}}{l_{u}}\left(\frac{\partial}{\partial u} U_{\mathrm{TX}}\left(k_{\perp}\right) V_{\mathrm{TX}}\left(k_{\perp}\right)\right)+\left(\frac{\partial}{\partial v} U_{\mathrm{TX}}\left(k_{\perp}^{\prime}\right) V_{\mathrm{TX}}\left(k_{\perp}^{\prime}\right)\right) \frac{l_{u}}{l_{v}}\left(\frac{\partial}{\partial v} U_{\mathrm{TX}}\left(k_{\perp}\right) V_{\mathrm{TX}}\left(k_{\perp}\right)\right)\right] d u d v, \\
& =-\frac{1}{k_{\perp}^{\prime} k_{\perp}} \int\left[\left(\frac{\partial}{\partial u} \frac{l_{v}}{l_{u}} \frac{\partial}{\partial u} U_{\mathrm{TX}}\left(k_{\perp}^{\prime}\right) V_{\mathrm{TX}}\left(k_{\perp}^{\prime}\right)\right) U_{\mathrm{TX}}\left(k_{\perp}\right) V_{\mathrm{TX}}\left(k_{\perp}\right)+\left(\frac{\partial}{\partial v} \frac{l_{u}}{l_{v}} \frac{\partial}{\partial v} U_{\mathrm{TX}}\left(k_{\perp}^{\prime}\right) V_{\mathrm{TX}}\left(k_{\perp}^{\prime}\right)\right) U_{\mathrm{TX}}\left(k_{\perp}\right) V_{\mathrm{TX}}\left(k_{\perp}\right)\right] d u d v \\
& =-\frac{1}{k_{\perp}^{\prime} k_{\perp}} \int l_{v} l_{u}\left(\Delta U_{\mathrm{TX}}\left(k_{\perp}^{\prime}\right) V_{\mathrm{TX}}\left(k_{\perp}^{\prime}\right)\right) U_{\mathrm{TX}}\left(k_{\perp}\right) V_{\mathrm{TX}}\left(k_{\perp}\right) d u d v=\frac{k_{\perp}^{\prime}}{k_{\perp}} \int l_{v} l_{u} U_{\mathrm{TX}}\left(k_{\perp}^{\prime}\right) V_{\mathrm{TX}}\left(k_{\perp}^{\prime}\right) U_{\mathrm{TX}}\left(k_{\perp}\right) V_{\mathrm{TX}}\left(k_{\perp}\right) d u d v=\delta\left(k_{\perp}^{\prime}-k_{\perp}\right)
\end{aligned}
$$

where we used integration by parts in the second step. Here and in the following we assume that $\lim _{u \rightarrow \infty} U\left(k_{\perp}, u\right)=0$, as it is the case for the rotationally symmetric and the elliptical geometries, such that the boundary term for $u=\infty$ in the integrations by parts in Eqs. (17)-(18) vanishes.

And, as before, we have

$$
\begin{aligned}
& \left\langle\mathbf{f}_{\mathrm{TX}}\left(k_{\perp}^{\prime}\right) \mid \mathbf{g}_{\mathrm{TY}}\left(k_{\perp}\right)\right\rangle=\left\langle\mathbf{g}_{\mathrm{TX}}\left(k_{\perp}\right) \mid \mathbf{f}_{\mathrm{TY}}\left(k_{\perp}^{\prime}\right)\right\rangle \\
& =\frac{1}{k_{\perp}^{\prime} k_{\perp}} \int\left[\left(\frac{\partial}{\partial u} U_{\mathrm{TX}}\left(k_{\perp}^{\prime}\right) V_{\mathrm{TX}}\left(k_{\perp}^{\prime}\right)\right)\left(\frac{\partial}{\partial v} U_{\mathrm{TY}}\left(k_{\perp}\right) V_{\mathrm{TY}}\left(k_{\perp}\right)\right)-\left(\frac{\partial}{\partial v} U_{\mathrm{TX}}\left(k_{\perp}^{\prime}\right) V_{\mathrm{TX}}\left(k_{\perp}^{\prime}\right)\right)\left(\frac{\partial}{\partial u} U_{\mathrm{TY}}\left(k_{\perp}\right) V_{\mathrm{TY}}\left(k_{\perp}\right)\right)\right] d u d v . \\
& =-\frac{1}{k_{\perp}^{\prime} k_{\perp}} \int\left[\left(\frac{\partial}{\partial v} \frac{\partial}{\partial u} U_{\mathrm{TX}}\left(k_{\perp}^{\prime}\right) V_{\mathrm{TX}}\left(k_{\perp}^{\prime}\right)\right) U_{\mathrm{TY}}\left(k_{\perp}\right) V_{\mathrm{TY}}\left(k_{\perp}\right)-\left(\frac{\partial}{\partial u} \frac{\partial}{\partial v} U_{\mathrm{TX}}\left(k_{\perp}^{\prime}\right) V_{\mathrm{TX}}\left(k_{\perp}^{\prime}\right)\right) U_{\mathrm{TY}}\left(k_{\perp}\right) V_{\mathrm{TY}}\left(k_{\perp}\right)\right] d u d v=0
\end{aligned}
$$

The $j$ 'th eigenmode is then given as

$$
\begin{aligned}
& \mathbf{e}_{\perp, j}(u, v)=\left[\begin{array}{l}
e_{u, j}(u, v) \\
e_{v, j}(u, v)
\end{array}\right]=\int c_{\mathrm{TM}, j}^{E_{\perp}}\left(k_{\perp}\right) \mathbf{f}_{\mathrm{TM}}\left(k_{\perp}, u, v\right) d q+\int c_{\mathrm{TE}, j}^{E_{\perp}}\left(k_{\perp}\right) \mathbf{g}_{\mathrm{TE}}\left(k_{\perp}, u, v\right) d q=\int\left[\begin{array}{ll}
f_{u, \mathrm{TM}}\left(k_{\perp}\right) & g_{u, \mathrm{TE}}\left(k_{\perp}\right) \\
f_{v, \mathrm{TM}}\left(k_{\perp}\right) & g_{v, \mathrm{TE}}\left(k_{\perp}\right)
\end{array}\right]\left[\begin{array}{l}
c_{\mathrm{TM}}^{E_{\perp}}\left(k_{\perp}\right) \\
c_{\mathrm{TE}}^{E_{\perp}}\left(k_{\perp}\right)
\end{array}\right] d k_{\perp} \\
& \mathbf{h}_{\perp, j}(u, v)=\left[\begin{array}{l}
h_{u, j}(u, v) \\
h_{v, j}(u, v)
\end{array}\right]=\int c_{\mathrm{TM}, j}^{H_{\perp}}\left(k_{\perp}\right) \mathbf{g}_{\mathrm{TM}}\left(k_{\perp}, u, v\right) d q+\int c_{\mathrm{TE}, j}^{H_{\perp}}\left(k_{\perp}\right) \mathbf{f}_{\mathrm{TE}}\left(k_{\perp}, u, v\right) d q=\int\left[\begin{array}{ll}
g_{u, \mathrm{TM}}\left(k_{\perp}\right) & f_{u, \mathrm{TE}}\left(k_{\perp}\right) \\
g_{v, \mathrm{TM}}\left(k_{\perp}\right) & f_{v, \mathrm{TE}}\left(k_{\perp}\right)
\end{array}\right]\left[\begin{array}{c}
c_{\mathrm{TM}}^{H_{\perp}}\left(k_{\perp}\right) \\
c_{\mathrm{TE}}^{H_{\perp}}\left(k_{\perp}\right)
\end{array}\right] d k_{\perp} \\
& e_{z, j}(u, v)=\int c_{j}^{E_{z}}\left(k_{\perp}\right) \Psi_{\mathrm{TM}}\left(k_{\perp}, u, v\right) d q=\int c_{j}^{E_{z}}\left(k_{\perp}\right) U_{\mathrm{TM}}\left(k_{\perp}, u\right) V_{\mathrm{TM}}\left(k_{\perp}, v\right) d k_{\perp} \\
& h_{z, j}(u, v)=\int c_{j}^{H_{z}}\left(k_{\perp}\right) \Psi_{\mathrm{TE}}\left(k_{\perp}, u, v\right) d q=\int c_{j}^{H_{z}}\left(k_{\perp}\right) U_{\mathrm{TE}}\left(k_{\perp}, u\right) V_{\mathrm{TE}}\left(k_{\perp}, v\right) d q,
\end{aligned}
$$

where the sums from the closed geometry description have been replaced by integrals.

Our strategy for handling the open geometry will be to transform integrals over the continuous index $k_{\perp}$ into Riemann sums as

$$
\int_{0}^{\infty} c\left(k_{\perp}\right) f\left(k_{\perp}\right) d k_{\perp} \rightarrow \sum_{q} c_{q} f\left(k_{\perp, q}\right) \Delta k_{\perp, q}
$$

with the shorthand notation

$$
c\left(k_{\perp}\right) \rightarrow c\left(k_{\perp, q}\right)=c_{q},
$$


and where we allow for a non-uniform sampling of the $k_{\perp}$ space by introducing a non-equidistant $q$ dependent discretization width $\Delta k_{\perp, q}$ in the Riemann sum. With this notation, the eigenmode $j$ fields are then given by

$$
\begin{aligned}
& \mathbf{e}_{\perp, j}(u, v)= {\left[\begin{array}{l}
e_{u, j}(u, v) \\
e_{v, j}(u, v)
\end{array}\right]=\sum_{q} c_{\mathrm{TM}, q j}^{E_{\perp}} \mathbf{f}_{\mathrm{TM}}\left(k_{\perp, q}, u, v\right) \Delta k_{\perp, q}+\sum_{m} c_{\mathrm{TE}, q j}^{E_{\perp}} \mathbf{g}_{\mathrm{TE}}\left(k_{\perp, q}, u, v\right) \Delta k_{\perp, q} } \\
& \mathbf{h}_{\perp, j}(u, v)= {\left[\begin{array}{l}
h_{u, j}(u, v) \\
h_{v, j}(u, v)
\end{array}\right]=\sum_{q} c_{\mathrm{TM}, q j}^{H_{\perp}} \mathbf{g}_{\mathrm{TM}}\left(k_{\perp, q}, u, v\right) \Delta k_{\perp, q}+\sum_{q} c_{\mathrm{TE}, q j}^{H_{\perp}} \mathbf{f}_{\mathrm{TE}}\left(k_{\perp, q}, u, v\right) \Delta k_{\perp, q} } \\
& \mathbf{d}_{\perp, j}(u, v)=\left[\begin{array}{l}
d_{u, j}(u, v) \\
d_{v, j}(u, v)
\end{array}\right]=\sum_{q} c_{\mathrm{TM}, q j}^{D_{\perp}} \mathbf{f}_{\mathrm{TM}}\left(k_{\perp, q}, u, v\right) \Delta k_{\perp, q}+\sum_{q} c_{\mathrm{TE}, q j}^{D_{\perp}} \mathbf{g}_{\mathrm{TE}}\left(k_{\perp, q}, u, v\right) \Delta k_{\perp, q} \\
& e_{z, j}(u, v)=\sum_{q} c_{q j}^{E_{z}} \Psi_{\mathrm{TM}}\left(k_{\perp, q}, u, v\right) \Delta k_{\perp, q} \\
& h_{z, j}(u, v)=\sum_{q} c_{q j}^{H_{z}} \Psi_{\mathrm{TE}}\left(k_{\perp, q}, u, v\right) \Delta k_{\perp, q} .
\end{aligned}
$$

The operator Eq. (76) can be written as

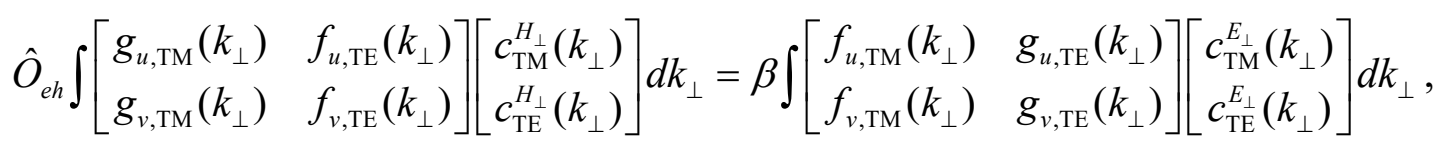

where we have not yet implemented the Riemann sum.

For the open geometry, the orthonormalization condition gives

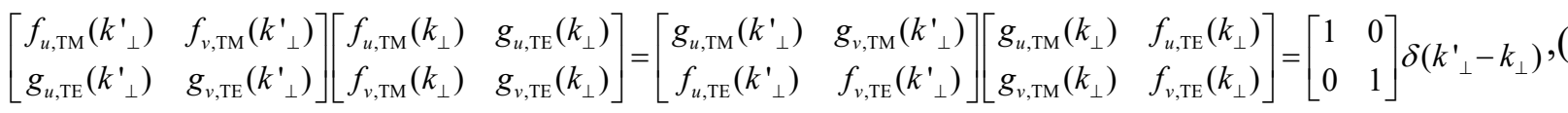

and by multiplying (150) with $\int\left[\begin{array}{ll}f_{u, \mathrm{TM}}\left(k_{\perp}^{\prime}\right) & f_{v, \mathrm{TM}}\left(k_{\perp}^{\prime}\right) \\ g_{u, \mathrm{TE}}\left(k_{\perp}^{\prime}\right) & g_{v, \mathrm{TE}}\left(k_{\perp}^{\prime}\right)\end{array}\right] d \mathbf{r}_{\perp}$, we obtain

$$
\int\left[\begin{array}{cc}
f_{u, \mathrm{TM}}\left(k_{\perp}^{\prime}\right) & f_{v, \mathrm{TM}}\left(k_{\perp}^{\prime}\right) \\
g_{u, \mathrm{TE}}\left(k_{\perp}^{\prime}\right) & g_{v, \mathrm{TE}}\left(k_{\perp}^{\prime}\right)
\end{array}\right] \hat{O}_{e h}\left[\begin{array}{ll}
g_{u, \mathrm{TM}}\left(k_{\perp}\right) & f_{u, \mathrm{TE}}\left(k_{\perp}\right) \\
g_{v, \mathrm{TM}}\left(k_{\perp}\right) & f_{v, \mathrm{TE}}\left(k_{\perp}\right)
\end{array}\right]\left[\begin{array}{c}
c_{\mathrm{TM}}^{H_{\perp}}\left(k_{\perp}\right) \\
c_{\mathrm{TE}}^{H_{\perp}}\left(k_{\perp}\right)
\end{array}\right] d k_{\perp} d \mathbf{r}_{\perp}=\beta\left[\begin{array}{c}
c_{\mathrm{TM}}^{E_{\perp}}\left(k^{\prime}{ }_{\perp}\right) \\
c_{\mathrm{TE}}^{E_{\perp}}\left(k_{\perp}^{\prime}\right)
\end{array}\right] .
$$

This can be written as

$$
\int \overline{\bar{O}}_{e h}\left(k_{\perp}^{\prime}, k_{\perp}\right)\left[\begin{array}{c}
c_{\mathrm{TM}}^{H_{\perp}}\left(k_{\perp}\right) \\
c_{\mathrm{TE}}^{H_{\perp}}\left(k_{\perp}\right)
\end{array}\right] d q=\beta\left[\begin{array}{c}
c_{\mathrm{TM}}^{E_{\perp}}\left(k^{\prime}{ }_{\perp}\right) \\
c_{\mathrm{TE}}^{E_{\perp}}\left(k_{\perp}^{\prime}\right)
\end{array}\right]
$$

where

$$
\begin{aligned}
& \overline{\bar{O}}_{e h}\left(k_{\perp}^{\prime}, k_{\perp}\right)=\left[\begin{array}{ll}
O_{e h}^{\mathrm{TMTM}}\left(k^{\prime}{ }_{\perp}, k_{\perp}\right) & O_{e h}^{\mathrm{TMTE}}\left(k^{\prime}{ }_{\perp}, k_{\perp}\right) \\
O_{e h}^{\mathrm{TETM}}\left(k^{\prime}{ }_{\perp}, k_{\perp}\right) & O_{e h}^{\mathrm{TETE}}\left(k^{\prime}, k_{\perp}\right)
\end{array}\right] \\
& =\int\left[\begin{array}{ll}
f_{u, \mathrm{TM}}\left(k^{\prime}\right) & f_{v, \mathrm{TM}}\left(k^{\prime}{ }_{\perp}\right) \\
g_{u, \mathrm{TE}}\left(k^{\prime}\right) & g_{v, \mathrm{TE}}\left(k^{\prime}{ }_{\perp}\right)
\end{array}\right] \hat{O}_{e h}\left[\begin{array}{ll}
g_{u, \mathrm{TM}}\left(k_{\perp}\right) & f_{u, \mathrm{TE}}\left(k_{\perp}\right) \\
g_{v, \mathrm{TM}}\left(k_{\perp}\right) & f_{v, \mathrm{TE}}\left(k_{\perp}\right)
\end{array}\right] d \mathbf{r}_{\perp}
\end{aligned}
$$

As example, the TMTM element is given by

$$
O_{e h}^{\mathrm{TMTM}}\left(k_{\perp}^{\prime}, k_{\perp}\right)=\left[\begin{array}{ll}
f_{u, \mathrm{TM}}\left(k^{\prime}{ }_{\perp}\right) & f_{v, \mathrm{TM}}\left(k^{\prime}{ }_{\perp}\right)
\end{array}\right] \hat{O}_{e h}\left[\begin{array}{l}
g_{u, \mathrm{TM}}\left(k_{\perp}\right) \\
g_{v, \mathrm{TM}}\left(k_{\perp}\right)
\end{array}\right]=\left\langle\mathbf{f}_{\mathrm{TM}}\left(k_{\perp}^{\prime}\right)\left|\hat{O}_{e h}\right| \mathbf{g}_{\mathrm{TM}}\left(k_{\perp}\right)\right\rangle .
$$




\subsection{Evaluation of operator matrix elements}

The derivations of the subelements of the $\overline{\bar{O}}_{e h}\left(k^{\prime}{ }_{\perp}, k_{\perp}\right)$ matrix are very similar to those for the closed geometry. The TMTM element is

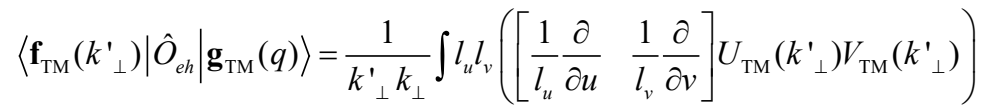

$$
\begin{aligned}
& {\left[\begin{array}{cc}
-\frac{1}{l_{u}} \frac{\partial}{\partial u} \frac{1}{k_{0}^{2} \varepsilon_{r}} \frac{1}{l_{u} l_{v}} \frac{\partial}{\partial v} l_{u} & 1+\frac{1}{l_{u}} \frac{\partial}{\partial u} \frac{1}{k_{0}^{2} \varepsilon_{r}} \frac{1}{l_{u} l_{v}} \frac{\partial}{\partial u} l_{v} \\
-1-\frac{1}{l_{v}} \frac{\partial}{\partial v} \frac{1}{k_{0}^{2} \varepsilon_{r}} \frac{1}{l_{u} l_{v}} \frac{\partial}{\partial v} l_{u} & \frac{1}{l_{v}} \frac{\partial}{\partial v} \frac{1}{k_{0}^{2} \varepsilon_{r}} \frac{1}{l_{u} l_{v}} \frac{\partial}{\partial u} l_{v}
\end{array}\right]\left[\begin{array}{c}
-\frac{1}{l_{v}} \frac{\partial}{\partial v} \\
\frac{1}{l_{u}} \frac{\partial}{\partial u}
\end{array}\right] U_{\mathrm{TM}}\left(k_{\perp}\right) V_{\mathrm{TM}}\left(k_{\perp}\right) d u d v} \\
& =\frac{1}{k_{\perp}^{\prime} k_{\perp}} \int l_{u} l_{v}\left(\left[\frac{1}{l_{u}} \frac{\partial}{\partial u} \frac{1}{l_{v}} \frac{\partial}{\partial v}\right] U_{\mathrm{TM}}\left(k_{\perp}^{\prime}\right) V_{\mathrm{TM}}\left(k_{\perp}^{\prime}\right)\right)\left[\begin{array}{l}
\frac{1}{l} \frac{\partial}{\partial u} \frac{1}{k_{0}^{2} \varepsilon_{r}} \Delta+\frac{1}{l_{u}} \frac{\partial}{\partial u} \\
\frac{1}{l_{v}} \frac{\partial}{\partial v}+\frac{1}{l_{v}} \frac{\partial}{\partial v} \frac{1}{k_{0}^{2} \varepsilon_{r}} \Delta
\end{array}\right] U_{\mathrm{TM}}\left(k_{\perp}\right) V_{\mathrm{TM}}\left(k_{\perp}\right) d u d v \\
& =\frac{1}{k_{\perp}^{\prime} k_{\perp}} \int\left(\begin{array}{l}
\frac{l_{v}}{l_{u}}\left(\frac{\partial}{\partial u} U_{\mathrm{TM}}\left(k_{\perp}^{\prime}\right) V_{\mathrm{TM}}\left(k_{\perp}^{\prime}\right)\right)\left(\frac{\partial}{\partial u}+\frac{\partial}{\partial u} \frac{1}{k_{0}^{2} \varepsilon_{r}} \Delta\right) U_{\mathrm{TM}}\left(k_{\perp}\right) V_{\mathrm{TM}}\left(k_{\perp}\right) \\
+\frac{l_{u}}{l_{v}}\left(\frac{\partial}{\partial v} U_{\mathrm{TM}}\left(k_{\perp}^{\prime}\right) V_{\mathrm{TM}}\left(k_{\perp}^{\prime}\right)\right)\left(\frac{\partial}{\partial v}+\frac{\partial}{\partial v} \frac{1}{k_{0}^{2} \varepsilon_{r}} \Delta\right) U_{\mathrm{TM}}\left(k_{\perp}\right) V_{\mathrm{TM}}\left(k_{\perp}\right)
\end{array}\right) d u d v \\
& =-\frac{1}{k_{\perp}^{\prime} k_{\perp}} \int\left(\left[\frac{\partial}{\partial u} \frac{l_{v}}{l_{u}} \frac{\partial}{\partial u}+\frac{\partial}{\partial v} \frac{l_{u}}{l_{v}} \frac{\partial}{\partial v}\right] U_{\mathrm{TM}}\left(k_{\perp}^{\prime}\right) V_{\mathrm{TM}}\left(k_{\perp}^{\prime}\right)\right)\left(1+\frac{1}{k_{0}^{2} \varepsilon_{r}} \Delta\right) U_{\mathrm{TM}}\left(k_{\perp}\right) V_{\mathrm{TM}}\left(k_{\perp}\right) d u d v \\
& =-\frac{1}{k_{\perp}^{\prime} k_{\perp}} \int l_{u} l_{v}\left(\Delta U_{\mathrm{TM}}\left(k_{\perp}^{\prime}\right) V_{\mathrm{TM}}\left(k_{\perp}^{\prime}\right)\right)\left(1+\frac{1}{k_{0}^{2} \varepsilon_{r}} \Delta\right) U_{\mathrm{TM}}\left(k_{\perp}\right) V_{\mathrm{TM}}\left(k_{\perp}\right) d u d v \\
& =-k_{\perp}^{\prime} k_{\perp} \int l_{u} l_{v} U_{\mathrm{TM}}\left(k_{\perp}^{\prime}\right) V_{\mathrm{TM}}\left(k_{\perp}^{\prime}\right) \frac{1}{k_{0}^{2} \varepsilon_{r}} U_{\mathrm{TM}}\left(k_{\perp}\right) V_{\mathrm{TM}}\left(k_{\perp}\right) d u d v+\delta\left(k_{\perp}^{\prime}-k_{\perp}\right)
\end{aligned}
$$

We now use that $\eta_{r}(u)=1 / \varepsilon_{r}(u)=\eta_{r, \mathrm{~B}}+\Delta \eta_{r}(u)$, where $\Delta \eta_{r}(u)=0$ for $u>u_{\mathrm{B}}$, to write

$$
\begin{aligned}
& \left\langle\mathbf{f}_{\mathrm{TM}}\left(k^{\prime}{ }_{\perp}\right)\left|\hat{O}_{e h}\right| \mathbf{g}_{\mathrm{TM}}\left(k_{\perp}\right)\right\rangle \\
& =-k^{\prime}{ }_{\perp} k_{\perp} \int_{u=0, v=0}^{u=u_{\mathrm{B}}, v=2 \pi} l_{u} l_{v} U_{\mathrm{TM}}\left(k^{\prime}{ }_{\perp}\right) V_{\mathrm{TM}}\left(k^{\prime}{ }_{\perp}\right) \frac{\Delta \eta_{r}(u)}{k_{0}^{2}} U_{\mathrm{TM}}\left(k_{\perp}\right) V_{\mathrm{TM}}\left(k_{\perp}\right) d u d v+\left(1-\frac{\eta_{r, \mathrm{~B}} k_{\perp}^{2}}{k_{0}^{2}}\right) \delta\left(k^{\prime}{ }_{\perp}-k_{\perp}\right)
\end{aligned}
$$

The TMTM contribution to the LHS of (153) then becomes

$$
\begin{aligned}
& \int\left\langle\mathbf{f}_{\mathrm{TM}}\left(k^{\prime}{ }_{\perp}\right)\left|\hat{O}_{e h}\right| \mathbf{g}_{\mathrm{TM}}\left(k_{\perp}\right)\right\rangle c_{\mathrm{TM}}^{H_{\perp}}\left(k_{\perp}\right) d k_{\perp} \\
& =-k^{\prime}{ }_{\perp} k_{\perp} \int_{u=0, v=0}^{u, u_{\mathrm{B}}, 2=2 \pi}\left(l_{u} l_{v} U_{\mathrm{TM}}\left(k^{\prime}{ }_{\perp}\right) V_{\mathrm{TM}}\left(k^{\prime}{ }_{\perp}\right) \frac{\Delta \eta_{r}(u)}{k_{0}^{2}} U_{\mathrm{TM}}\left(k_{\perp}\right) V_{\mathrm{TM}}\left(k_{\perp}\right) d u d v+\left(\frac{\eta_{r, \mathrm{~B}} k_{\perp}^{2}}{k_{0}^{2}}-1\right) \delta\left(k^{\prime}{ }_{\perp}-k_{\perp}\right)\right) c_{\mathrm{TM}}^{H_{\perp}}\left(k_{\perp}\right) d k_{\perp}
\end{aligned}
$$

Introducing the Riemann sum and the replacements and shorthand notations

$$
\begin{gathered}
k_{\perp} \rightarrow k_{\perp, q} \\
k_{\perp}^{\prime} \rightarrow k_{\perp, q^{\prime}} \\
U_{\mathrm{TX}}\left(k_{\perp}\right) \rightarrow U_{\mathrm{TX}}\left(k_{\perp, q}\right)=U_{q, \mathrm{TX}} \\
V_{\mathrm{TX}}\left(k_{\perp}\right) \rightarrow V_{\mathrm{TX}}\left(k_{\perp, q}\right)=V_{q, \mathrm{TX}},
\end{gathered}
$$

leads to 


$$
\begin{aligned}
& \sum_{q}\left\langle\mathbf{f}_{\mathrm{TM}}\left(k_{\perp, q^{\prime}}\right)\left|\hat{O}_{e h}\right| \mathbf{g}_{\mathrm{TM}}\left(k_{\perp, q}\right)\right\rangle c_{\mathrm{TM}}^{H_{\perp}}\left(k_{\perp, q}\right) \Delta k_{\perp, q} \\
& \rightarrow-\sum_{q}\left(k_{\perp, q^{\prime}} k_{\perp, q} \int_{u=0, v=0}^{u=u_{\mathrm{B}}, v=2 \pi} l_{u} l_{v} U_{q^{\prime}, \mathrm{TM}} V_{q^{\prime}, \mathrm{TM}} \frac{\Delta \eta_{r}(u)}{k_{0}^{2}} U_{q, \mathrm{TM}} V_{q, \mathrm{TM}} d u d v \Delta k_{\perp, q}+\delta_{q^{\prime} q}\left(\frac{\eta_{r, \mathrm{~B}} k_{\perp, q}^{2}}{k_{0}^{2}}-1\right)\right) c_{\mathrm{TM}, q}^{H_{\perp}} .
\end{aligned}
$$

The matrix form of Eq. (76) can thus be written

$$
\overline{\bar{O}}_{e h}\left[\begin{array}{l}
\bar{c}_{\mathrm{TM}} \\
\overline{\mathrm{T}}_{\perp} \\
\bar{c}_{\mathrm{TE}}
\end{array}\right]=\left[\begin{array}{ll}
\overline{\bar{O}}_{e h}^{\mathrm{TMTM}} & \overline{\bar{O}}_{e h}^{\mathrm{TMTE}} \\
\overline{\bar{O}}_{e h}^{\mathrm{TETM}} & \overline{\bar{O}}_{e h}^{\mathrm{TETE}}
\end{array}\right]\left[\begin{array}{l}
-H_{\perp} \\
\bar{c}_{\mathrm{TM}} \\
\bar{c}_{\perp} \\
\bar{c}_{\mathrm{TE}}
\end{array}\right]=\beta\left[\begin{array}{c}
-E_{\perp} \\
c_{\mathrm{TM}} \\
\bar{c}_{\perp} \\
\bar{c}_{\mathrm{TE}}
\end{array}\right]
$$

where the TMTM matrix elements are of the form

$$
O_{e h, q^{\prime}, q}^{\mathrm{TMTM}}=-k_{\perp, q} k_{\perp, q} \int_{u=0, v=0}^{u=u_{\mathrm{B}}, v=2 \pi} l_{u} l_{v} U_{q^{\prime}, \mathrm{TM}} V_{q^{\prime}, \mathrm{TM}} \frac{\Delta \eta_{r}(u)}{k_{0}^{2}} U_{q, \mathrm{TM}} V_{q, \mathrm{TM}} d u d v \Delta k_{\perp, q}+\delta_{q^{\prime} q}\left(1-\frac{\eta_{r, \mathrm{~B}} k_{\perp, q}^{2}}{k_{0}^{2}}\right) .
$$

The remaining elements for the $\overline{\bar{O}}_{e h}$ operator matrix are computed using similar principles, and we simply state the results:

$$
\begin{gathered}
O_{e h, q^{\prime} q}^{\mathrm{TETE}}=\delta_{q^{\prime} q} \\
O_{e h, q^{\prime} q}^{\mathrm{TMTE}}=O_{e h, q^{\prime} q}^{\mathrm{TETM}}=0
\end{gathered}
$$

Using a similar procedure, the matrix form of eqs. (77) and (100) become

$$
\begin{gathered}
\overline{\bar{O}}_{h e}=\left[\begin{array}{ll}
\overline{\bar{O}}_{h e}^{\mathrm{TMTM}} & \overline{\bar{O}}_{h e}^{\mathrm{TMTE}} \\
\overline{\bar{O}}_{h e}^{\mathrm{TETM}} & \overline{\bar{O}}_{h e}^{\mathrm{TETE}}
\end{array}\right]\left[\begin{array}{l}
-E_{\perp} \\
c_{\mathrm{TM}} \\
\bar{c}_{\perp}
\end{array}\right]=\beta\left[\begin{array}{l}
\bar{c}_{H_{\perp}} \\
\bar{c}_{H_{\perp}} \\
\bar{c}_{\mathrm{TE}}
\end{array}\right], \\
{\left[\begin{array}{l}
\bar{c}_{\mathrm{TM}} \\
\bar{c}_{\perp} \\
\bar{c}_{\mathrm{TE}}
\end{array}\right]=\overline{\bar{O}}_{d e}\left[\begin{array}{l}
\bar{c}_{\perp} \\
\overline{\mathrm{TM}}_{\mathrm{TM}} \\
\bar{c}_{\perp}
\end{array}\right]=\left[\begin{array}{ll}
\overline{\bar{O}}_{d e}^{\mathrm{TMTM}} & \overline{\bar{O}}_{d e}^{\mathrm{TMTE}} \\
\overline{\bar{O}}_{d e}^{\mathrm{TETM}} & \overline{\bar{O}}_{d e}^{\mathrm{TETE}}
\end{array}\right]\left[\begin{array}{l}
\bar{c}_{\perp} \\
\overline{\mathrm{TM}}_{E_{\perp}} \\
\bar{c}_{\mathrm{TE}}
\end{array}\right],}
\end{gathered}
$$

with matrix elements given by

$$
\begin{gathered}
O_{h, q^{\prime} q}^{\mathrm{TMTM}}=\frac{k_{\perp, q}}{k_{\perp, q^{\prime}}} \int_{u=0, v=0}^{u=u_{\mathrm{B}}, v=2 \pi} l_{u} l_{v} U_{q^{\prime}, \mathrm{TM}} V_{q^{\prime}, \mathrm{TM}} k_{0}^{2} \Delta \varepsilon_{r}(u) U_{q, \mathrm{TM}} V_{q, \mathrm{TM}} d u d v \Delta k_{\perp, q}+\delta_{q^{\prime}} k_{0}^{2} \varepsilon_{r, \mathrm{~B}} \\
-\frac{1}{k_{\perp, q^{\prime}} k_{\perp, q}} \int_{u=0, v=0}^{u=u_{\mathrm{B}}, v=2 \pi} U_{q^{\prime}, \mathrm{TM}} V_{q^{\prime}, \mathrm{TM}} k_{0}^{2}\left(\frac{\partial}{\partial u} \varepsilon_{r}\right) \frac{l_{v}}{l_{u}}\left(\frac{\partial}{\partial u} U_{q, \mathrm{TM}}\right) V_{q, \mathrm{TM}} d u d v \Delta k_{\perp, q} \\
O_{h e, q^{\prime} q}^{\mathrm{TETE}}=\frac{k_{\perp, q}}{k_{\perp, q^{\prime}}} \int_{u=0, v=0}^{u=u_{\mathrm{B}}, v=2 \pi} l_{u} l_{v} U_{q^{\prime}, \mathrm{TE}} V_{q^{\prime}, \mathrm{TE}} k_{0}^{2} \Delta \varepsilon_{r}(u) U_{q, \mathrm{TE}} V_{q, \mathrm{TE}} d u d v \Delta k_{\perp, q}+\delta_{q^{\prime} q}\left(k_{0}^{2} \varepsilon_{r, \mathrm{~B}}-k_{\perp, q}^{2}\right) \\
-\frac{1}{k_{\perp, q^{\prime}} k_{\perp, q}} \int_{u=0, v=0}^{u=u_{b}, v=2 \pi} U_{q^{\prime}, \mathrm{TE}} V_{q^{\prime}, \mathrm{TE}} k_{0}^{2}\left(\frac{\partial}{\partial u} \varepsilon_{r}\right) \frac{l_{v}}{l_{u}}\left(\frac{\partial}{\partial u} U_{q, \mathrm{TE}}\right) V_{q, \mathrm{TE}} d u d v \Delta k_{\perp, q} \\
O_{h e, q^{\prime} q}^{\mathrm{TMTE}}=-\frac{1}{k_{\perp, q^{\prime}} k_{\perp, q}} \int_{u=u_{\mathrm{B}}, v=2 \pi}^{u=0, v=0} U_{q^{\prime}, \mathrm{TM}} V_{q^{\prime}, \mathrm{TM}} k_{0}^{2}\left(\frac{\partial}{\partial u} \varepsilon_{r}\right) U_{q, \mathrm{TE}}\left(q_{m}\right)\left(\frac{\partial}{\partial v} V_{q, \mathrm{TE}}\right) d u d v \Delta k_{\perp, q} \\
O_{h e, q^{\prime} q}^{\mathrm{TETM}}=\frac{1}{k_{\perp, q} k_{\perp, q}} \int_{u=0, v=0}^{u=u_{\mathrm{B}}, v=2 \pi} U_{q^{\prime}, \mathrm{TE}} V_{q^{\prime}, \mathrm{TE}} k_{0}^{2}\left(\frac{\partial}{\partial u} \varepsilon_{r}\right) U_{q, \mathrm{TM}}\left(\frac{\partial}{\partial v} V_{q, \mathrm{TM}}\right) d u d v \Delta k_{\perp, q}
\end{gathered}
$$




$$
\begin{aligned}
& O_{d e, q^{\prime} q}^{\mathrm{TMTM}}=\frac{k_{\perp, q}}{k_{\perp, q^{\prime}}} \int_{u=0, v=0}^{u=u_{\mathrm{B}}, v=2 \pi} l_{u} l_{v} U_{q^{\prime}, \mathrm{TM}} V_{q^{\prime}, \mathrm{TM}} \Delta \varepsilon_{r}(u) U_{q, \mathrm{TM}} V_{q, \mathrm{TM}} d u d v \Delta k_{\perp, q}+\delta_{q^{\prime} q} \varepsilon_{r, \mathrm{~B}} \\
& -\frac{1}{k_{\perp, q^{\prime}} k_{\perp, q}} \int_{u=u_{b}, v=2 \pi}^{u=0, v=0} U_{q^{\prime}, \mathrm{TM}} V_{q^{\prime}, \mathrm{TM}}\left(\frac{\partial}{\partial u} \varepsilon_{r}\right) \frac{l_{v}}{l_{u}}\left(\frac{\partial}{\partial u} U_{q, \mathrm{TM}}\right) V_{q, \mathrm{TM}} d u d v \Delta k_{\perp, q} \\
& O_{d e, q^{\prime} q}^{\mathrm{TETE}}=\frac{k_{\perp, q}}{k_{\perp, q^{\prime}}} \int_{u=0, v=0}^{u=u_{\mathrm{B}}, v=2 \pi} l_{u} l_{v} U_{q^{\prime}, \mathrm{TE}} V_{q^{\prime}, \mathrm{TE}} \Delta \varepsilon_{r}(u) U_{q, \mathrm{TE}} V_{q, \mathrm{TE}} d u d v \Delta k_{\perp, q}+\varepsilon_{r, \mathrm{~B}} \delta_{q^{\prime} q} \\
& -\frac{1}{k_{\perp, q^{\prime}} k_{\perp, q}} \int_{u=0, v=0}^{u=u_{\mathrm{B}}, v=2 \pi} U_{q^{\prime}, \mathrm{TE}} V_{q^{\prime}, \mathrm{TE}}\left(\frac{\partial}{\partial u} \varepsilon_{r}\right) \frac{l_{v}}{l_{u}}\left(\frac{\partial}{\partial u} U_{q, \mathrm{TE}}\right) V_{q, \mathrm{TE}} d u d v \Delta k_{\perp, q} \\
& O_{d e, q^{\prime} q}^{\mathrm{TMTE}}=-\frac{1}{k_{\perp, q^{\prime}} k_{\perp, q}} \int_{u=u_{\mathrm{B}}, v=2 \pi}^{u=0, v=0} U_{q^{\prime}, \mathrm{TM}} V_{q^{\prime}, \mathrm{TM}}\left(\frac{\partial}{\partial u} \varepsilon_{r}\right) U_{q, \mathrm{TE}}\left(\frac{\partial}{\partial v} V_{q, \mathrm{TE}}\right) d u d v \Delta k_{\perp, q} \\
& O_{d e, q^{\prime} q}^{\mathrm{TETM}}=\frac{1}{k_{\perp, q^{\prime}} k_{\perp, q}} \int_{u=0, v=0}^{u=2 \pi} U_{q^{\prime}, \mathrm{TE}} V_{q^{\prime}, \mathrm{TE}}\left(\frac{\partial}{\partial u} \varepsilon_{r}\right) U_{q, \mathrm{TM}}\left(\frac{\partial}{\partial v} V_{q, \mathrm{TM}}\right) d u d v \Delta k_{\perp, q} .
\end{aligned}
$$

The $z$ components of the fields can be computed using (73)-(74), which can be written in matrix form as

$$
\begin{aligned}
& \bar{c}^{E_{z}}=\overline{\bar{O}}_{E_{z} H} \bar{c}_{\mathrm{TM}} \\
& \bar{c}^{H_{z}}=\overline{\bar{O}}_{H_{z} E} \bar{c}_{\mathrm{TE}},
\end{aligned}
$$

where the matrix elements are given by

$$
\begin{gathered}
O_{E_{z} H, q^{\prime} q}=-i k_{\perp, q} \int_{u=0, v=0}^{u=u_{\mathrm{B}}, v=2 \pi} U_{q^{\prime}, \mathrm{TM}} V_{q^{\prime}, \mathrm{TM}} \frac{\Delta \eta_{r}}{k_{0}^{2}} U_{q, \mathrm{TM}} V_{q, \mathrm{TM}} l_{u} l_{v} d u d v \Delta k_{\perp, q}-\frac{i k_{\perp, q} \eta_{r, \mathrm{~B}}}{k_{0}^{2}} \delta_{q^{\prime} q} \\
O_{H_{z} E, m^{\prime} m}=-i k_{\perp, m} \delta_{m^{\prime} m} .
\end{gathered}
$$

\subsection{Orthonormalization of eigenmodes}

Again, eigenmodes with different propagation constants are orthogonal to each other with respect to the product

$$
\int\left(\mathbf{e}_{j} \times \mathbf{h}_{j^{\prime}}\right) \cdot \mathbf{z} d \mathbf{r}_{\perp}=0 \quad\left(j \neq j^{\prime}\right),
$$

where $j$ and $j$ ' are universal indices referring either to modes from the discretized set of guided modes or from the continuous sets of TE or TM radiation modes.

For the guided mode $\left(\mathbf{e}_{\perp, j}^{G}, \mathbf{h}_{\perp, j}^{G}\right)$ of discrete index $j$, the normalization constant is computed as

$$
\int\left(\mathbf{e}_{\perp, j}^{G} \times \mathbf{h}_{\perp, j}^{G}\right) \cdot \mathbf{z} d \mathbf{r}_{\perp}=N_{j}^{G} .
$$

We then insert the field expansions (139)-(140) for the $\mathrm{j}$ 'th mode into (183) using the relations

$$
\begin{gathered}
\int\left(\mathbf{f}_{\mathrm{TM}}\left(k_{\perp}^{\prime}\right) \times \mathbf{g}_{\mathrm{TM}}\left(k_{\perp}\right)\right) \cdot \mathbf{z} d \mathbf{r}_{\perp}=\left\langle\mathbf{f}_{\mathrm{TM}}\left(k_{\perp}^{\prime}\right) \mid \mathbf{f}_{\mathrm{TM}}\left(k_{\perp}\right)\right\rangle=\delta\left(k_{\perp}^{\prime}-k_{\perp}\right) \\
\int\left(\mathbf{g}_{\mathrm{TE}}\left(k_{\perp}^{\prime}\right) \times \mathbf{f}_{\mathrm{TE}}\left(k_{\perp}\right)\right) \cdot \mathbf{z} d \mathbf{r}_{\perp}=\left\langle\mathbf{g}_{\mathrm{TE}}\left(k_{\perp}^{\prime}\right) \mid \mathbf{g}_{\mathrm{TE}}\left(k_{\perp}\right)\right\rangle=\delta\left(k_{\perp}^{\prime}-k_{\perp}\right) \\
\int\left(\mathbf{f}_{\mathrm{TM}}\left(k_{\perp}^{\prime}\right) \times \mathbf{f}_{\mathrm{TE}}\left(k_{\perp}\right)\right) \cdot \mathbf{z} d \mathbf{r}_{\perp}=\left\langle\mathbf{f}_{\mathrm{TM}}\left(k_{\perp}^{\prime}\right) \mid \mathbf{g}_{\mathrm{TE}}\left(k_{\perp}\right)\right\rangle=0 \\
\int\left(\mathbf{g}_{\mathrm{TE}}\left(k_{\perp}^{\prime}\right) \times \mathbf{g}_{\mathrm{TM}}\left(k_{\perp}\right)\right) \cdot \mathbf{z} d \mathbf{r}_{\perp}=\left\langle\mathbf{g}_{\mathrm{TE}}\left(k_{\perp}^{\prime}\right) \mid \mathbf{f}_{\mathrm{TM}}\left(k_{\perp}\right)\right\rangle=0,
\end{gathered}
$$

which leads to 


$$
\begin{aligned}
& \int\left(\mathbf{e}_{\perp, j}^{G} \times \mathbf{h}_{\perp, j}^{G}\right) \cdot \mathbf{z} d \mathbf{r}_{\perp}=\int\left(c_{\mathrm{TM}, j}^{E_{\perp}}\left(k_{\perp}\right) c_{\mathrm{TM}, j}^{H_{\perp}}\left(k_{\perp}\right)+c_{\mathrm{TE}, j}^{E_{\perp}}\left(k_{\perp}\right) c_{\mathrm{TE}, j}^{H_{\perp}}\left(k_{\perp}\right)\right) d k_{\perp} \\
& \rightarrow \sum_{q}\left(c_{\mathrm{TM}, q j}^{E_{\perp}} c_{\mathrm{TM}, q j}^{H_{\perp}} \Delta k_{\perp, q}+c_{\mathrm{TE}, q j}^{E_{\perp}} c_{\mathrm{TE}, q j}^{H_{\perp}} \Delta k_{\perp, q}\right)=N_{j}^{G}
\end{aligned}
$$

and as before the normalization is obtained by performing the replacement (133).

In the case of radiation modes, $j$ is now a continuous index including the polarization indices TM and TE and Eq. (182) becomes

$$
\int\left(\mathbf{e}_{\perp}^{R}\left(j^{\prime}\right) \times \mathbf{h}_{\perp}^{R}(j)\right) \cdot \mathbf{z} d \mathbf{r}_{\perp}=N_{j}^{R} \delta\left(j^{\prime}-j\right),
$$

where the continuous $j$ index has been placed in the parenthesis for convenience in the following. Inserting the expansions Eqs. (139)-(140) for the eigenmode fields and exploiting the orthogonality relations Eqs. (137)-(138), we obtain

$$
\int\left(c_{\mathrm{TM}}^{E_{\perp}}\left(j^{\prime}, k_{\perp}\right) c_{\mathrm{TM}}^{H_{\perp}}\left(j, k_{\perp}\right)+c_{\mathrm{TE}}^{E_{\perp}}\left(j^{\prime}, k_{\perp}\right) c_{\mathrm{TE}}^{H_{\perp}}\left(j, k_{\perp}\right)\right) d k_{\perp}=N_{j}^{R} \delta\left(j^{\prime}-j\right) .
$$

We now introduce the Riemann sum for the integrals over $k_{\perp}$ and $j$

$$
\begin{gathered}
\int f\left(k_{\perp}\right) d k_{\perp} \rightarrow \sum_{q} f\left(k_{\perp}\right) \Delta k_{\perp, q} \\
\int f(j) d j \rightarrow \sum_{p} f\left(j_{p}\right) \Delta j_{p}
\end{gathered}
$$

and we define

$$
N_{j_{p}}^{R, d} \equiv \sum_{q}\left(c_{\mathrm{TM}, q}^{E_{\perp}}\left(j_{p}\right) c_{\mathrm{TM}, q}^{H_{\perp}}\left(j_{p}\right)+c_{\mathrm{TE}, q}^{E_{\perp}}\left(j_{p}\right) c_{\mathrm{TE}, q}^{H_{\perp}}\left(j_{p}\right)\right) \Delta k_{\perp, q}
$$

Performing the integration over $j^{\prime}$ in Eq. (190) using the Riemann sums (191) and (192), we obtain

$$
\sum_{q, p^{\prime}}\left(c_{\mathrm{TM}, q}^{E_{\perp}}\left(j_{p^{\prime}}\right) c_{\mathrm{TM}, q}^{H_{\perp}}\left(j_{p}\right)+c_{\mathrm{TE}, q}^{E_{\perp}}\left(j_{p^{\prime}}\right) c_{\mathrm{TE}, q}^{H_{\perp}}\left(j_{p}\right)\right) \Delta k_{\perp, q} \Delta j_{p^{\prime}}=\sum_{p^{\prime}} \delta_{p^{\prime} p} N_{j_{p}}^{R, d} \Delta j_{p^{\prime}}=N_{j_{p}}^{R, d} \Delta j_{p}=N_{j_{p}}^{R}=N_{j}^{R},
$$

where we have exploited mode orthogonality in the second step.

We now choose a normalization of the radiation modes by performing the replacement (133) with $N_{j_{p}}^{R, d}$. We observe that $N_{j_{p}}^{R, d}$ is defined in the same way as $N_{j}^{G}$, and we can use identical code to treat both the guided modes and the radiation modes. With this choice we obtain

$$
N_{j}^{R}=N_{j_{p}}^{R}=\Delta j_{p}
$$

which we will use in this form in the next section.

\subsection{Current sources in a uniform geometry}

We now consider the optical field $\left(\mathbf{E}_{1}, \mathbf{H}_{1}\right)$ generated by a current source of the form

$$
\mathbf{J}=\mathbf{J}_{1}=\mathbf{J}(x, y) \delta\left(z-z_{\mathrm{J}}\right) .
$$

The generated fields can be written [1] as

$$
\begin{aligned}
& \mathbf{E}_{1}(\mathbf{r})=\sum_{j} a_{j}^{\mathrm{J}, G} \mathbf{e}_{j}^{G,+}\left(\mathbf{r}_{\perp}\right) e^{i \beta_{j}^{G}\left(z-z_{\mathrm{J}}\right)}+\int a_{j}^{\mathrm{J}, R} \mathbf{e}_{j}^{R,+}\left(\mathbf{r}_{\perp}\right) e^{i \beta_{j}^{R}\left(z-z_{\mathrm{J}}\right)} d j \quad\left(z \geq z_{\mathrm{J}}\right) \\
& \mathbf{H}_{1}(\mathbf{r})=\sum_{j} a_{j}^{\mathrm{J}, G} \mathbf{h}_{j}^{G,+}\left(\mathbf{r}_{\perp}\right) e^{i \beta_{j}^{G}\left(z-z_{\mathrm{J}}\right)}+\int a_{j}^{\mathrm{J}, R} \mathbf{h}_{j}^{R,+}\left(\mathbf{r}_{\perp}\right) e^{i \beta_{j}^{R}\left(z-z_{\mathrm{J}}\right)} d j \quad\left(z \geq z_{\mathrm{J}}\right)
\end{aligned}
$$




$$
\begin{aligned}
& \mathbf{E}_{1}(\mathbf{r})=\sum_{j} b_{j}^{\mathrm{J}, G} \mathbf{e}_{j}^{G,-}\left(\mathbf{r}_{\perp}\right) e^{-i \beta_{j}^{G}\left(z-z_{\mathrm{J}}\right)}+\int b_{j}^{\mathrm{J}, R} \mathbf{e}_{j}^{R,-}\left(\mathbf{r}_{\perp}\right) e^{-i \beta_{j}^{R,-}\left(z-z_{\mathrm{J}}\right)} d j \quad\left(z \leq z_{\mathrm{J}}\right) \\
& \mathbf{H}_{1}(\mathbf{r})=\sum_{j} b_{j}^{\mathrm{J}, G} \mathbf{h}_{j}^{G,+}\left(\mathbf{r}_{\perp}\right) e^{-i \beta_{j}^{G}\left(z-z_{\mathrm{J}}\right)}+\int b_{j}^{\mathrm{J}, R} \mathbf{h}_{j}^{R,-}\left(\mathbf{r}_{\perp}\right) e^{-i \beta_{j}^{R}\left(z-z_{\mathrm{J}}\right)} d j \quad\left(z \leq z_{\mathrm{J}}\right),
\end{aligned}
$$

where the polarization indices TM and TE for the radiation modes as before have been absorbed in the continuous index $j$, which now appears as a subindex for compactness. Introducing the Riemann sum (192) for the $j$ integration, we obtain

$$
\begin{aligned}
& \mathbf{E}_{1}(\mathbf{r})=\sum_{j} a_{j}^{\mathrm{J}, G} \mathbf{e}_{j}^{G,+}\left(\mathbf{r}_{\perp}\right) e^{i \beta_{j}^{G}\left(z-z_{\mathrm{J}}\right)}+\sum_{p} a_{j_{p}}^{\mathrm{J}, R} \mathbf{e}_{j_{p}}^{R,+}\left(\mathbf{r}_{\perp}\right) e^{i \beta_{j_{p}}^{R}\left(z-z_{\mathrm{J}}\right)} \Delta j_{p}\left(z \geq z_{\mathrm{J}}\right) \\
& \mathbf{H}_{1}(\mathbf{r})=\sum_{j} a_{j}^{\mathrm{J}, G} \mathbf{h}_{j}^{G,+}\left(\mathbf{r}_{\perp}\right) e^{i \beta_{j}^{G}\left(z-z_{\mathrm{J}}\right)}+\sum_{p} a_{j_{p}}^{\mathrm{J}, R} \mathbf{h}_{j_{p}}^{R,+}\left(\mathbf{r}_{\perp}\right) e^{i \beta_{j_{p}}^{R}\left(z-z_{\mathrm{J}}\right)} \Delta j_{p} \quad\left(z \geq z_{\mathrm{J}}\right) \\
& \mathbf{E}_{1}(\mathbf{r})=\sum_{j} b_{j}^{\mathrm{J}, G} \mathbf{e}_{j}^{G,-}\left(\mathbf{r}_{\perp}\right) e^{-i \beta_{j}^{G}\left(z-z_{\mathrm{J}}\right)}+\sum_{p} b_{j_{p}^{\mathrm{J}}, R}^{\mathbf{e}_{j_{p}}^{R,-}\left(\mathbf{r}_{\perp}\right) e^{-i \beta_{j_{p}}^{R,-}\left(z-z_{\mathrm{J}}\right)} \Delta j_{p}} \quad\left(z \leq z_{\mathrm{J}}\right) \\
& \mathbf{H}_{1}(\mathbf{r})=\sum_{j} b_{j}^{\mathrm{J}, G} \mathbf{h}_{j}^{G,+}\left(\mathbf{r}_{\perp}\right) e^{-i \beta_{j}^{G}\left(z-z_{\mathrm{J}}\right)}+\sum_{p} b_{j_{p}}^{\mathrm{J}, R} \mathbf{h}_{j_{p}}^{R,-}\left(\mathbf{r}_{\perp}\right) e^{-i \beta_{j_{p}}^{R}\left(z-z_{\mathrm{J}}\right)} \Delta j_{p} \quad\left(z \leq z_{\mathrm{J}}\right),
\end{aligned}
$$

which can be written compactly as

$$
\begin{aligned}
& \mathbf{E}_{1}(\mathbf{r})=\sum_{p} a_{p}^{\mathrm{J}, X} \mathbf{e}_{p}^{X,+}\left(\mathbf{r}_{\perp}\right) e^{i \beta_{p}^{X}\left(z-z_{\mathrm{J}}\right)} \quad\left(z \geq z_{\mathrm{J}}\right) \\
& \mathbf{H}_{1}(\mathbf{r})=\sum_{p} a_{p}^{\mathrm{J}, X} \mathbf{h}_{p}^{X,+}\left(\mathbf{r}_{\perp}\right) e^{i \beta_{p}^{X}\left(z-z_{\mathrm{J}}\right)} \quad\left(z \geq z_{\mathrm{J}}\right) \\
& \mathbf{E}_{1}(\mathbf{r})=\sum_{p} b_{p}^{\mathrm{J}, X} \mathbf{e}_{p}^{X,-}\left(\mathbf{r}_{\perp}\right) e^{-i \beta_{p}^{X}\left(z-z_{\mathrm{J}}\right)} \quad\left(z \leq z_{\mathrm{J}}\right) \\
& \mathbf{H}_{1}(\mathbf{r})=\sum_{p} b_{p}^{\mathrm{J}, X} \mathbf{h}_{p}^{X,+}\left(\mathbf{r}_{\perp}\right) e^{-i \beta_{p}^{X}\left(z-z_{\mathrm{J}}\right)} \quad\left(z \leq z_{\mathrm{J}}\right) .
\end{aligned}
$$

Here the sum over $p$ is to be understood as a global sum over all modes, both guided modes and discretized radiation TE and TM modes, obtained by solving the discretized eigenvalue problem in MATLAB. The expansion coefficients are then given by

$$
\begin{aligned}
& a_{p}^{\mathrm{J}, X}=\left\{\begin{array}{cc}
a_{p}^{\mathrm{J}, G} & (X=G) \\
a_{p}^{\mathrm{J}, R} \Delta j_{p} & (X=R)
\end{array}\right. \\
& b_{p}^{\mathrm{J}, X}=\left\{\begin{array}{cc}
b_{p}^{\mathrm{J}, G} & (X=G) \\
b_{p}^{\mathrm{J}, R} \Delta j_{p} & (X=R)
\end{array}\right.
\end{aligned}
$$

The expansion coefficients for the guided modes $a_{p}^{\mathrm{J}, G}$ and $b_{p}^{\mathrm{J}, G}$ are calculated using the procedure discussed in Ref. [1] as

$$
\begin{aligned}
& a_{p}^{\mathrm{J}, \mathrm{G}}=-\frac{1}{2} \int_{S_{\mathrm{J}}}\left(\mathbf{J} \cdot \mathbf{e}_{p}^{X,+}\right) d S_{\mathrm{J}} \\
& b_{p}^{\mathrm{J}, \mathrm{G}}=-\frac{1}{2} \int_{S_{\mathrm{J}}}\left(\mathbf{J} \cdot \mathbf{e}_{p}^{X,-}\right) d S_{\mathrm{J}} .
\end{aligned}
$$

The expansion functions $a_{p}^{\mathrm{J}, X}=a_{p}^{\mathrm{J}, R} \Delta j_{p}$ and $b_{j_{p}}^{\mathrm{J}, X}=b_{j_{p}}^{\mathrm{J}, R} \Delta j_{p}$ for the radiation modes are computed using a similar procedure again taking advantage of the reciprocity theorem given by

$$
\int_{S}\left(\mathbf{E}_{1} \times \mathbf{H}_{2}-\mathbf{E}_{2} \times \mathbf{H}_{1}\right) \cdot \hat{\mathbf{n}} d S=\int_{V}\left(\mathbf{J}_{1} \cdot \mathbf{E}_{2}-\mathbf{J}_{2} \cdot \mathbf{E}_{1}\right) d V
$$


see Ref. [1] for the details of the notation.

We now consider the test field given by $\mathbf{J}_{2}=0$ and $\left(\mathbf{E}_{2}, \mathbf{H}_{2}\right)=\left(\mathbf{e}_{j_{p^{\prime}}}^{R,+}, \mathbf{h}_{j_{p^{\prime}}}^{R,+}\right)$. The reciprocity theorem for this configuration becomes

$$
\begin{aligned}
& -\int_{S_{1}}\left(\mathbf{E}_{1} \times \mathbf{h}_{j_{p^{\prime}}}^{R,+}-\mathbf{e}_{j_{p^{\prime}}}^{R,+} \times \mathbf{H}_{1}\right) \cdot \hat{\mathbf{z}} e^{i \beta_{p^{\prime}}^{R}\left(z_{1}-z_{\mathrm{J}}\right)} d S_{1} \\
& +\int_{S_{2}}\left(\mathbf{E}_{1} \times \mathbf{h}_{j_{p^{\prime}}}^{R,+}-\mathbf{e}_{j_{p^{\prime}}}^{R,+} \times \mathbf{H}_{1}\right) \cdot \hat{\mathbf{z}} e^{i \beta_{j^{\prime}}^{R}\left(z_{2}-z_{\mathrm{J}}\right)} d S_{2}=\int_{S_{\mathrm{J}}}\left(\mathbf{J} \cdot \mathbf{e}_{j_{p^{\prime}}}^{R,+}\right) d S_{\mathrm{J}},
\end{aligned}
$$

where we will let $z_{1} \rightarrow z_{\mathrm{J}}$ and $z_{2} \rightarrow z_{\mathrm{J}}$. The $z$ components of the fields do not contribute to the integrals and we may replace the eigenmodes by their in-plane components. Insertion of the expansions (197)-(200) leads to

$$
\begin{aligned}
& -\int_{S_{\mathrm{J}}}\left[\sum_{j} b_{j}^{\mathrm{J}, \mathrm{G}}\left(\mathbf{e}_{\perp, j}^{G,-} \times \mathbf{h}_{\perp, j_{p^{\prime}}}^{R,+}-\mathbf{e}_{\perp, j_{p^{\prime}}}^{R,+} \times \mathbf{h}_{\perp, j}^{G,-}\right)+\int b_{j}^{\mathrm{J}, R}\left(\mathbf{e}_{j}^{R,-}\left(\mathbf{r}_{\perp}\right) \times \mathbf{h}_{\perp, j_{p^{\prime}}}^{R,+}-\mathbf{e}_{\perp, j_{p^{\prime}}}^{R,+} \times \mathbf{h}_{\perp, j}^{R,-}\right) d j\right] \cdot \hat{\mathbf{z}} d S_{\mathrm{J}} \\
& +\int_{S_{\mathrm{J}}}\left[\sum_{j} a_{j}^{\mathrm{J}, \mathrm{G}}\left(\mathbf{e}_{\perp, j}^{G,+} \times \mathbf{h}_{\perp, j_{p^{\prime}}}^{R,+}-\mathbf{e}_{\perp, j_{p^{\prime}}}^{R,+} \times \mathbf{h}_{\perp, j}^{G,+}\right)+\int a_{j}^{\mathrm{J}, R}\left(\mathbf{e}_{j}^{R,+}\left(\mathbf{r}_{\perp}\right) \times \mathbf{h}_{\perp, j_{p^{\prime}}}^{R,+}-\mathbf{e}_{\perp, j_{p^{\prime}}}^{R,+} \times \mathbf{h}_{\perp, j}^{R,+}\right) d j\right] \cdot \hat{\mathbf{z}} d S_{\mathrm{J}}, \\
& =\int_{S_{\mathrm{J}}}\left(\mathbf{J} \cdot \mathbf{e}_{j_{p^{\prime}}}^{R,+}\right) d S_{\mathrm{J}}
\end{aligned}
$$

Using the conventions $\mathbf{e}_{\perp, j}^{R,-}=\mathbf{e}_{\perp, j}^{R,+}=\mathbf{e}_{\perp, j}^{R}$ and $\mathbf{h}_{\perp, j}^{R,-}=-\mathbf{h}_{\perp, j}^{R,+}=-\mathbf{h}_{\perp, j}^{R}$, the orthogonality relations Eqs. (182) and (189) and the normalizations Eqs. (189) and (195), we obtain

$$
b_{j_{p^{\prime}}}^{\mathrm{J}, R} N_{j_{p^{\prime}}}^{R}=b_{j_{p^{\prime}}}^{\mathrm{J}, R} \Delta j_{p^{\prime}}=b_{p^{\prime}}^{\mathrm{J}, \mathrm{X}}=-\frac{1}{2} \int_{S_{\mathrm{J}}}\left(\mathbf{J} \cdot \mathbf{e}_{j_{p^{\prime}}}^{R,+}\right) d S_{\mathrm{J}} .
$$

A similar procedure leads to

$$
a_{j_{p^{\prime}}}^{\mathrm{J}, R} N_{j_{p^{\prime}}}^{R}=a_{j_{p^{\prime}}}^{\mathrm{J}, R} \Delta j_{p^{\prime}}=a_{p^{\prime}}^{\mathrm{J}, \mathrm{X}}=-\frac{1}{2} \int_{S_{\mathrm{J}}}\left(\mathbf{J} \cdot \mathbf{e}_{j_{p^{\prime}}}^{R,-}\right) d S_{\mathrm{J}} .
$$

Since the expression for the guided mode coefficients $a_{p}^{\mathrm{J}, X}=a_{p}^{\mathrm{J}, G}$ and $b_{p}^{\mathrm{J}, X}=b_{p}^{\mathrm{J}, G}$ are of the same form, we note that using the normalization discussed in Section 4.2, all expansion coefficients, for both guided and radiation modes, can be determined from

$$
\begin{aligned}
& a_{p}^{\mathrm{J}, \mathrm{X}}=-\frac{1}{2} \int_{S_{\mathrm{J}}}\left(\mathbf{J} \cdot \mathbf{e}_{p}^{X,-}\right) d S_{\mathrm{J}} \\
& b_{p}^{\mathrm{J}, \mathrm{X}}=-\frac{1}{2} \int_{S_{\mathrm{J}}}\left(\mathbf{J} \cdot \mathbf{e}_{p}^{X,+}\right) d S_{\mathrm{J}},
\end{aligned}
$$

where $p$ refers to either a discrete index $j$ for a guided mode or a discrete index $j_{p}$ of the discretized continuous set of radiation mode.

\subsection{Power emitted by a radiating dipole}

Having computed the expansion coefficients for the field generated by a current distribution, we can now compute the power $P$ emitted by the current distribution (196). It is given as ${ }^{1}$

$$
P=-\frac{1}{2} \int \operatorname{Re}\left(\mathbf{J}^{*}\left(\mathbf{r}_{\perp}\right) \cdot \mathbf{E}\left(\mathbf{r}_{\perp}\right)\right) d \mathbf{r}_{\perp},
$$

\footnotetext{
${ }^{1}$ Chap. 8 in L. Novotny and B. Hecht, ”Principles of Nano-Optics" (Cambridge University Press 2012).
} 
where the integration is performed over the plane $z=z \mathrm{~J}$. Inserting the expansion given by (217) and (201) into (220), we obtain

$$
P=\frac{1}{4} \int \operatorname{Re}\left(\sum_{p}\left[\mathbf{J}\left(\mathbf{r}_{\perp}^{\prime}\right) \cdot \mathbf{e}_{p}^{X,-}\left(\mathbf{r}_{\perp}^{\prime}\right)\right] \mathbf{J}^{*}\left(\mathbf{r}_{\perp}\right) \cdot \mathbf{e}_{p}^{X,+}\left(\mathbf{r}_{\perp}\right)\right) d \mathbf{r}_{\perp}^{\prime} d \mathbf{r}_{\perp} .
$$

In the case of a point dipole of the form $\mathbf{J}=\mathbf{J}_{\mathrm{PD}} \delta\left(\mathbf{r}-\mathbf{r}_{\mathrm{PD}}\right)$, the spatial integrations can be carried out and the power will be given by

$$
P=\frac{1}{4} \operatorname{Re}\left(\sum_{p}\left[\mathbf{J}_{\mathrm{PD}}\left(\mathbf{r}_{\mathrm{PD}}\right) \cdot \mathbf{e}_{p}^{X}\left(\mathbf{r}_{\mathrm{PD}}\right)\right] \mathbf{J}_{\mathrm{PD}}^{*}\left(\mathbf{r}_{\mathrm{PD}}\right) \cdot \mathbf{e}_{p}^{X}\left(\mathbf{r}_{\mathrm{PD}}\right)\right),
$$

where the \pm superscripts have been skipped due to the convention $\mathbf{e}_{\perp, p}^{X,-}=\mathbf{e}_{\perp, p}^{X,+}=\mathbf{e}_{\perp, p}^{X}$.

For convergence studies, it is often of interest to evaluate the power as function of $k_{\perp}$ such that

$$
P=\int P\left(k_{\perp}\right) d k_{\perp} \rightarrow \sum_{q} P_{\mathrm{TM}}\left(k_{\perp, q}\right) \Delta k_{\perp, q}+\sum_{q} P_{\mathrm{TE}}\left(k_{\perp, q}\right) \Delta k_{\perp, q},
$$

where

$$
\begin{aligned}
& P_{\mathrm{TM}}\left(k_{\perp, q}\right)=\frac{1}{4} \operatorname{Re}\left(\sum_{p}\left[\mathbf{J}_{\mathrm{PD}}\left(\mathbf{r}_{\mathrm{PD}}\right) \cdot \mathbf{e}_{p}^{X}\left(\mathbf{r}_{\mathrm{PD}}\right)\right] \mathbf{J}_{\mathrm{PD}}^{*}\left(\mathbf{r}_{\mathrm{PD}}\right) \cdot c_{\mathrm{TM}, q p}^{E_{\perp}} \mathbf{f}_{\mathrm{TM}}\left(k_{\perp, q}, \mathbf{r}_{\mathrm{PD}}\right)\right) \\
& P_{\mathrm{TE}}\left(k_{\perp, q}\right)=\frac{1}{4} \operatorname{Re}\left(\sum_{p}\left[\mathbf{J}_{\mathrm{PD}}\left(\mathbf{r}_{\mathrm{PD}}\right) \cdot \mathbf{e}_{p}^{X}\left(\mathbf{r}_{\mathrm{PD}}\right)\right] \mathbf{J}_{\mathrm{PD}}^{*}\left(\mathbf{r}_{\mathrm{PD}}\right) \cdot c_{\mathrm{TE}, q p}^{E_{\perp}} \mathbf{g}_{\mathrm{TE}}\left(k_{\perp, q}, \mathbf{r}_{\mathrm{PD}}\right)\right) .
\end{aligned}
$$

and similarly for TE. A convergence study may then consist of plotting $P_{\mathrm{TM}}\left(k_{\perp, q}\right)$ and $P_{\mathrm{TE}}\left(k_{\perp, q}\right)$ as function of $k_{\perp, q}$ to verify that the $k_{\perp}$ space has been properly resolved in the discretization.

\section{Circular cylindrical coordinates}

For the rotationally symmetric geometry we have $(u, v)=(r, \phi), U(u)=R(r)$ and $V(v)=\Phi(\phi)$ and

$$
\begin{array}{rl}
x=\cos (\phi) r & y=\sin (\phi) r \\
l_{r}=1 & l_{\phi}=r
\end{array}
$$

such that the scalar Eq. (13) for the empty geometry becomes

$$
\left(\frac{1}{r} \frac{\partial}{\partial r} r \frac{\partial}{\partial r}+\frac{1}{r^{2}} \frac{\partial^{2}}{\partial \phi^{2}}\right) R(r) \Phi(\phi)+k_{\perp}^{2} R(r) \Phi(\phi)=0 .
$$

For the angular function $\Phi(\phi)$, we may choose a sine, cosine or an exponential function of the forms

$$
\begin{gathered}
\Phi(\phi)=\cos (m \phi) \\
\Phi(\phi)=\sin (m \phi) \\
\Phi(\phi)=\exp ( \pm i m \phi),
\end{gathered}
$$

such that Eq. (228) becomes

$$
\left(\frac{1}{r} \frac{\partial}{\partial r} r \frac{\partial}{\partial r}-\frac{m^{2}}{r^{2}}+k_{\perp}^{2}\right) R(r)=0 .
$$

The solution to Eq. (232) is the Bessel function $J_{m}\left(k_{\perp} r\right)$ of the first kind of order $m$ fulfilling 


$$
\left(\frac{1}{r} \frac{\partial}{\partial r} r \frac{\partial}{\partial r}-\frac{m^{2}}{r^{2}}+k_{\perp}^{2}\right) J_{m}\left(k_{\perp} r\right)=0 .
$$

We should now choose the basis function for the angular dependence. In this work, we will use cosine and sinus functions instead of exponential functions. Inspection of the operator matrix elements (98)-(99) reveals that the TM (TE) modes with either cosine or sine dependence do not couple with TE (TM) modes with the same dependence. We should thus choose TM and TE basis functions with different angular dependences. We now choose a cosine function for TM modes and a sinus function for TE modes. The opposite choice is also possible, and this will lead to modes with orthogonal polarization.

$$
\begin{aligned}
& V_{\mathrm{TM}}(m, v)=\Phi_{\mathrm{TM}}(m, \phi)=\cos (m \phi) \\
& V_{\mathrm{TE}}(m, v)=\Phi_{\mathrm{TE}}(m, \phi)=\sin (m \phi) .
\end{aligned}
$$

Our basis functions are thus given by

$$
\begin{aligned}
& \Psi_{\mathrm{TM}}\left(k_{\perp}, m, r, \phi\right)=N_{m}\left(k_{\perp}\right)^{-1 / 2} J_{m}\left(k_{\perp} r\right) \cos (m \phi) \\
& \Psi_{\mathrm{TE}}\left(k_{\perp}, m, r, \phi\right)=N_{m}\left(k_{\perp}\right)^{-1 / 2} J_{m}\left(k_{\perp} r\right) \sin (m \phi) .
\end{aligned}
$$

For the open geometry, the normalization constant for TM polarization is found using

$$
\begin{aligned}
& \int_{0}^{\infty} \int_{0}^{2 \pi} J_{m}\left(k_{\perp} r\right) \cos (m \phi) J_{m}\left(k_{\perp}^{\prime} r\right) \cos (m \phi) d \phi r d r \\
& =\int_{0}^{2 \pi} \cos (m \phi) \cos (m \phi) d \phi \int_{0}^{\infty} J_{m}\left(k_{\perp} r\right) J_{m}\left(k_{\perp}^{\prime} r\right) r d r . \\
& =N_{\phi, m} N_{r}\left(k_{\perp}\right) \delta\left(k_{\perp}-k_{\perp}^{\prime}\right) \equiv N_{m}\left(k_{\perp}\right) \delta\left(k_{\perp}-k_{\perp}^{\prime}\right),
\end{aligned}
$$

and similarly for TE polarization, such that

$$
\begin{gathered}
N_{\phi, m}= \begin{cases}2 \pi & m=0 \\
\pi & m \neq 0\end{cases} \\
N_{r}\left(k_{\perp}\right) \delta\left(k_{\perp}-k_{\perp}^{\prime}\right)=\int_{0}^{\infty} J_{m}\left(k_{\perp} r\right) J_{m}\left(k_{\perp}^{\prime} r\right) r d r=\frac{\delta\left(k_{\perp}-k_{\perp}^{\prime}\right)}{k_{\perp}} .
\end{gathered}
$$

Our basis modes are then given by

$$
\begin{aligned}
& \mathbf{f}_{\mathrm{TM}}\left(k_{\perp}, m, r, \phi\right)=\left[\begin{array}{ll}
f_{r, \mathrm{TM}}\left(k_{\perp}, m, r, \phi\right) & f_{\phi, \mathrm{TM}}\left(k_{\perp}, m, r, \phi\right)
\end{array}\right]^{T} \\
& =\frac{1}{\sqrt{N_{m}\left(k_{\perp}\right)}}\left[\begin{array}{ll}
\frac{1}{k_{\perp}} \frac{\partial J_{m}\left(k_{\perp} r\right)}{\partial r} \cos (m \phi) & -\frac{m}{k_{\perp} r} J_{m}\left(k_{\perp} r\right) \sin (m \phi)
\end{array}\right]^{T} \\
& \mathbf{g}_{\mathrm{TM}}\left(k_{\perp}, m, r, \phi\right)=\left[\begin{array}{ll}
g_{r, \mathrm{TM}}\left(k_{\perp}, m, r, \phi\right) & g_{\phi, \mathrm{TM}}\left(k_{\perp}, m, r, \phi\right)
\end{array}\right]^{T} \\
& =\frac{1}{\sqrt{N_{m}\left(k_{\perp}\right)}}\left[\begin{array}{ll}
\frac{m}{k_{\perp} r} J_{m}\left(k_{\perp} r\right) \sin (m \phi) & \frac{1}{k_{\perp}} \frac{\partial J_{m}\left(k_{\perp} r\right)}{\partial r} \cos (m \phi)
\end{array}\right]^{T} \\
& \mathbf{f}_{\mathrm{TE}}\left(k_{\perp}, m, r, \phi\right)=\left[\begin{array}{ll}
f_{r, \mathrm{TE}}\left(k_{\perp}, m, r, \phi\right) & f_{\phi, \mathrm{TE}}\left(k_{\perp}, m, r, \phi\right)
\end{array}\right]^{T} \\
& =\frac{1}{\sqrt{N_{m}\left(k_{\perp}\right)}}\left[\begin{array}{ll}
\frac{1}{k_{\perp}} \frac{\partial J_{m}\left(k_{\perp} r\right)}{\partial r} \sin (m \phi) & \frac{m}{k_{\perp} r} J_{m}\left(k_{\perp} r\right) \cos (m \phi)
\end{array}\right]^{T}
\end{aligned}
$$




$$
\begin{aligned}
& \mathbf{g}_{\mathrm{TE}}\left(k_{\perp}, m, r, \phi\right)=\left[g_{r, \mathrm{TE}}\left(k_{\perp}, m, r, \phi\right) \quad g_{\phi, \mathrm{TE}}\left(k_{\perp}, m, r, \phi\right)\right]^{T} \\
& =\frac{1}{\sqrt{N_{m}\left(k_{\perp}\right)}}\left[\frac{m}{k_{\perp} r} J_{m}\left(k_{\perp} r\right) \cos (m \phi) \quad-\frac{1}{k_{\perp}} \frac{\partial J_{m}\left(k_{\perp} r\right)}{\partial r} \sin (m \phi)\right]^{T} \text {. }
\end{aligned}
$$

The product integral is

$$
\begin{aligned}
& \int_{u=u_{1}, v=0}^{u=u_{2}, v=2 \pi} U\left(k_{\perp}^{\prime}, m, u\right) V\left(k_{\perp}^{\prime}, m, v\right) U\left(k_{\perp}, m, u\right) V\left(k_{\perp}, m, v\right) l_{u} l_{v} d u d v \\
& =\int_{r=r_{1}, \phi=0}^{r=r_{2}, \phi=2 \pi} r J_{m}\left(k_{\perp}^{\prime} r\right) J_{m}\left(k_{\perp} r\right) \Phi_{m, \mathrm{TX}}^{2}(\phi) r d r d \phi
\end{aligned}
$$

where the angular function $\Phi_{m, \mathrm{TX}}(\phi)$ is given by either (234) or (235).

The radial part of the integration can be performed using the following indefinite integrals

$$
\begin{gathered}
\int J_{m}\left(k_{\perp}^{\prime} r\right) J_{m}\left(k_{\perp} r\right) r d r=r \frac{k_{\perp} J_{m-1}\left(k_{\perp} r\right) J_{m}\left(k_{\perp}^{\prime} r\right)-k_{\perp}^{\prime} J_{m-1}\left(k_{\perp}^{\prime} r\right) J_{m}\left(k_{\perp} r\right)}{k_{\perp}^{\prime 2}-k_{\perp}^{2}} \quad\left(k_{\perp}^{\prime} \neq k_{\perp}\right) \\
\int J_{m}\left(k_{\perp} r\right)^{2} r d r=\frac{r^{2}}{2}\left(J_{m}\left(k_{\perp} r\right)^{2}-J_{m-1}\left(k_{\perp} r\right) J_{m+1}\left(k_{\perp} r\right)\right) .
\end{gathered}
$$

\subsection{Boundary terms in integration by parts}

Finally, we note that

$$
\begin{gathered}
J_{m}(0)=0 \quad(m \neq 0) \\
\frac{\partial}{\partial r} J_{0}(0)=0,
\end{gathered}
$$

which leads to the cancellation of the $u=r=0$ contribution to the boundary term in Eq. (17). Additionally, we have

$$
U_{\mathrm{TX}}(0) \frac{\partial}{\partial v} V_{\mathrm{TX}}(v)=J_{m}(0) \frac{\partial}{\partial \phi} \cos (m \phi)=J_{m}(0) \frac{\partial}{\partial \phi} \sin (m \phi)=0
$$

for all values of $m$, such that the $u=r=0$ contribution to the boundary term in Eq. (18) disappears.

\section{Elliptical cylindrical coordinates}

The coordinate transformation for the open geometry elliptical coordinate system is given by

$$
\begin{aligned}
& x=f \cosh (u) \cos (v) \\
& y=f \sinh (u) \sin (v),
\end{aligned}
$$

with $0 \leq u \leq \infty$ for the open geometry and $0 \leq v \leq 2 \pi$ as before. The scale factors are

$$
l_{u}=l_{v}=f\left[\frac{1}{2}(\cosh 2 u-\cos 2 v)\right]^{\frac{1}{2}},
$$

where $f$ is the focal distance defined below. An ellipse is described by

$$
\left(\frac{x}{r_{x}}\right)^{2}+\left(\frac{y}{r_{y}}\right)^{2}=1
$$


which defines a line of constant $u$, where $u$ and $f$ are related the radii of the semi-major $r_{x}$ and semiminor $r_{y}$ axes as

$$
\begin{gathered}
\cosh (2 u)=\frac{r_{x}^{2}+r_{y}^{2}}{r_{x}^{2}-r_{y}^{2}} \\
f=\sqrt{r_{x}^{2}-r_{y}^{2}} .
\end{gathered}
$$

The Helmholtz equation (6) in elliptical coordinates for the empty geometry is

$$
\frac{2}{f^{2}(\cosh 2 u-\cos 2 v)}\left(\frac{\partial^{2}}{\partial u^{2}}+\frac{\partial^{2}}{\partial v^{2}}\right) E(u, v)+k_{0}^{2} E(u, v)=\beta^{2} E(u, v) \text {. }
$$

We then insert $E(u, v)=U(u) V(v)$ into Eq. (257) and divide by $U V$ to obtain

$$
\frac{2}{\cosh (2 u)-\cos (2 v)}\left(\frac{1}{U} \frac{\partial^{2} U}{\partial u^{2}}+\frac{1}{V} \frac{\partial^{2} V}{\partial v^{2}}\right)=-f^{2} k_{\perp}^{2},
$$

where $k_{\perp}^{2}=k_{0}^{2}-\beta^{2}$ as before. Rearranging Eq. (258) gives

$$
\left(\frac{1}{U} \frac{\partial^{2} U}{\partial u^{2}}+\frac{f^{2} k_{\perp}^{2}}{2} \cosh (2 u)\right)+\left(\frac{1}{V} \frac{\partial^{2} V}{\partial v^{2}}-\frac{f^{2} k_{\perp}^{2}}{2} \cos (2 v)\right)=0,
$$

and separation of the two terms gives

$$
\begin{gathered}
\frac{\partial^{2} V}{\partial v^{2}}+\left[a-\frac{f^{2} k_{\perp}^{2}}{2} \cos (2 v)\right] V=0 \\
\frac{\partial^{2} U}{\partial u^{2}}-\left[a-\frac{f^{2} k_{\perp}^{2}}{2} \cosh (2 u)\right] U=0,
\end{gathered}
$$

where $a$ is a separation constant. Eq. (260) is the Mathieu differential equation and Eq. (261) is the modified Mathieu differential equation. They define the radial and angular Mathieu functions $U(u)$ and $V(v)$, which are the equivalents of the Bessel and trigonometric functions for the elliptical coordinate system. Unlike the Bessel and trigonometric functions, most mathematical programming languages including MATLAB do not include built-in support for Mathieu functions, and instead they are computed as expansions on built-in functions.

Several procedures for solving the Mathieu differential equations have been suggested, and a useful MATLAB toolbox is also available [2]. We adopt the notation of Ref. [2] while modifying the normalization constants, with the help of the orthonormalization relation given in Ref. [3], to ensure that our normalization condition Eq. (135) is fulfilled.

The solutions to Eqs. (260)-(261) can be classified in four symmetry groups, even-even, even-odd, odd-odd, odd-even, according to their parity along the $y$ and $x$ axis respectively. The angular Mathieu functions can then be written as expansions on the trigonometric functions as

$$
\begin{gathered}
V_{\mathrm{ee}}\left(k_{\perp}, m, v\right)=\sum_{j=0}^{\infty} A_{\mathrm{ee}}^{(2 j)}\left(k_{\perp}, m\right) \cos (2 j v) \\
V_{\mathrm{eo}}\left(k_{\perp}, m, v\right)=\sum_{j=0}^{\infty} A_{\mathrm{eo}}^{(2 j+1)}\left(k_{\perp}, m\right) \cos [(2 j+1) v] \\
V_{\mathrm{oo}}\left(k_{\perp}, m, v\right)=\sum_{j=1}^{\infty} A_{\mathrm{oo}}^{(2 j)}\left(k_{\perp}, m\right) \sin (2 j v) \\
V_{\mathrm{oe}}\left(k_{\perp}, m, v\right)=\sum_{j=0}^{\infty} A_{\mathrm{oe}}^{(2 j+1)}\left(k_{\perp}, m\right) \sin [(2 j+1) v]
\end{gathered}
$$


where $A_{\mathrm{xy}}^{(j)}$ represents expansion coefficients with the subindex e (o) denoting even (odd) parity and $m$ denotes the angular order.

The $A$ coefficients are calculated by inserting the expansions into the differential equation and solving an eigenvalue problem $[2,4]$ and the expansion coefficients are normalized to ensure that

$$
\int_{0}^{2 \pi} V_{\mathrm{xy}}^{2}\left(k_{\perp}, m, v\right)=1
$$

The radial Mathieu function of the first kind can be written using Bessel functions as

$$
\begin{gathered}
U_{\mathrm{ee}}\left(k_{\perp}, m, u\right)=\frac{\sqrt{k_{\perp}}}{A_{\mathrm{ee}}^{(0)}} \sum_{j=0}^{\infty}(-1)^{j} A_{\mathrm{ee}}^{(2 j)}\left(k_{\perp}, m\right) J_{j}\left(p_{1}\right) J_{j}\left(p_{2}\right) \\
U_{\mathrm{eo}}\left(k_{\perp}, m, u\right)=\frac{\sqrt{k_{\perp}}}{A_{\mathrm{eo}}^{(1)}} \sum_{j=0}^{\infty}(-1)^{j} A_{\mathrm{eo}}^{(2 j+1)}\left(k_{\perp}, m\right)\left[J_{j}\left(p_{1}\right) J_{j+1}\left(p_{2}\right)+J_{j}\left(p_{2}\right) J_{j+1}\left(p_{1}\right)\right] \\
U_{\mathrm{oo}}\left(k_{\perp}, m, u\right)=\frac{\sqrt{k_{\perp}}}{A_{\mathrm{oo}}^{(2)}} \sum_{j=1}^{\infty}(-1)^{j} A_{\mathrm{oo}}^{(2 j)}\left(k_{\perp}, m\right)\left[J_{j-1}\left(p_{1}\right) J_{j+1}\left(p_{2}\right)-J_{j-1}\left(p_{2}\right) J_{j+1}\left(p_{1}\right)\right] \\
U_{\mathrm{oe}}\left(k_{\perp}, m, u\right)=\frac{\sqrt{k_{\perp}}}{A_{\mathrm{oe}}^{(1)}} \sum_{j=0}^{\infty}(-1)^{j} A_{\mathrm{oe}}^{(2 j+1)}\left(k_{\perp}, m\right)\left[J_{j}\left(p_{1}\right) J_{j+1}\left(p_{2}\right)-J_{j}\left(p_{2}\right) J_{j+1}\left(p_{1}\right)\right]
\end{gathered}
$$

where $p_{1}=\left(k_{\perp} f / 2\right) \exp (-u)$ and $p_{2}=\left(k_{\perp} f / 2\right) \exp (u)$. Note that the expansion coefficients are the same as for the angular and radial Mathieu functions. The integral $\int_{0}^{2 \pi} V_{\mathrm{xy}}(v) V_{\mathrm{x}^{\prime} \mathrm{y}^{\prime}}(v) d v$ leads to a decoupling of the four symmetry groups, and we may chose limit the calculation to include only even-odd modes for the TM basis set and odd-even modes for the TE basis such that

$$
\begin{aligned}
& \Psi_{\mathrm{TM}}\left(k_{\perp}, m, u, v\right)=U_{\text {eо }}\left(k_{\perp}, m, u\right) V_{\mathrm{eo}}\left(k_{\perp}, m, v\right) \\
& \Psi_{\mathrm{TE}}\left(k_{\perp}, m, u, v\right)=U_{\mathrm{oe}}\left(k_{\perp}, m, u\right) V_{\mathrm{oe}}\left(k_{\perp}, m, v\right) .
\end{aligned}
$$

Consequently, the basis modes can be written as

$$
\begin{aligned}
& \mathbf{f}_{\mathrm{TM}}\left(k_{\perp}, m, u, v\right)=\left[f_{u, m, \mathrm{TM}}\left(k_{\perp}, m, u, v\right) \quad f_{v, m, \mathrm{TM}}\left(k_{\perp}, m, u, v\right)\right]^{T} \\
& =\left[\frac{1}{k_{\perp} l_{u}} \frac{\partial U_{\mathrm{eo}}\left(k_{\perp}, m, u\right)}{\partial u} V_{\mathrm{eo}}\left(k_{\perp}, m, v\right) \frac{1}{k_{\perp} l_{v}} U_{\mathrm{eo}}\left(k_{\perp}, m, u\right) \frac{\partial V_{\mathrm{eo}}\left(k_{\perp}, m, v\right)}{\partial v}\right]^{T} \\
& \mathbf{g}_{\mathrm{TM}}\left(k_{\perp}, m, u, v\right)=\left[g_{u, \mathrm{TM}}\left(k_{\perp}, m, u, v\right) \quad g_{v, \mathrm{TM}}\left(k_{\perp}, m, u, v\right)\right]^{T} \\
& =\left[-\frac{1}{k_{\perp} l_{v}} U_{\mathrm{eo}}\left(k_{\perp}, m, u\right) \frac{\partial V_{\mathrm{eo}}\left(k_{\perp}, m, v\right)}{\partial v} \quad \frac{1}{k_{\perp} l_{u}} \frac{\partial U_{\mathrm{eo}}\left(k_{\perp}, m, u\right)}{\partial u} V_{\mathrm{eo}}\left(k_{\perp}, m, v\right)\right]^{T} \\
& \mathbf{f}_{\mathrm{TE}}\left(k_{\perp}, m, u, v\right)=\left[f_{u, m, \mathrm{TE}}\left(k_{\perp}, m, u, v\right) \quad f_{v, m, \mathrm{TE}}\left(k_{\perp}, m, u, v\right)\right]^{T} \\
& =\left[\frac{1}{k_{\perp} l_{u}} \frac{\partial U_{\mathrm{oe}}\left(k_{\perp}, m, u\right)}{\partial u} V_{\mathrm{oe}}\left(k_{\perp}, m, v\right) \frac{1}{k_{\perp} l_{v}} U_{\mathrm{oe}}\left(k_{\perp}, m, u\right) \frac{\partial V_{\mathrm{oe}}\left(k_{\perp}, m, v\right)}{\partial v}\right]^{T} \\
& \mathbf{g}_{\mathrm{TE}}\left(k_{\perp}, m, u, v\right)=\left[g_{u, \mathrm{TE}}\left(k_{\perp}, m, u, v\right) \quad g_{v, \mathrm{TE}}\left(k_{\perp}, m, u, v\right)\right]^{T} \\
& =\left[\frac{1}{k_{\perp} l_{v}} U_{\mathrm{oe}}\left(k_{\perp}, m, u\right) \frac{\partial V_{\mathrm{oe}}\left(k_{\perp}, m, v\right)}{\partial v}-\frac{1}{k_{\perp} l_{u}} \frac{\partial U_{\mathrm{oe}}\left(k_{\perp}, m, u\right)}{\partial u} V_{\mathrm{oe}}\left(k_{\perp}, m, v\right)\right]^{T} .
\end{aligned}
$$

The representation Eqs. (273)-(276) represents the eo-oe class for TM and TE modes, respectively, which includes the fundamental $x$-polarized $\mathrm{HE}_{11}$ mode. To take into account all of the possible solutions, basis sets formed from the other three symmetry groups (oe-eo, ee-oo and oo-ee) should also be considered. Unlike the rotationally symmetric structure, the angular Mathieu functions with different angular order $m$ are not orthogonal, and in principle all angular orders $m$ should be 
included in a calculation. However, as discussed in the main article Section 3C, only a few orders are typically needed for convergence.

\subsection{Boundary terms in integration by parts}

We note that the radial Mathieu functions $U_{\mathrm{xy}}\left(k_{\perp}, m, u\right)$ are either even or odd functions with respect to $u$, and thus either the function itself or its derivative is zero at $u=0$ such that

$$
U_{\mathrm{xy}}\left(k_{\perp}, m, 0\right) \frac{\partial}{\partial u} U_{\mathrm{xy}}\left(k_{\perp}, m, 0\right)=0
$$

for all four symmetry groups ensuring the cancellation of the $u=0$ contribution to the boundary term in Eq. (17).

Finally we note that the radial Mathieu functions $U_{\mathrm{TX}}(u)$ and $U_{\mathrm{TY}}(u)$ belong to different even-odd symmetry classes as defined in Eqs. (271)-(272). Either $U_{\mathrm{TX}}(0)$ or $U_{\mathrm{TY}}(0)$ will thus be zero such that

$$
U_{q^{\prime}, \mathrm{TX}}(0) U_{q, \mathrm{TY}}(0)=0,
$$

leading to the cancellation of the $u=0$ contribution to the boundary term in Eq. (18).

\section{References}

1. A. V. Lavrinenko, J. Lægsgaard, N. Gregersen, F. Schmidt, and T. Søndergaard, Numerical Methods in Photonics (CRC Press, 2015).

2. E. Cojocaru, "Mathieu functions computational toolbox implemented in Matlab," arXiv 0811.1970 (2008).

3. A. A. Inayat-Hussain, "Mathieu integral transforms," J. Math. Phys. 32, 669-675 (1991).

4. N. W. McLachlan, Theory and Application of Mathieu Functions (Oxford University Press, 1964). 